NASA-CR-199044

$$
\begin{gathered}
\text { 191- } 30 \\
11-61- \pm 2 \\
60=\times 5
\end{gathered}
$$

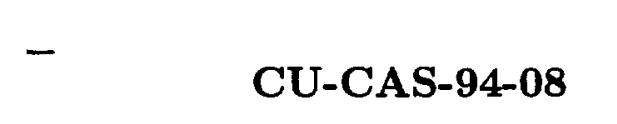

CENTER FOR AEROSPACE STRUCTURES

p. 58

\title{
A SURVEY OF THE CORE- CONGRUENTIAL FORMULATION FOR GEOMETRICALLY NONLINEAR TL FINITE ELEMENTS
}

(NASA-CR-199044) A SURVEY OF THE CORE-CONGRUENTIAL FORMULATION FOR GFOMETRICALLY NCNLINEAR TL FINITE ELEMENTS (Colorado Univ.) $58 \mathrm{p}$ Unclas N95-32203

$63 / 610060385$

by

C. A. Felippa, L. A. Crivelli and $B$. Haugen

May 1994

COLLEGE OF ENGINEERING UNIVERSITY OF COLORADO CAMPUS BOX 429 BOULDER, COLORADO 80309 


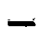

$-$

$-$

-

$-$

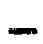

$-$

$-$

$-$

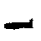

$-$

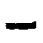

$\rightarrow$ 


\title{
A Survey of the Core-Congruential Formulation for Geometrically Nonlinear TL Finite Elements
}

\author{
Carlos A. Felippa, luis A. Crivelli \\ AND BJøRN HAUGEN \\ Department of Aerospace Engineering Sciences \\ and Center for Aerospace Structures \\ University of Colorado \\ Boulder, Colorado 80309-0429, USA
}

May 1994

Report No. CU-CAS-94-08

\begin{abstract}
Invited survey article contributed to the inaugural issue of Archives of Computational Methods in Engineering. Editors: M. Kleiber, E. Oñate and O. C. Zienkiewicz. To appear August 1994.

The research surveyed in this article was supported by the Air Force Office of Scientific Research under Grant F49620-87-C-0074, NASA Langley Research Center under Grant NAG1-756, NASA Lewis Research Center under Grant NAG3-1273, and the National Science Foundation under Grant ASC-9217394.
\end{abstract}




\title{
A Survey of the Core-Congruential Formulation for Geometrically Nonlinear TL Finite Elements
}

\author{
C. A. FELIPPA*, L. A. CRIVELLI ${ }^{* *}$ and B. HAUGEN ${ }^{* * *}$
}

\section{SUMMARY}

This article presents a survey of the Core-Congruential Formulation (CCF) for geometrically nonlinear mechanical finite elements based on the Total Lagrangian (TL) kinematic description. Although the key ideas behind the CCF can be traced back to Rajasekaran and Murray in 1973, it has not subsequently received serious attention. The CCF is distinguished by a two-phase development of the finite element stiffness equations. The initial phase develop equations for individual particles. These equations are expressed in terms of displacement gradients as degrees of freedom. The second phase involves congruential-type transformations that eventually binds the element particles of an individual element in terms of its node-displacement degrees of freedom. Two versions of the CCF, labeled Direct and Generalized, are distinguished. The Direct CCF (DCCF) is first described in general form and then applied to the derivation of geometrically nonlinear bar, and plane stress elements using the Green-Lagrange strain measure. The more complex Generalized CCF (GCCF) is described and applied to the derivation of $2 \mathrm{D}$ and 3D Timoshenko beam elements. Several advantages of the CCF, notably the physically clean separation of material and geometric stiffnesses, and its independence with respect to the ultimate choice of shape functions and element degrees of freedom, are noted. Application examples involving very large motions solved with the 3D beam element display the range of applicability of this formulation, which transcends the kinematic limitations commonly attributed to the TL description.

\section{INTRODUCTION}

There is an elegant Total Lagrangian (TL) formulation of geometrically nonlinear mechanical finite elements that has received little attention in the literature. This will be referred to as the Core-Congruential Formulation, or CCF, in the sequel. The key concepts, presented by Rajasekaran and Murray ${ }^{1}$ in 1973 , evolved from the analysis and reinterpretation of the pioneer work of Mallet and Marcal, ${ }^{2}$ as well as Murray's previous work in geometrically nonlinear finite element analysis. ${ }^{3}$ The discussion of Reference ${ }^{1}$ by Felippa ${ }^{4}$ provided parametric expressions for the stiffness matrices that appear at various levels of the discrete governing equations. This work originated what is called here the Direct Core Congruential Formulation, or DCCF.

In 1987 a course in nonlinear finite elements offered by the first author presented the derivation of several elements using the DCCF. Preparation of homework assignments and

- Department of Aerospace Engineering Sciences and Center for Aerospace Structures, University of Colorado, Boulder, CO 80309-0429, USA

** Hibbit, Karlsson \& Sorensen Associates, 1080 Main St., Pawtucket, RI 02860, USA

*** Division of Structural Engineering, The Norwegian Institute of Technology, N-7034 Trondheim, Norway 
feedback from students in this and follow-up offerings helped to streamline the material. Subsequently Crivelli's doctoral thesis ${ }^{5}$ used the CCF in the systematic development of a three-dimensional nonlinear Timoshenko beam element capable of undergoing arbitrarily large rotations. Challenges posed by this application pushed this formulation beyond frontiers hitherto deemed impassable by a TL element with rotational degrees of freedom. This development was summarily reported in a survey article by Felippa and Crivelli ${ }^{6}$ and explained in more detail in a subsequent paper by Crivelli and Felippa. ${ }^{7}$

A lesson gained from this research is that, when dealing with $3 \mathrm{D}$ finite rotations, the $\mathrm{CCF}$ should be applied in a staged fashion that allows the systematic examination of additional terms arising in the transformations to physical degrees of freedom. That transformation methodology gave rise to what is here called the Generalized CCF, or GCCF.

Both DCCF and GCCF share the same "divide and conquer" philosophy. However, the core equations as well as subsequent steps that transform those equations to physical freedoms vary in complexity. To simplify the exposition while focusing on the essential aspects, Sections 3 through 7 focus on the DCCF. Examples of application to elements amenable to the direct treatment are presented. The GCCF is discussed in Sections 8 through 10 , and illustrated with applications to $2 \mathrm{D}$ and 3D beam elements.

REMARK 1.1. Several authors have expressed the belief that the approximation performance of TL-based elements degrades beyond moderate rotations, and an updated Lagrangian or corotational description is necessary for handling truly large motions. For example, in 1986 Mathiasson, Bengtsson and Samuelsson ${ }^{8}$ concluded that "The TL formulation can only be used in problems with small or moderate displacements." More recently Bergan and Mathisen ${ }^{9}$ voice a similar opinion: "it is commonly known that in a step by step TL formulation artificial strains easily arise in beam elements due to nonhomogeneities in the displacement expansions in transverse and longitudinal directions." Our experience shows that such limitations are not inherent in the TL description but instead emerge when a priori kinematic approximations are made to simplify element derivations. The $3 \mathrm{D}$ beam element just cited exhibits computational and approximation performance for very large rotations comparable to those based on the co-rotational and Updated Lagrangian descriptions while retaining certain advantages listed in the Conclusions.

\section{OVERVIEW}

\subsection{Basic Concepts}

The original development of the CCF was concerned with the construction of TL stiffness matrices for geometrically nonlinear analysis through the congruential-transformation pattern

$$
\mathbf{K}^{l e v e l}=\int_{V_{0}} \mathbf{G}^{T} \mathbf{S}^{l e v e l} \mathbf{G} d V,
$$

where $\mathbf{S}$ is the core stiffness matrix, $\mathbf{K}$ the physical stiffness in terms of the nodal degrees of freedom $\mathbf{v}, \mathbf{G}$ a core-to-physical-freedom transformation matrix assumed to be independent of $\mathbf{v}, V_{0}$ the appropriate reference integration volume, and in which "level" identifies the governing equation level at which the stiffness matrix is used. 
The three variational levels of interest in practice are: energy (level 0 ), force equilibrium (level 1), and first-order incremental equilibrium (level 2). Qualifiers "residual-force" and "secant-stiffness" are also used for level 1, and "tangent-stiffness" used for level 2.

The core stiffness matrix is expressed in terms of the displacement gradients at each material point. Displacement gradients $\mathbf{g}$ make a better choice of core variables than finite strains because for elements with translational degrees of freedom (DOFs) they can be expressed linearly in terms of node displacements $\mathbf{v}$ as $\mathbf{g}=\mathbf{G v}$, a property that validates (2.1) for all levels. As discussed below, such elements fall under the purview of the Direct CCF.

The qualifier "core" emphasizes the goal of independence of $\mathrm{S}^{\text {level }}$ with respect to discretization decisions such as element geometry, shape functions, and choice of nodal degrees of freedom. Such a dependence is introduced by the congruential transformation indicated in (2.1) and the integration over the element volume.

\subsection{Direct and Generalized CCF}

The basic schematics of the CCF, mathematically expressed through (2.1), may be diagrammed as

\begin{tabular}{|c|c|c|c|c|}
\hline $\begin{array}{l}\text { Core } \\
\text { Stiffness } \\
\text { Equations }\end{array}$ & $\Longrightarrow$ & $\begin{array}{c}\text { Congruential } \\
\text { Transformation } \\
\text { Equations }\end{array}$ & $\Longrightarrow$ & $\begin{array}{c}\text { Physical-DOF } \\
\text { Stiffness } \\
\text { Equations }\end{array}$ \\
\hline
\end{tabular}

But this panoramic view needs to be rendered more precise. If the relation between core DOFs (the displacement gradients $\mathbf{g}$ ) and the physical DOFs (the node displacements $\mathbf{v}$ of a finite element model) is linear, these transformations do not depend on level:

$\left.\begin{array}{|c|c|}\hline \begin{array}{c}\text { (0) Core Energy Stiffness } \\
\text { (1) Core Secant Stiffness } \\
\text { (1) Core Internal Force }\end{array}\end{array} \Longrightarrow \begin{array}{c}\begin{array}{c}\text { Congruential } \\
\text { Transformation } \\
\text { Equations }\end{array} \\
\text { (2) Tangent Stiffness }\end{array}\right]$\begin{tabular}{c}
$\begin{array}{c}\text { Physical Energy Stiffness } \\
\text { Physical Secant Stiffness } \\
\text { Physical Internal Force } \\
\text { Physical Tangent Stiffness }\end{array}$ \\
\hline
\end{tabular}

In this diagram, numbers annotated within the "core box" denote the variational level of the governing equation in use. Internal force and secant stiffness are two alternative governing-equation expressions at level 1. The energy level (level 0 ) may also be expressed in several ways, but this is not shown in the diagrams to reduce clutter. Under the aforementioned assumption we obtain the Direct Core Congruential Formulation, or DCCF. If the relation between displacement gradients $\mathbf{g}$ and node displacements $\mathbf{v}$ is nonlinear, the transformations sketched above are not only more complex but depend on variational 
level and possibly the expression form used within a level. This complication arises when elements with rotational degrees of freedom such as beams, plates and shells are considered. It gives rise to the Generalized Core Congruential Formulation, or GCCF.

Two variants of the GCCF may be distinguished. If the relation between $\mathbf{g}$ and $\mathbf{v}$ is nonlinear but algebraic, the transformation equations do vary with level but in principle are still possible as illustrated in the following diagram.

\begin{tabular}{l}
\begin{tabular}{|c|c|}
\hline $\begin{array}{c}\text { (0) Core Energy Stiffness } \\
\text { (1) Core Secant Stiffness }\end{array}$ & $\Rightarrow \begin{array}{c}\text { S-Congruential } \\
\text { Transformation } \\
\text { Equations }\end{array}$ \\
\begin{tabular}{|c|}
\hline (1) Core Internal Force \\
(2) Core Tangent Stiffness
\end{tabular}
\end{tabular}$\Longrightarrow$\begin{tabular}{|c|} 
T-Congruential \\
Transformation \\
Equations
\end{tabular} \\
\hline
\end{tabular}

Here "T-Congruential" and "S-Congruential" are abbreviations for "Tangent Congruential" and "Secant-Congruential," respectively. Such a distinction is elaborated upon in Section 8 .

If the relation between $\mathbf{g}$ and $\mathbf{v}$ is nonlinear and can be expressed only in non-integrable differential form, the "Secant Transformation Equations" of the preceding diagram do not generally exist, and the diagram must be truncated:

(0) Core Energy Stiffness

(1) Core Secant Stiffness

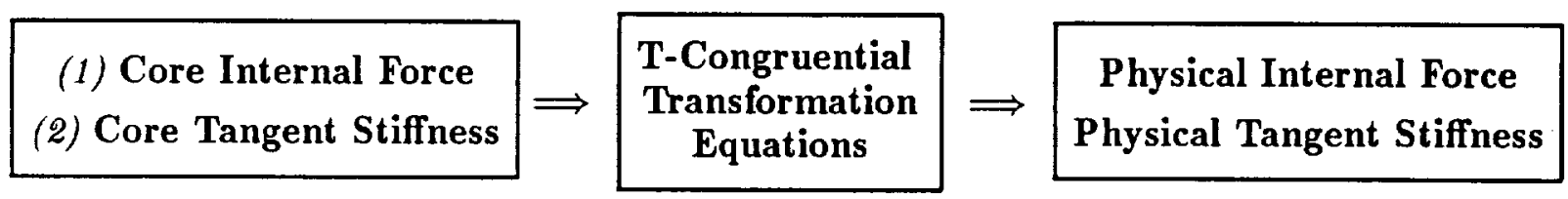

These two variants of the GCCF are called Algebraic GCCF and Differential GCCF and denoted by acronyms AGCCF and DGCCF, respectively, in the sequel. The main distinction between AGCCF and DGCCF is that it makes no sense to talk about missing quantities, such as the physical secant stiffness, with the latter.

The original development of the CCF outlined in the Introduction focused on elements with translational-degree-of-freedom configurations. For such elements the Direct form of the CCF, or DCCF, is sufficient. Sections 3 through focus on that form, leaving the development and application of the GCCF to Section 8 and following ones. 


\subsection{The CCF Philosophy: Divide and Conquer}

The CCF derivation of the finite element equations naturally reflects the outlined framework. It proceeds through two phases: a core phase followed by a transformation phase. In the initial phase core energy, secant and tangent stiffness matrices as well as internal force vectors are obtained. These matrices and vectors pertain to individual particles. For the stiffness matrices they are collectively represented by the term $S^{\text {level }}$ in (2.1).

The key goal is to try to make such core equations as independent as possible with respect to finite-element discretization decisions such as element geometry, shape functions, selection of nodal degrees of freedom and (in the case of rotational DOFs) rotational parametrizations. To emphasize this independence, the term core was coined. Complete independence is in fact achievable if the relation between displacement gradients $\mathbf{g}$ and $\mathbf{v}$ is linear, which characterizes the DCCF. The goal has to be tempered if the relation is nonlinear because dependencies may arise at the tangent stiffness level. Such dependencies create the so-called complementary geometric stiffness terms, which are characteristic of elements that fall under purview of the GCCF.

In the transformation phase, these core forms are transformed to physical DOFs, i.e. element node displacements. The transformation may be done directly for simple elements and in multistage fashion for complex ones. In particular, multistage transformations are recommended for elements that require the Differential GCCF such as 3D beam and shell elements. In this case the transformation phase is decomposed into transformation stages that progressively "bind" particles into lines, areas or volumes through kinematic constraints, and eventually link the element domain to the nodal degrees of freedom. Decisions such as the choice of specific parametrizations for finite rotations may be deferred to final stages.

What are the differences between the CCF and the more conventional Total Lagrangian formulation of nonlinear finite elements? If kinematic exactness is maintained throughout, the final discrete equations are identical. This is shown in Appendix 1 for the DCCF applied to continuum elements. But in geometrically nonlinear analysis approximations of various kinds are common, especially in structural elements with rotational degrees of freedom such as beams, plates and shells. In the conventional formulation it is quite difficult to assess a priori the effect of seemingly innocuous approximations "thrown into the pot," and a posteriori exhaustive testing of complex situations becomes virtually impossible. Sample: how does the neglect of higher order terms in the axial deformation of a spinning 3D beam affects torsional buckling?

The staged approach recommended for the GCCF permits a better control over such assumptions. The core equations are physically transparent, clearly displaying the effect of material behavior, displacement gradients and prestresses. In the ensuing transformation sequence the origin of each term can be accurately traced, and on that basis informed decisions on retention or dropping made. This process can be aided by computer by testing subproblems that isolate the physics modeled by specific terms. 
From this discussion it follows that, from the standpoint of element development, evaluation and testing, the most significant advantage that can be claimed for the CCF is the clean separation of physical effects. The importance of this factor should not be underestimated, because physical transparency is the key to success in nonlinear analysis.

\section{HISTORICAL BACKGROUND}

In 1968 Mallet and Marcal $^{2}$ attempted to establish a standard nomenclature for geometrically nonlinear finite element structural analysis based on the Total Lagrangian (TL) kinematic description. Consider a discrete, finite element model of a static structural system under dead loading with nodal displacement degrees of freedom collected in array v. Displacements are measured from a fixed reference configuration $\mathcal{C}_{0}$ to a current configuration $\mathcal{C}$. The virtual-work conjugate forces, independent of $\mathbf{v}$, are collected in array p. The system has a total potential energy function $J=U-W$ that is the difference between the strain energy $U$ and the loads potential $W=\mathbf{p}^{T} \mathbf{v}$. The residual node forces are $\mathbf{r}=\partial J / \partial \mathbf{v}$, and the symbol $\Delta$ denotes increment associated with the variation of the current configuration. (In keeping up with the spirit of Reference ${ }^{2}$ actual variations are used in this Section rather than virtual ones; the latter are identified by the usual $\delta$ prefix.)

Mallet and Marcal expressed the total potential energy, the residual (force-balance) equilibrium equations, and the incremental equilibrium equations as follows:

$$
\begin{aligned}
J=U-W & =\frac{1}{2} \mathbf{v}^{T}\left[\mathbf{K}_{0}+\frac{1}{3} \mathbf{N}_{1}+\frac{1}{6} \mathbf{N}_{2}\right] \mathbf{v}-\mathbf{p}^{T} \mathbf{v} \\
\mathbf{r}=\frac{\partial J}{\partial \mathbf{v}} & =\left[\mathbf{K}_{0}+\frac{1}{2} \mathbf{N}_{1}+\frac{1}{3} \mathbf{N}_{2}\right] \mathbf{v}-\mathbf{p}=\mathbf{0}, \\
\Delta \mathbf{r} & =\left[\mathbf{K}_{0}+\mathbf{N}_{1}+\mathbf{N}_{2}\right] \Delta \mathbf{v}-\Delta \mathbf{p}=\mathbf{0} .
\end{aligned}
$$

Here $\mathbf{K}_{0}$ is the linear stiffness matrix evaluated at the reference configuration, whereas $\mathrm{N}_{1}$ and $\mathrm{N}_{2}$ are nonlinear stiffness matrices, also evaluated at the reference configuration, that depend linearly and quadratically, respectively, on the node displacements $\mathbf{v}$. The $\mathbf{N}$ matrices were said "to repeat" in the foregoing expressions. (This old notation has not survived; presently symbol $\mathbf{N}$ is most commonly used to identify matrices of element shape functions.)

Five years later Rajasekaran and Murray ${ }^{1}$ examined more critically the structure of the matrices that appear in the above equations. In that investigation they chose to start from the "core" stiffness matrices corresponding to $K, N_{1}$ and $N_{2}$ expressed in terms of displacement gradients, and in doing so laid down the main idea of the CCF. Working with specific elements they showed that the nonlinear stiffness matrices $N_{1}$ and $N_{2}$ are not uniquely determined. Indeed (3.1)-(3.3) as written are unique only for a single degree of freedom. They did not present, however, a general expression valid for arbitrary elements. This was partly done by Felippa, ${ }^{4}$ who in the discussion of Reference ${ }^{1}$ considered again those equations, rewritten here in a more general and compact form:

$$
J=\frac{1}{2} \mathbf{v}^{T} \mathbf{K}^{U} \mathbf{v}+\left(\mathbf{p}^{0}-\mathbf{p}\right)^{T} \mathbf{v},
$$




$$
\begin{aligned}
\mathbf{r} & =\mathbf{K}^{r} \mathbf{v}+\mathbf{p}^{0}-\mathbf{p}=\mathbf{f}-\mathbf{p}=\mathbf{0} \\
\Delta \mathbf{r} & =\mathbf{K} \Delta \mathbf{v}-\Delta \mathbf{p}=\mathbf{0}
\end{aligned}
$$

in which the notation of this paper - rather than that of Reference ${ }^{4}$ is used. Here $\mathbf{K}^{U}$, $\mathbf{K}^{r}$ and $\mathbf{K}$ denote the energy, secant and tangent stiffness matrices, respectively. (Energy and secant stiffnesses are not denoted by $\mathbf{K}^{e}$ and $\mathbf{K}^{s}$ because such symbols are used for other purposes in the finite element course noted in the Introduction.) In addition, $\mathbf{p}^{0}$ is the prestress force vector, which vanishes if the reference configuration is stress free and was omitted in that discussion, ${ }^{4}$ and $f=\mathbf{K}^{r} \mathbf{v}+\mathbf{p}^{0}$ is the internal force vector. The tangent stiffness is of course fundamental in incremental-iterative solution methods and stability analysis, while the secant stiffness (by itself or in the internal-force form $\mathbf{K}^{r} \mathbf{v}+\mathbf{p}^{0}$ ) is important in pseudo-force methods. The energy stiffness enjoys limited application per se but has theoretical importance as source for the other two.

In linear problems $\mathbf{K}^{U}=\mathbf{K}^{r}=\mathbf{K}=\mathbf{K}_{0}$ and the three stiffness matrices coalesce. But in nonlinear problems not only do the matrices differ but, as shown in the next section, $\mathbf{K}^{U}$ and $\mathbf{K}^{r}$ may involve arbitrary scalar coefficients. Such parametrized expressions were given by Felippa ${ }^{4}$ under the following restrictions:

(R1) $\mathbf{K}^{r}$ is symmetric.

(R2) The reference configuration is stress free.

(R3) The finite strain measure is quadratic in the displacement gradients.

(R4) The transformation between core and physical freedoms is linear.

The following treatment eliminates restrictions (R1) and (R2) altogether, and the other two selectively. It should be noted that restriction (R4) is the condition that, with present terminology, characterizes the DCCF.

\section{CORE STIFFNESS EQUATIONS}

\subsection{TL Description of Particle Motion}

A conservative, geometrically nonlinear structure under dead loading is viewed as a continuum undergoing finite displacements $\mathbf{u}$. These displacements are measured from a fixed reference configuration $\mathcal{C}_{0}$ to a variable current configuration $\mathcal{C}$. No discretization into finite elements is implied at this stage. We confine our attention to the case in which the material behavior stays within the linear elastic range, thus implying small deformational strains but arbitrarily large rotations. Corresponding points or particles in the reference and current configuration are referred to a fixed Cartesian coordinate system and have the coordinates $X_{i}$ and $x_{i}\left(i=1, \ldots n_{d}\right)$, respectively, where $n_{d}$ is the number of space dimensions. The displacement field components are $u_{i}=x_{i}-X_{i}$.

Let the state of strain at a particle in the current configuration be characterized by $n_{s}$ strains $e_{i}\left(i=1,2, \ldots n_{s}\right)$ collected in an array $\mathbf{e}$, and let the corresponding conjugate 
stresses be $s_{i}\left(i=1,2, \ldots n_{s}\right)$, collected in an array $\mathbf{s}$. Using the summation convention the elastic stress-strain relations are written

$$
s_{i}=s_{i}^{0}+E_{i j} e_{j}, \quad \text { with } \quad E_{i j}=E_{j i}, \quad \text { or } \quad \mathbf{s}=\mathbf{s}^{0}+\mathbf{E e}
$$

where $s_{i}^{0}$ are stresses in the reference configuration (stresses that remain if $e_{i}=0$, also called prestresses) and $E_{i j}$ are elastic moduli arranged as a $n_{s} \times n_{s}$ square array in the usual manner.

Let $\mathcal{J}, \mathcal{U}, \mathcal{W}, \mathbf{\Psi}, \mathbf{\Phi}$ and $\boldsymbol{\Upsilon}$ denote the analogues of $J, U, W, \mathbf{p}, \mathbf{f}$ and $\mathbf{r}$, respectively, at the particle level. (The first three acquire the meaning of energy densities, whereas $\Psi$ is a dead-loading body force density independent of $\mathbf{u}$.) The strain energy density can be expressed as

$$
\mathcal{U}=e_{i} s_{i}^{0}+\frac{1}{2} e_{i} E_{i j} e_{j}=\mathbf{e}^{T} \mathbf{s}^{0}+\frac{1}{2} \mathbf{e}^{T} \mathbf{E} \mathbf{e} .
$$

The total strain energy $U$ is obtained by integrating (4.2) over the structure volume: $U=\int_{V_{0}} \mathcal{U} d V$; the integration taking place - as can be expected in a TL description over the reference configuration geometry.

Next, introduce the $n_{g}$ displacement gradients $g_{m n}=\partial u_{m} / \partial X_{n}$. These are subsequently identified as $g_{i}\left(i=1,2, \ldots n_{g}\right)$ so they can be conveniently arranged in a one-dimensional array g. Following Rajasekaran and Murray ${ }^{1}$ and Felippa ${ }^{4}$ assume that the strains $e_{i}$ are linked to the displacement gradients through matrix relations of the form

$$
\mathbf{e}_{i}=\mathbf{h}_{\mathbf{i}}^{T} \mathbf{g}+\frac{1}{2} \mathbf{g}^{T} \mathbf{H}_{i} \mathbf{g}, \quad i=1,2, \ldots n_{\mathbf{s}}
$$

where $\mathbf{h}_{i}$ and $\mathbf{H}_{i}$ are arrays of dimension $n_{g} \times 1$ and $n_{g} \times n_{g}$, respectively, with $\mathbf{H}_{i}$ symmetric. In the original References ${ }^{1,4}$ it was assumed that $\mathbf{H}_{i}$ is independent of $g$, which is the case for the Green-Lagrange strain measure. This restriction, labeled (R3) in Section 3, will be enforced below except in Section 4.5.

\subsection{Energy Variations}

As noted previously, for deriving core equations we regard the displacement gradients $\mathbf{g}$ as degrees of freedom. On substituting (4.1) and (4.3) into (4.2) we obtain the "core counterparts" of (3.4)-(3.6), in which $\mathbf{v}$ has become $\mathbf{g}$ :

$$
\begin{gathered}
\mathcal{J}=\mathcal{U}-\mathcal{W}=\frac{1}{2} \mathbf{g}^{T} \mathbf{S}^{U} \mathbf{g}+\left(\Psi^{0}-\Psi\right)^{T} \mathbf{g} \\
\mathbf{\Upsilon}=\frac{\partial \mathcal{H}}{\partial \mathrm{g}}=\mathbf{S}^{r} \mathbf{g}+\Psi^{0}-\Psi=\mathbf{\Phi}-\Psi=0 \\
\Delta \mathbf{Y}=\mathbf{S} \Delta \mathrm{g}-\Delta \Psi=0
\end{gathered}
$$

Here $\mathbf{S}^{U}, \mathbf{S}^{r}$ and $\mathbf{S}$ denote the energy, secant and tangent core stiffness matrices, and $\mathbf{\Psi}^{\mathbf{0}}$, which is independent of $\mathbf{g}$, is the core counterpart of $\mathbf{p}^{0}$. 
With this notation the first and second variations of the strain energy density can be expressed as

$$
\begin{gathered}
\delta \mathcal{U}=\delta \mathbf{g}^{T}\left(\mathbf{S}^{U} \mathbf{g}+\Psi^{0}\right)+\frac{1}{2} \mathbf{g}^{T} \delta \mathbf{S}^{U} \mathbf{g}=\delta \mathbf{g}^{T}\left(\mathbf{S}^{r} \mathbf{g}+\mathbf{\Psi}^{0}\right)=\delta \mathbf{g}^{T} \mathbf{\Phi} \\
\delta^{2} \mathcal{U}=\delta \mathbf{g}^{T} \mathbf{S}^{r} \delta \mathbf{g}+\delta \mathbf{g}^{T} \delta \mathbf{S}^{r} \mathbf{g}+\left(\delta^{2} \mathbf{g}\right)^{T} \mathbf{\Phi}=\delta \mathbf{g}^{T} \mathbf{S} \delta \mathbf{g}+\left(\delta^{2} \mathbf{g}\right)^{T} \mathbf{\Phi}
\end{gathered}
$$

These variational equations implicitly determine $\mathbf{S}^{r}, \boldsymbol{\Phi}$ and $\mathbf{S}$ from $\mathbf{S}^{U}$ and $\boldsymbol{\Psi}^{0}$. If the linearity restriction (R4) holds, the term in $\delta^{2} \mathbf{g}$ drops out as explained in the Remark below, and

$$
\delta^{2} \mathcal{U}=\delta \mathbf{g}^{T} \mathbf{S} \delta \mathbf{g}
$$

REMARK 4.1. If $\mathbf{g}=\mathbf{G} \mathbf{v}$ with $\mathbf{G}$ independent of $\mathbf{v}, \delta^{2} \mathbf{g}=\mathbf{G} \delta^{2} \mathbf{v}=\mathbf{0}$ because $\mathbf{v}$ are independent variables. On the other hand, if displacement gradients are nonlinear functions of node displacements expressable as $g_{i}=g_{i}\left(v_{j}\right)$, then

$$
\delta g_{i}=\frac{\partial g_{i}}{\partial v_{j}} \delta v_{j}=G_{i j} \delta v_{j}, \quad \delta^{2} g_{i}=\frac{\partial^{2} g_{i}}{\partial v_{j} \partial v_{k}} \delta v_{j} \delta v_{k}+\frac{\partial g_{i}}{\partial v_{j}} \delta^{2} / v_{j}^{0}=F_{i j k} \delta v_{j} \delta v_{k} .
$$

Thus $\delta \mathbf{g}$ is still $\mathbf{G} \delta \mathbf{v}$ but $\delta^{2} \mathbf{g}=(\mathbf{F} \delta \mathbf{v}) \delta \mathbf{v}$, where $\mathbf{F}$ is a cubic array. The presence of the term $\delta^{2} \mathbf{g}$ is taken into account in the GCCF discussed in Sections 8-10.

\subsection{Parametrized Forms}

For convenience introduce the following $n_{g} \times n_{g}$ matrices (with summation convention on $i, j=1, \ldots n_{g}$ implied):

$$
\begin{aligned}
& \mathbf{S}_{0}=E_{i j} \mathbf{h}_{i} \mathbf{h}_{j}, \quad \mathbf{S}_{1}=E_{i j} \mathbf{h}_{i} \mathbf{g}^{T} \mathbf{H}_{j}, \quad \mathbf{S}_{1}^{*}=E_{i j}\left(\mathbf{h}_{i}^{T} \mathbf{g}\right) \mathbf{H}_{j}, \\
& \mathbf{S}_{2}=E_{i j} \mathbf{H}_{i} \mathbf{g g}^{T} \mathbf{H}_{j}, \quad \mathbf{S}_{2}^{*}=E_{i j}\left(\mathbf{g}^{T} \mathbf{H}_{i} \mathbf{g}\right) \mathbf{H}_{j},
\end{aligned}
$$

in which parentheses are used to emphasize the grouping of scalar quantities such as $\mathbf{g}^{T} \mathbf{H}_{\mathbf{i}} \mathbf{g}$. It may be then verified that, if assumptions (R3)-(R4) of Section 3 hold, the core stiffnesses and prestress vector in (4.6)-(4.8) possess the general form:

$$
\begin{aligned}
\mathbf{S}^{U}(\alpha, \beta) & =\mathbf{S}_{0}+\frac{1}{2} \alpha\left(\mathbf{S}_{1}+\mathbf{S}_{1}^{T}\right)+(1-\alpha) \mathbf{S}_{1}^{*}+\frac{1}{4} \beta \mathbf{S}_{2}+\frac{1}{4}(1-\beta) \mathbf{S}_{2}^{*}+s_{i}^{0} \mathbf{H}_{i} \\
& =\mathbf{S}_{0}+\frac{1}{2} \alpha\left(\mathbf{S}_{1}+\mathbf{S}_{1}^{T}\right)+\left(\frac{1}{2}-\alpha\right) \mathbf{S}_{1}^{*}+\frac{1}{4} \beta\left(\mathbf{S}_{2}-\mathbf{S}_{2}^{*}\right)+\frac{1}{2}\left(s_{i}^{0}+s_{i}\right) \mathbf{H}_{i}, \\
& =\mathbf{S}_{0}+\frac{1}{2} \alpha\left(\mathbf{S}_{1}+\mathbf{S}_{1}^{T}\right)-\alpha \mathbf{S}_{1}^{*}+\frac{1}{4} \beta \mathbf{S}_{2}-\frac{1}{4}(1+\beta) \mathbf{S}_{2}^{*}+s_{i} \mathbf{H}_{i}, \\
\mathbf{S}^{r}(\phi, \psi) & =\mathbf{S}_{0}+\frac{1}{2} \mathbf{S}_{1}+\phi \mathbf{S}_{1}^{T}+(1-\phi) \mathbf{S}_{1}^{*}+\frac{1}{4}(2-\psi) \mathbf{S}_{2}+\frac{1}{4} \psi \mathbf{S}_{2}^{*}+s_{i}^{0} \mathbf{H}_{i} \\
& =\mathbf{S}_{0}+\frac{1}{2} \mathbf{S}_{1}+\phi \mathbf{S}_{1}^{T}+\left(\frac{1}{2}-\phi\right) \mathbf{S}_{1}^{*}+\frac{1}{4}(2-\psi) \mathbf{S}_{2}+\frac{1}{4}(\psi-1) \mathbf{S}_{2}^{*}+\frac{1}{2}\left(s_{i}^{0}+s_{i}\right) \mathbf{H}_{i}, \\
& =\mathbf{S}_{0}+\frac{1}{2} \mathbf{S}_{1}+\phi \mathbf{S}_{1}^{T}-\phi \mathbf{S}_{1}^{*}+\frac{1}{4}(2-\psi)\left(\mathbf{S}_{2}-\mathbf{S}^{*}\right)+s_{i} \mathbf{H}_{i}, \\
\mathbf{S} & =\mathbf{S}_{0}+\mathbf{S}_{1}+\mathbf{S}_{1}^{T}+\mathbf{S}_{1}^{*}+\mathbf{S}_{2}+\frac{1}{2} \mathbf{S}_{2}^{*}+s_{i}^{0} \mathbf{H}_{i}=\mathbf{S}_{0}+\mathbf{S}_{1}+\mathbf{S}_{1}^{T}+\mathbf{S}_{2}+s_{i} \mathbf{H}_{i}, \\
\boldsymbol{\Psi}^{0} & =s_{i}^{0} \mathbf{h}_{i} .
\end{aligned}
$$


Here $\alpha, \beta, \phi$ and $\psi$ are arbitrary scalar coefficients in the sense that $\mathbf{g}^{T} \mathbf{S}^{U} \mathbf{g}$ and $\mathbf{S}^{r} \mathbf{g}$ are independent of them. In fact,

$$
\Phi=\mathbf{S}^{r} \mathbf{g}+\Psi^{0}=s_{i} \mathbf{b}_{i}
$$

where $b_{i}$ is defined in (4.18) below. The expressions (4.12) are more general than those originally given by Felippa ${ }^{4}$ because restrictions (R1)-(R2) noted in Section 3 are no longer enforced. Note that the secant core stiffness $S^{r}$ becomes symmetric if $\phi=1 / 2$.

The "repeatable forms" (3.1)-(3.3) of Mallet and Marcal are obtained if $\alpha=\beta=\psi=2 / 3$ and $\phi=1 / 2$, in which case the combinations $\mathbf{S}_{1}+\mathbf{S}_{1}^{T}+\mathbf{S}_{1}^{*}$ and $\mathbf{S}_{2}+\frac{1}{2} \mathbf{S}_{2}^{*}$ become the core counterparts of $N_{1}$ and $N_{2}$, respectively. But this observation has largely historical interest. More physically relevant are the following combinations:

$$
\begin{aligned}
& \mathbf{S}_{D}=\mathbf{S}_{1}+\mathbf{S}_{1}^{T}+\mathbf{S}_{2}, \quad \mathbf{S}_{M}=\mathbf{S}_{0}+\mathbf{S}_{D} \\
& \mathbf{S}_{G}=\mathbf{S}_{1}^{*}+\frac{1}{2} \mathbf{S}_{2}^{*}+s_{i}^{0} \mathbf{H}_{i}=s_{i} \mathbf{H}_{i} .
\end{aligned}
$$

These are the core versions of the initial-displacement, material and geometric stiffness, respectively. The core tangent stiffness is $\mathbf{S}=\mathbf{S}_{0}+\mathbf{S}_{D}+\mathbf{S}_{G}=\mathbf{S}_{M}+\mathbf{S}_{G}$.

If the Generalized CCF is required for downstream element development as explained in Section $8, \mathbf{S}_{G}=s_{i} \mathbf{H}_{i}$ is called the principal core geometric stiffness and is denoted by $\mathbf{S}_{G P}$. In this case the combination

$$
\mathbf{S}=\mathbf{S}_{M}+\mathbf{S}_{G P}
$$

receives the name principal core tangent stiffness.

REMARK 4.2. Finite element practicioners may be surprised at the nonuniqueness of $\mathbf{S}^{U}$ and $\mathbf{S}^{r}$. It appears to contradict the fact that, given two square matrices $\mathbf{A}_{1}$ and $\mathbf{A}_{2}$ and an arbitrary nonzero test vector $\mathbf{x}, \mathbf{A}_{1} \mathbf{x}=\mathbf{A}_{2} \mathbf{x}$ for all $\mathbf{x}$ implies $\mathbf{A}_{1}=\mathbf{A}_{2}$. But this is not necessarily true if $\mathbf{A}_{1}$ and $\mathbf{A}_{2}$ are functions of $\mathbf{x}$. More precisely, the energy core stiffness is not unique because

$$
\mathbf{g}^{T}\left(\mathbf{S}_{1}-\mathbf{S}_{1}^{*}\right) \mathbf{g}=0, \quad \mathbf{g}^{T}\left(\mathbf{S}_{1}^{T}-\mathbf{S}_{1}^{*}\right) \mathbf{g}=0, \quad \mathbf{g}^{T}\left(\mathbf{S}_{2}-\mathbf{S}_{2}^{*}\right) \mathbf{g}=0,
$$

and the secant core stiffness is not unique because

$$
\left(\mathbf{S}_{1}^{T}-\mathbf{S}_{1}^{*}\right) \mathbf{g}=\mathbf{0}, \quad\left(\mathbf{S}_{2}-\mathbf{S}_{2}^{*}\right) \mathbf{g}=\mathbf{0} .
$$

Adding "gage terms" such as those of (4.17) multiplied by arbitrary coefficients does not change $\delta \mathcal{U}$ and consequently the secant stiffness acquires two free parameters. Uniqueness holds for the tangent stiffness because the test vectors are the virtual displacement gradient variations, and $\mathbf{S}$ is not a function of $\delta \mathrm{g}$.

REMARK 4.3. Because of (4.16), an additional free parameter appears in $\mathbf{S}^{U}$ if unsymmetry is allowed. If symmetry is enforced the first two gage expressions must be combined to read $\mathbf{g}^{T}\left(\mathbf{S}_{1}+\mathbf{S}_{1}^{T}-2 \mathbf{S}_{1}^{*}\right) \mathbf{g}=\mathbf{0}$. 


\subsection{Spectral Forms}

There is a more compact alternative expression of the core stiffnesses that offers theoretical as well as implementational advantages at the cost of some generality. Define vectors $\mathbf{b}_{\boldsymbol{i}}$ and $\mathrm{c}_{i}$ as

$$
e_{i}=\mathbf{c}_{i}^{T} \mathbf{g}, \quad \mathbf{c}_{i}=\mathbf{h}_{i}+\frac{1}{2} \mathbf{H}_{i} \mathbf{g}, \quad \mathbf{b}_{i}=\frac{\partial e_{i}}{\partial \mathrm{g}}=\mathbf{h}_{i}+\mathbf{H}_{i} \mathbf{g} .
$$

Then the spectral forms (so called because of the formal similarity of equations (4.19)(4.21) with the spectral decomposition of a matrix as the sum of rank-one matrices) are

$$
\begin{gathered}
\mathbf{S}^{U}(1,1)=\left.\mathbf{S}^{U}\right|_{\alpha=\beta=1}=E_{i j} \mathbf{c}_{i} \mathbf{c}_{j}^{T}+s_{i}^{0} \mathbf{H}_{i}, \\
\mathbf{S}^{r}(0,0)=\left.\mathbf{S}^{r}\right|_{\phi=\psi=0}=E_{i j} \mathbf{b}_{i} \mathbf{c}_{j}^{T}+s_{i}^{0} \mathbf{H}_{i}, \\
\mathbf{S}^{r}\left(\frac{1}{2}, 1\right)=\left.\mathbf{S}^{r}\right|_{\phi=\frac{1}{2}, \psi=1}=E_{i j} \mathbf{c}_{i} \mathbf{c}_{j}^{T}+\frac{1}{2}\left(s_{i}+s_{i}^{0}\right) \mathbf{H}_{i}, \\
\mathbf{S}=E_{i j} \mathbf{b}_{i} \mathbf{b}_{j}^{T}+s_{i} \mathbf{H}_{i}=\mathbf{S}_{M}+\mathbf{S}_{G} .
\end{gathered}
$$

Note that $\mathbf{S}^{r}\left(\frac{1}{2}, 1\right)$ is symmetric but $\mathbf{S}^{\mathbf{r}}(0,0)$ is not. It is seen that for energy and secant stiffnesses, compactness is paid in terms of settling for specific coefficients.

REMARK 4.4 . The foregoing relations may be easily verified by noting that

$$
\begin{aligned}
E_{i j} \mathbf{c}_{i} \mathbf{c}_{j}^{T} & =\mathbf{S}_{0}+\frac{1}{2}\left(\mathbf{S}_{1}+\mathbf{S}_{1}^{T}\right)+\frac{1}{4} \mathbf{S}_{2} \\
E_{i j} \mathbf{b}_{i} \mathbf{c}_{j}^{T} & =\mathbf{S}_{0}+\frac{1}{2} \mathbf{S}_{1}+\mathbf{S}_{1}^{T}+\frac{1}{2} \mathbf{S}_{2} \\
E_{i j} \mathbf{b}_{i} \mathbf{b}_{j}^{T} & =\mathbf{S}_{0}+\mathbf{S}_{1}+\mathbf{S}_{1}^{T}+\mathbf{S}_{2} \\
E_{i j} \frac{\partial\left(\mathbf{c}_{i} \mathbf{c}_{j}^{T}\right)}{\partial \mathbf{g}} & =E_{i j}\left[\mathbf{c}_{i}\left(\frac{\partial \mathbf{c}_{j}}{\partial \mathbf{g}}\right)^{T}+\left(\frac{\partial \mathbf{c}_{i}}{\partial \mathbf{g}}\right) \mathbf{c}_{j}^{T}\right]=\mathbf{S}_{1}^{*}+\frac{1}{2} \mathbf{S}_{2}^{*}=E_{i j} e_{j} \mathbf{H}_{i}=\left(s_{i}-s_{i}^{0}\right) \mathbf{H}_{i} \\
E_{i j} \frac{\partial^{2}\left(\mathbf{c}_{i} \mathbf{c}_{j}^{T}\right)}{\partial \mathbf{g}^{2}} & =2 E_{i j} \frac{\partial \mathbf{c}_{i}}{\partial \mathbf{g}}\left(\frac{\partial \mathbf{c}_{j}}{\partial \mathbf{g}}\right)^{T}=\frac{1}{2} \mathbf{S}_{2}^{*}
\end{aligned}
$$

and seeking these patterns in the general parametrized expressions (4.13).

\subsection{Generalization to $\mathrm{H}(\mathrm{g})$}

If the $\mathbf{H}_{i}$ depend on $\mathbf{g}$, as it generally happens if strain measures other than GreenLagrange's are used, the secant and tangent stiffness core equations become more complex because of the presence of first and second $\mathbf{g}$-derivatives of $\mathbf{H}_{\boldsymbol{i}}$. The changes in the core variational equations (4.7)-(4.8) can be succintly expressed as

$$
\begin{gathered}
\delta \mathcal{U}=\delta \mathbf{g}^{T}\left(\left(\mathbf{S}^{r}+\widehat{\mathbf{S}}^{r}\right) \mathbf{g}+\Psi^{0}+\widehat{\mathbf{\Psi}}^{0}\right)=\delta \mathbf{g}^{T}(\mathbf{\Phi}+\widehat{\mathbf{\Phi}}), \\
\delta^{2} \mathcal{U}=\delta \mathbf{g}^{T}(\mathbf{S}+\widehat{\mathbf{S}}) \delta \mathbf{g}+\left(\delta^{2} \mathbf{g}\right)^{T}(\mathbf{\Phi}+\widehat{\mathbf{\Phi}}) .
\end{gathered}
$$


where $\widehat{\mathbf{S}}^{r}, \widehat{\mathbf{S}}$ and $\widehat{\mathbf{\Phi}}$ are additional core terms that arise on account of the dependence of the $\mathbf{H}_{i}$ on $\mathbf{g}$.

The parametrization and efficient characterization of such terms for several strain measures of interest in practice, notably logarithmic and midpoint strains, are presently open problems. Such topics would in fact be good candidates for term projects in advanced nonlinear finite element courses.

\section{CORE STIFFNESS EXAMPLES}

Because the core equations reflect the motion of an individual particle, their form is primarily determined by the choice of components of $\mathbf{s}$, $\mathbf{e}$ and $\mathbf{g}$ that are retained in the strain energy density. This choice is in turn a byproduct of the mathematical idealization of the actual structure or structural component.

Several cases are worked out below to illustrate the basic steps. The core expressions developed in these examples do not force commitment to specific elements, only to a mathematical model. For example the bar core equations may be subsequently used to develop 2-node straight elements or 3-node curved ones. Some specific elements based on these equations are derived in Sections 7, 9 and 10.

\subsection{Bar in 3D Space}

The particle belongs to a bar moving in 3D space. The only energy contribution is due to the longitudinal stress. We have $n_{d}=3, n_{s}=1$ and $n_{g}=3$. To simplify node subscripting, Cartesian systems and displacement components will be denoted by $\{X, Y, Z\},\{x, y, z\}$ and $\left\{u_{X}, u_{Y}, u_{Z}\right\}$ rather than $\left\{X_{1}, X_{2}, X_{3}\right\},\left\{x_{1}, x_{2}, x_{3}\right\}$ and $\left\{u_{1}, u_{2}, u_{3}\right\}$, respectively. In the reference configuration $\mathcal{C}_{0}$ the bar is referred to a local Cartesian system $\{\bar{X}, \bar{Y}, \bar{Z}\}$, with $\bar{X}$ located along the bar axis.

With reference to this local system, the motion of a particle initially at $\bar{X}$ is defined by the displacement components $\bar{u}_{X}=\bar{u}_{X}(\bar{X}), \bar{u}_{Y}=\bar{u}_{Y}(\bar{X})$ and $\bar{u}_{Z}=\bar{u}_{Z}(\bar{X})$. The three displacement gradients that intervene in the definition of nonlinear strains are

$$
\mathbf{g}=\left\{\begin{array}{l}
g_{1} \\
g_{2} \\
g_{3}
\end{array}\right\}=\left\{\begin{array}{l}
\partial \bar{u}_{X} / \partial \bar{X} \\
\partial \bar{u}_{Y} / \partial \bar{X} \\
\partial \bar{u}_{Z} / \partial \bar{X}
\end{array}\right\} .
$$

As uniaxial strain measure we adopt the Green-Lagrange (GL) axial strain, defined as

$$
\begin{aligned}
e \equiv e_{1} & =\frac{\partial \bar{u}_{X}}{\partial \bar{X}}+\frac{1}{2}\left[\left(\frac{\partial \bar{u}_{X}}{\partial \bar{X}}\right)^{2}+\left(\frac{\partial \bar{u}_{Y}}{\partial \bar{X}}\right)^{2}+\left(\frac{\partial \bar{u}_{Z}}{\partial \bar{X}}\right)^{2}\right]=g_{1}+\frac{1}{2}\left(g_{1}^{2}+g_{2}^{2}+g_{3}^{2}\right) \\
& =\left[\begin{array}{l}
1 \\
0 \\
0
\end{array}\right]^{T}\left\{\begin{array}{l}
g_{1} \\
g_{2} \\
g_{3}
\end{array}\right\}+\frac{1}{2}\left\{\begin{array}{l}
g_{1} \\
g_{2} \\
g_{3}
\end{array}\right\}^{T}\left[\begin{array}{lll}
1 & 0 & 0 \\
0 & 1 & 0 \\
0 & 0 & 1
\end{array}\right]\left\{\begin{array}{l}
g_{1} \\
g_{2} \\
g_{3}
\end{array}\right\}=\mathbf{h}^{T} \mathbf{g}+\frac{1}{2} \mathbf{g}^{T} \mathbf{H g} .
\end{aligned}
$$


Thus for this choice of strain, $\mathbf{h}_{1}^{T} \equiv \mathbf{h}^{T}=\left[\begin{array}{lll}1 & 0 & 0\end{array}\right]$ and $\mathbf{H}_{1} \equiv \mathbf{H}$ is the $\mathbf{3} \times \mathbf{3}$ identity matrix. The conjugate stress measure $s_{1} \equiv s$ is the second Piola-Kirchhoff (PK2) axial stress. The stress-strain relation is $s=s^{0}+E e$, where $s^{0}$ and $s$ are PK2 axial stresses in the reference and current configurations, respectively, and $E$ is Young's modulus.

Because $\mathbf{H}$ is independent of $\mathbf{g}$, to form the core stiffnesses in local coordinates we can directly use the spectral expressions (4.19)-(4.22). First construct the vectors

$$
\mathbf{c} \equiv \mathbf{c}_{1}=\left\{\begin{array}{c}
1+\frac{1}{2} g_{1} \\
\frac{1}{2} g_{2} \\
\frac{1}{2} g_{3}
\end{array}\right\}, \quad \mathbf{b} \equiv \mathbf{b}_{1}=\left\{\begin{array}{c}
1+g_{1} \\
g_{2} \\
g_{3}
\end{array}\right\}
$$

which inserted into the spectral forms yield

$$
\begin{gathered}
\mathbf{S}^{U}(1,1)=E \mathbf{c c}^{T}+s^{0} \mathbf{H}=E\left[\begin{array}{ccc}
\left(1+\frac{1}{2} g_{1}\right)^{2} & \frac{1}{2} g_{2}\left(1+\frac{1}{2} g_{1}\right) & \frac{1}{2} g_{3}\left(1+\frac{1}{2} g_{1}\right) \\
& \frac{1}{4} g_{2}^{2} & \frac{1}{4} g_{2} g_{3} \\
s y m m & & \frac{1}{4} g_{3}^{2}
\end{array}\right]+s^{0}\left[\begin{array}{lll}
1 & 0 & 0 \\
0 & 1 & 0 \\
0 & 0 & 1
\end{array}\right], \\
\mathbf{S}^{r}\left(\frac{1}{2}, 1\right)=E \mathbf{c c}^{T}+s^{m} \mathbf{H}=E\left[\begin{array}{ccc}
\left(1+\frac{1}{2} g_{1}\right)^{2} & \frac{1}{2} g_{2}\left(1+\frac{1}{2} g_{1}\right) & \frac{1}{2} g_{3}\left(1+\frac{1}{2} g_{1}\right) \\
& \frac{1}{4} g_{2}^{2} & \frac{1}{4} g_{2} g_{3} \\
s y m m & \frac{1}{4} g_{3}^{2}
\end{array}\right]+s^{m}\left[\begin{array}{lll}
1 & 0 & 0 \\
0 & 1 & 0 \\
0 & 0 & 1
\end{array}\right], \\
\mathbf{S}=E \mathbf{b b}^{T}+s \mathbf{H}=E\left[\begin{array}{ccc}
\left(1+g_{1}\right)^{2} & g_{2}\left(1+g_{1}\right) & g_{3}\left(1+g_{1}\right) \\
& g_{2}^{2} & g_{2} g_{3} \\
s y m m & & g_{3}^{2}
\end{array}\right]+s\left[\begin{array}{lll}
1 & 0 & 0 \\
0 & 1 & 0 \\
0 & 0 & 1
\end{array}\right] .
\end{gathered}
$$

In equation (5.5), $s^{m}=\frac{1}{2}\left(s^{0}+s\right)=s^{0}+\frac{1}{2} E e$ is the average or "half-way" stress. The clean separation into material and geometric (initial-stress) stiffnesses should be noted.

\subsection{Plate in Plane Stress}

As second example we consider a particle that pertains to a plate in plane stress (membrane), constrained to move in its plane. As usual we consider only the motion of the midplane. The Cartesian reference system and displacement components will be denoted by $\{X, Y\},\{x, y\}$ and $\left\{u_{X}, u_{Y}\right\}$ rather than $\left\{X_{1}, X_{2}\right\},\left\{x_{1}, x_{2}\right\}$ and $\left\{u_{1}, u_{2}\right\}$, respectively. The element displacement field of a generic particle originally at $(X, Y)$ is defined by the two components $u_{X}=u_{X}(X, Y)$ and $u_{Y}=u_{Y}(X, Y)$. Three in-plane PK2 stresses contribute to the strain energy and four displacement gradients appear in the corresponding GL strain. Consequently $n_{d}=2, n_{s}=3$ and $n_{g}=4$. The four displacement gradients are arranged as

$$
\mathbf{g}=\left\{\begin{array}{l}
g_{1} \\
g_{2} \\
g_{3} \\
g_{4}
\end{array}\right\}=\left\{\begin{array}{l}
\partial u_{X} / \partial X \\
\partial u_{Y} / \partial X \\
\partial u_{X} / \partial Y \\
\partial u_{Y} / \partial Y
\end{array}\right\}
$$


The strain measures chosen are the three components $e_{i}(i=1,2,3)$ of the GL strains defined in the usual manner:

$$
\begin{gathered}
e_{1}=e_{X X}=g_{1}+\frac{1}{2}\left(g_{1}^{2}+g_{2}^{2}\right)=\left\{\begin{array}{l}
1 \\
0 \\
0 \\
0
\end{array}\right\}^{T} \mathbf{g}+\frac{1}{2} \mathbf{g}^{T}\left[\begin{array}{llll}
1 & 0 & 0 & 0 \\
0 & 1 & 0 & 0 \\
0 & 0 & 0 & 0 \\
0 & 0 & 0 & 0
\end{array}\right] \mathbf{g}, \\
e_{2}=e_{Y Y}=g_{4}+\frac{1}{2}\left(g_{3}^{2}+g_{4}^{2}\right)=\left\{\begin{array}{l}
0 \\
0 \\
0 \\
1
\end{array}\right\}^{T} \mathbf{g}+\frac{1}{2} \mathbf{g}^{T}\left[\begin{array}{llll}
0 & 0 & 0 & 0 \\
0 & 0 & 0 & 0 \\
0 & 0 & 1 & 0 \\
0 & 0 & 0 & 1
\end{array}\right] \mathbf{g}, \\
e_{3}=e_{X Y}+e_{Y X}=g_{2}+g_{3}+g_{1} g_{3}+g_{2} g_{4}=\left\{\begin{array}{l}
0 \\
1 \\
1 \\
0
\end{array}\right\}^{T} \mathbf{g}+\frac{1}{2} \mathbf{g}^{T}\left[\begin{array}{llll}
0 & 0 & 1 & 0 \\
0 & 0 & 0 & 1 \\
1 & 0 & 0 & 0 \\
0 & 1 & 0 & 0
\end{array}\right] \mathbf{g},
\end{gathered}
$$

from which expressions for $\mathbf{h}_{i}$ and $\mathbf{H}_{i}(i=1,2,3)$ follow. For brevity, only the derivation of the tangent stiffness matrix will be described. Begin by forming the vectors

$$
\mathbf{b}_{1}=\left\{\begin{array}{c}
1+g_{1} \\
g_{2} \\
0 \\
0
\end{array}\right\}, \quad \mathbf{b}_{2}=\left\{\begin{array}{c}
0 \\
0 \\
g_{3} \\
1+g_{4}
\end{array}\right\}, \quad \mathbf{b}_{3}=\left\{\begin{array}{c}
g_{3} \\
1+g_{4} \\
1+g_{1} \\
g_{2}
\end{array}\right\}
$$

Then from (4.22) we get the core stiffness

$$
\mathbf{S}=E_{i j} \mathbf{b}_{i} \mathbf{b}_{i}^{T}+s_{i} \mathbf{H}_{i}=\mathbf{S}_{M}+\mathbf{S}_{G},
$$

where $s_{i}=s_{i}^{0}+E_{i j} e_{j},(i, j=1,2,3)$, are the PK2 stresses in the current configuration. In full and using the abbreviations $a_{1}=1+g_{1}, a_{4}=1+g_{4}$ we get

$$
\mathbf{S}_{M}=\left[\begin{array}{cc}
E_{11} a_{1}^{2}+2 E_{13} a_{1} g_{3}+E_{33} g_{3}^{2} & E_{11} a_{1} g_{2}+E_{13}\left(a_{1} a_{4}+g_{2} g_{3}\right)+E_{33} a_{4} g_{3} \\
\multicolumn{3}{c}{E_{11} g_{2}^{2}+2 E_{13} a_{4} g_{2}+E_{33} a_{4}^{2}} \\
s y m m \\
E_{12} a_{1} g_{3}+E_{13} a_{1}^{2}+E_{23} g_{3}^{2}+E_{33} a_{1} g_{3} & E_{12} a_{1} a_{4}+E_{13} a_{1} g_{2}+E_{23} a_{4} g_{3}+E_{33} g_{2} g_{3} \\
E_{12} g_{2} g_{3}+E_{13} a_{1} g_{2}+E_{23} a_{4} g_{3}+E_{33} a_{1} a_{4} & E_{12} a_{4} g_{2}+E_{13} g_{2}^{2}+E_{23} a_{4}^{2}+E_{33} a_{4} g_{2} \\
E_{22} g_{3}^{2}+2 E_{23} a_{1} g_{3}+E_{33} a_{1}^{2} & E_{22} a_{4} g_{3}+E_{23}\left(a_{1} a_{4}+g_{2} g_{3}\right)+E_{33} a_{1} g_{2} \\
& E_{22} a_{4}^{2}+2 E_{23} a_{4} g_{2}+E_{33} g_{2}^{2}
\end{array}\right]
$$

$$
\mathbf{S}_{G}=\left[\begin{array}{cccc}
s_{1} & 0 & s_{3} & 0 \\
& s_{1} & 0 & s_{3} \\
& & s_{2} & 0 \\
\text { symm } & & & s_{2}
\end{array}\right]
$$




\subsection{Plate Bending}

This is similar to the previous example in that the structure is a flat thin plate but now motion in $3 \mathrm{D}$ space $\{X, Y, Z\}$ is allowed. With this increased freedom the plate is capable of membrane stretching and bending. For the latter a Kirchhoff mathematical model is assumed. The three energy-contributing GL strains are now functions of six gradients. Consequently $n_{d}=3, n_{s}=3$ and $n_{g}=6$. The contributing gradients are arranged as

$$
\mathbf{g}=\left\{\begin{array}{l}
g_{1} \\
g_{2} \\
g_{3} \\
g_{4} \\
g_{5} \\
g_{6}
\end{array}\right\}=\left\{\begin{array}{l}
\partial u_{X} / \partial X \\
\partial u_{Y} / \partial X \\
\partial u_{Z} / \partial Z \\
\partial u_{X} / \partial Y \\
\partial u_{Y} / \partial Y \\
\partial u_{Z} / \partial Y
\end{array}\right\}
$$

The three GL strains are defined as

$$
\begin{aligned}
& e_{1}=e_{X X}=g_{1}+\frac{1}{2}\left(g_{1}^{2}+g_{2}^{2}+g_{3}^{2}\right)=\left\{\begin{array}{l}
1 \\
0 \\
0 \\
0 \\
0 \\
0
\end{array}\right\} \mathbf{g}+\frac{1}{2} \mathbf{g}^{T}\left[\begin{array}{llllll}
1 & 0 & 0 & 0 & 0 & 0 \\
0 & 1 & 0 & 0 & 0 & 0 \\
0 & 0 & 1 & 0 & 0 & 0 \\
0 & 0 & 0 & 0 & 0 & 0 \\
0 & 0 & 0 & 0 & 0 & 0 \\
0 & 0 & 0 & 0 & 0 & 0
\end{array}\right] \mathbf{g}, \quad(5.16) \\
& e_{2}=e_{Y Y}=g_{5}+\frac{1}{2}\left(g_{4}^{2}+g_{5}^{2}+g_{6}^{2}\right)=\left\{\begin{array}{l}
0 \\
0 \\
0 \\
0 \\
1 \\
0
\end{array}\right\} \mathbf{g}+\frac{1}{2} \mathbf{g}^{T}\left[\begin{array}{llllll}
0 & 0 & 0 & 0 & 0 & 0 \\
0 & 0 & 0 & 0 & 0 & 0 \\
0 & 0 & 0 & 0 & 0 & 0 \\
0 & 0 & 0 & 1 & 0 & 0 \\
0 & 0 & 0 & 0 & 1 & 0 \\
0 & 0 & 0 & 0 & 0 & 1
\end{array}\right] \mathbf{g}, \quad(5.17) \\
& e_{3}=e_{X Y}+e_{Y X}=g_{2}+g_{4}+g_{1} g_{4}+g_{2} g_{5}+g_{3} g_{6}=\left\{\begin{array}{l}
0 \\
1 \\
0 \\
1 \\
0 \\
0
\end{array}\right\} \mathbf{g}+\frac{1}{2} \mathbf{g}^{T}\left[\begin{array}{llllll}
0 & 0 & 0 & 1 & 0 & 0 \\
0 & 0 & 0 & 0 & 1 & 0 \\
0 & 0 & 0 & 0 & 0 & 1 \\
1 & 0 & 0 & 0 & 0 & 0 \\
0 & 1 & 0 & 0 & 0 & 0 \\
0 & 0 & 1 & 0 & 0 & 0
\end{array}\right] \mathbf{g},
\end{aligned}
$$

which define $\mathbf{h}_{i}$ and $\mathbf{H}_{i}, i=1,2,3$. When one reaches this level of bookkeeping it is more expedient and less error-prone to obtain the core matrices through symbolic manipulation. For example, the following Macsyma program forms $\mathbf{S}_{M}$ and $\mathbf{S}_{G}$ in matrices SM and SG, respectively:

$$
\begin{aligned}
& \mathrm{h} 1: \operatorname{matrix}([1],[0],[0],[0],[0],[0]) \$ \\
& \mathrm{~h} 2: \operatorname{matrix}([0],[0],[0],[0],[1],[0]) \$
\end{aligned}
$$




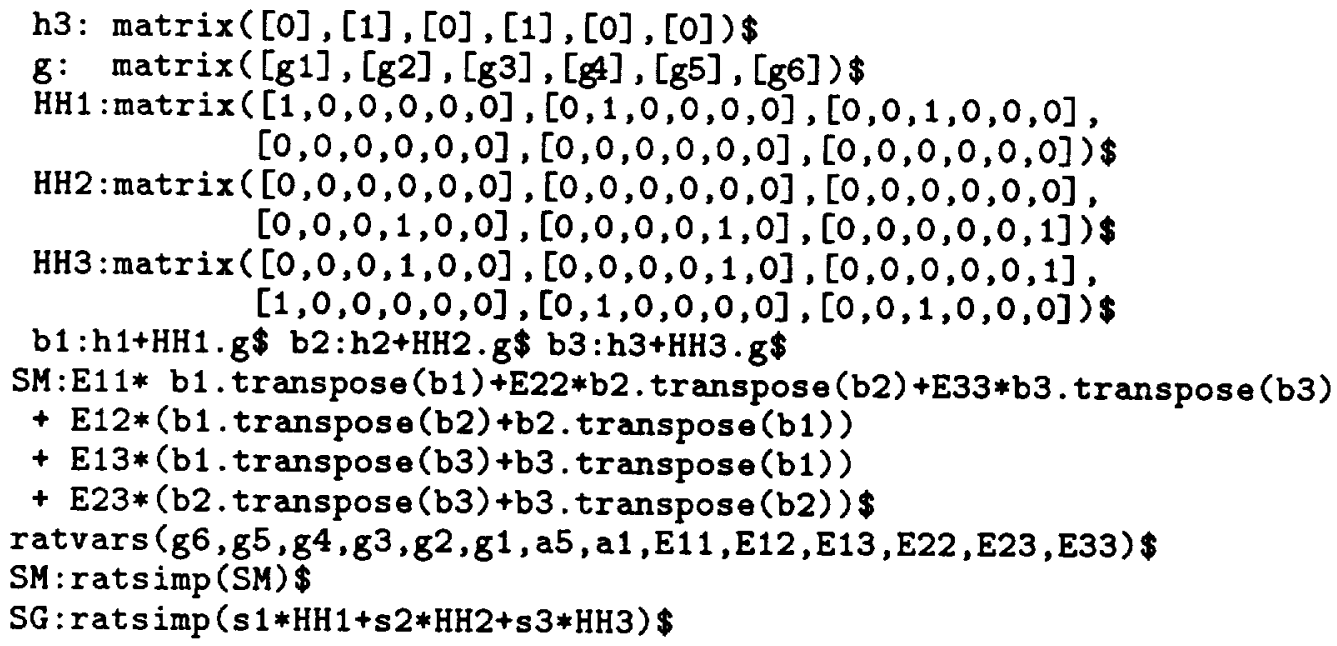

These matrices may be automatically converted to TEX by appropriate Macsyma statements (not shown above). That output was reformatted by hand for inclusion here. For the core tangent stiffness this semi-automated process yields

$$
\begin{aligned}
& S_{M}(1,1)=E_{33} g_{4}^{2}+2 E_{13}\left(1+g_{1}\right) g_{4}+E_{11}\left(1+g_{1}\right)^{2} \\
& S_{M}(1,2)=E_{13}\left(\left(1+g_{1}\right)\left(1+g_{5}\right)+g_{2} g_{4}\right)+E_{33} g_{4}\left(1+g_{5}\right)+E_{11}\left(1+g_{1}\right) g_{2} \\
& S_{M}(1,3)=E_{13}\left(\left(1+g_{1}\right) g_{6}+g_{3} g_{4}\right)+E_{33} g_{4} g_{6}+E_{11}\left(1+g_{1}\right) g_{3} \\
& S_{M}(1,4)=E_{23} g_{4}^{2}+E_{33}\left(1+g_{1}\right) g_{4}+E_{12}\left(1+g_{1}\right) g_{4}+E_{13}\left(1+g_{1}\right)^{2} \\
& S_{M}(1,5)=E_{12}\left(\left(1+g_{1}\right)\left(1+g_{5}\right)\right)+E_{23} g_{4}\left(1+g_{5}\right)+E_{33} g_{2} g_{4}+E_{13}\left(1+g_{1}\right) g_{2} \\
& S_{M}(1,6)=E_{12}\left(1+g_{1}\right) g_{6}+E_{23} g_{4} g_{6}+E_{33} g_{3} g_{4}+E_{13}\left(1+g_{1}\right) g_{3} \\
& S_{M}(2,2)=E_{33}\left(1+g_{5}\right)^{2}+2 E_{13} g_{2}\left(1+g_{5}\right)+E_{11} g_{2}^{2} \\
& S_{M}(2,3)=E_{33}\left(1+g_{5}\right) g_{6}+E_{13}\left(g_{2} g_{6}+g_{3}\left(1+g_{5}\right)\right)+E_{11} g_{2} g_{3} \\
& S_{M}(2,4)=E_{33}\left(\left(1+g_{1}\right)\left(1+g_{5}\right)\right)+E_{23} g_{4}\left(1+g_{5}\right)+E_{12} g_{2} g_{4}+E_{13}\left(1+g_{1}\right) g_{2} \\
& S_{M}(2,5)=E_{23}\left(1+g_{5}\right)^{2}+E_{33} g_{2}\left(1+g_{5}\right)+E_{12} g_{2}\left(1+g_{5}\right)+E_{13} g_{2}^{2} \\
& S_{M}(2,6)=E_{23}\left(1+g_{5}\right) g_{6}+E_{12} g_{2} g_{6}+E_{33} g_{3}\left(1+g_{5}\right)+E_{13} g_{2} g_{3} \\
& S_{M}(3,3)=E_{33} g_{6}^{2}+2 E_{13} g_{3} g_{6}+E_{11} g_{3}^{2} \\
& S_{M}(3,4)=E_{33}\left(1+g_{1}\right) g_{6}+E_{23} g_{4} g_{6}+E_{12} g_{3} g_{4}+E_{13}\left(1+g_{1}\right) g_{3} \\
& S_{M}(3,5)=E_{23}\left(1+g_{5}\right) g_{6}+E_{33} g_{2} g_{6}+E_{12} g_{3}\left(1+g_{5}\right)+E_{13} g_{2} g_{3} \\
& S_{M}(3,6)=E_{23} g_{6}^{2}+E_{33} g_{3} g_{6}+E_{12} g_{3} g_{6}+E_{13} g_{3}^{2} \\
& S_{M}(4,4)=E_{22} g_{4}^{2}+2 E_{23}\left(1+g_{1}\right) g_{4}+E_{33}\left(1+g_{1}\right)^{2} \\
& S_{M}(4,5)=E_{23}\left(\left(1+g_{1}\right)\left(1+g_{5}\right)+g_{2} g_{4}\right)+E_{22} g_{4}\left(1+g_{5}\right)+E_{33}\left(1+g_{1}\right) g_{2} \\
& S_{M}(4,6)=E_{23}\left(\left(1+g_{1}\right) g_{6}+g_{3} g_{4}\right)+E_{22} g_{4} g_{6}+E_{33}\left(1+g_{1}\right) g_{3} \\
& S_{M}(5,5)=E_{22}\left(1+g_{5}\right)^{2}+2 E_{23} g_{2}\left(1+g_{5}\right)+E_{33} g_{2}^{2} \\
& S_{M}(5,6)=E_{22}\left(1+g_{5}\right) g_{6}+E_{23}\left(g_{2} g_{6}+g_{3}\left(1+g_{5}\right)\right)+E_{33} g_{2} g_{3} \\
& S_{M}(6,6)=E_{22} g_{6}^{2}+2 E_{23} g_{3} g_{6}+E_{33} g_{3}^{2}
\end{aligned}
$$

(which can be further compacted by introducing the auxiliary symbols $a_{1}=1+g_{1}$ and 


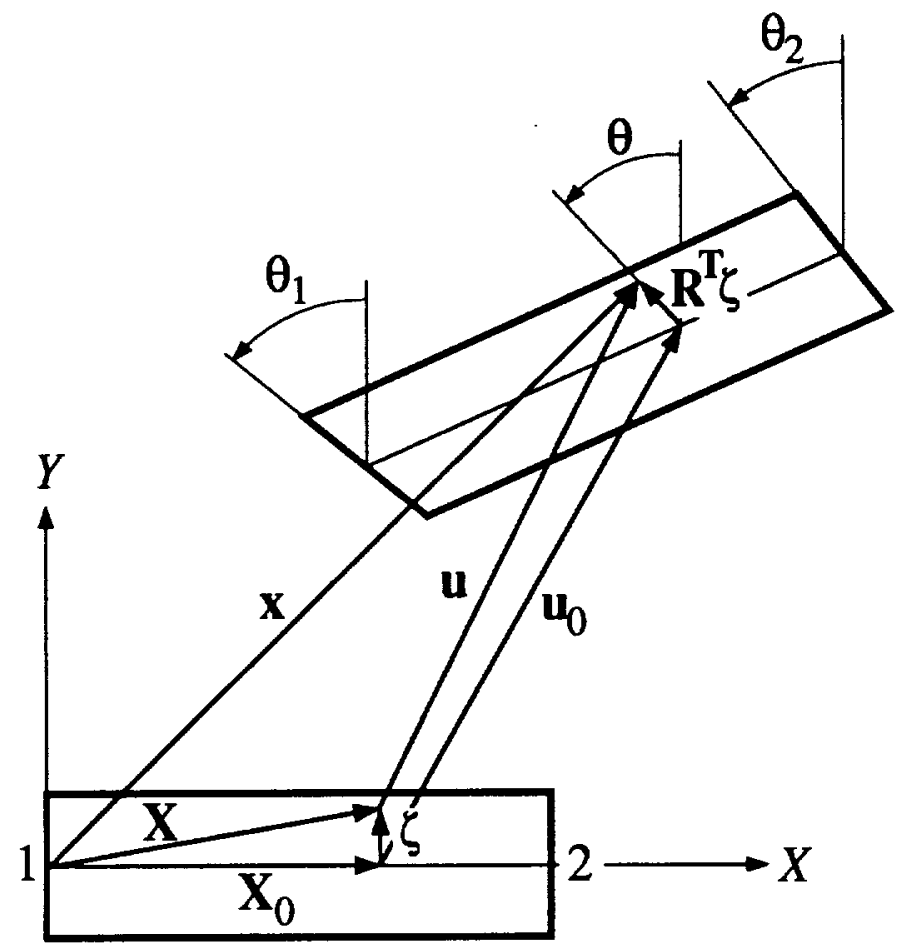

Figure 1 Kinematics of 2D Timoshenko beam element

$a_{5}=1+g_{5}$ as done in Section 5.3) and

$$
\mathbf{S}_{G}=\mathbf{S}_{G P}=\left[\begin{array}{cccccc}
s_{1} & 0 & 0 & s_{3} & 0 & 0 \\
0 & s_{1} & 0 & 0 & s_{3} & 0 \\
0 & 0 & s_{1} & 0 & 0 & s_{3} \\
s_{3} & 0 & 0 & s_{2} & 0 & 0 \\
0 & s_{3} & 0 & 0 & s_{2} & 0 \\
0 & 0 & s_{3} & 0 & 0 & s_{2}
\end{array}\right]
$$

REMARK 5.1. If the plate element to which the particle belong has (as usual) rotational freedoms, an additional geometric stiffness (the complementary geometric stiffness) appears in the transformation phase. Because of this, the core geometric stiffness $(5.20)$ has been relabeled as $\mathbf{S}_{G P}$, where subscript $P$ means "principal."

REMARK 5.2. The core stiffness matrices may also be used for part of the formulation of thinshell facet elements, with the proviso that global reference axes $\{X, Y, Z\}$ are to be replaced by a local coordinate system $\{\bar{X}, \bar{Y}, \bar{Z}\}$ with $\bar{Z}$ normal to the element midplane.

\subsection{D Timoshenko Beam}

Consider next an isotropic Timoshenko plane beam that moves in the $(X, Y)$ plane. For notational simplicity it is assumed that the longitudinal axis of the beam is aligned with $X$. The only PK2 stresses that contribute to the strain energy are the axial stress $s_{1} \equiv s_{X X}$ and the mean shear stress $s_{2} \equiv s_{X Y}$. The corresponding GL strains are the axial strain 
$e_{1} \equiv e_{X X}$ and the section-averaged shear strain $e_{2} \equiv \gamma_{X Y}=e_{X Y}+e_{Y X}$. The constitutive equations are $s_{1}=s_{1}^{0}+E e_{1}$ and $s_{2}=s_{2}^{0}+G e_{2}$, where $E$ and $G$ are the Young's modulus and shear modulus, respectively, of the material. The treatment outlined below is slightly modified from that of a course term project by Alexander, de la Fuente and Haugen. ${ }^{10}$

The finite displacements are described in a local coordinate system that is attached to the initial position of the beam, as illustrated in Figure 1. Under the usual kinematic assumptions of the Timoshenko beam model (plane sections remain plane but not necessarily normal to the deformed centroidal axis) the coordinates of a particle in the underformed and deformed configurations may be written

$$
\begin{gathered}
\mathbf{X}=\mathbf{X}_{0}+\boldsymbol{\zeta}, \quad \mathbf{X}_{0}=\left\{\begin{array}{c}
X \\
0
\end{array}\right\}, \quad \boldsymbol{\zeta}=\left\{\begin{array}{c}
0 \\
Y
\end{array}\right\}, \\
\mathbf{x}=\mathbf{x}_{0}+\mathbf{R}^{T} \boldsymbol{\zeta}, \quad \mathbf{x}_{0}=\left\{\begin{array}{c}
X+u_{0 X} \\
u_{0} Y
\end{array}\right\}, \quad \mathbf{R}^{T}=\left[\begin{array}{cc}
\cos \theta & -\sin \theta \\
\sin \theta & \cos \theta
\end{array}\right],
\end{gathered}
$$

where $u_{0 X}$ and $u_{0 Y}$ are the components of the centroidal displacement vector $\mathbf{u}_{0}$. Subtracting (5.21) from (5.22) gives the element displacement field

$$
\mathbf{u}=\mathbf{x}-\mathbf{X}=\left\{\begin{array}{l}
u_{X} \\
u_{Y}
\end{array}\right\}=\left\{\begin{array}{c}
u_{0 X}-Y \sin \theta \\
u_{0 Y}+Y(\cos \theta-1)
\end{array}\right\} .
$$

Four displacement gradients contribute to the GL strains. Thus for this case we have $n_{d}=2, n_{s}=2$ and $n_{g}=4$. The four contributing displacement gradients are arranged in the usual pattern:

$$
\mathbf{g}=\left\{\begin{array}{l}
g_{1} \\
g_{2} \\
g_{3} \\
g_{4}
\end{array}\right\}=\left\{\begin{array}{l}
\partial u_{X} / \partial X \\
\partial u_{Y} / \partial X \\
\partial u_{X} / \partial Y \\
\partial u_{Y} / \partial Y
\end{array}\right\} .
$$

For future use in Section 9 we note that the gradients can be written in terms of generalized section freedoms as

$$
\mathbf{g}=\left\{\begin{array}{l}
g_{1} \\
g_{2} \\
g_{3} \\
g_{4}
\end{array}\right\}=\left\{\begin{array}{c}
\epsilon-Y \kappa \cos \theta \\
\gamma-Y \kappa \sin \theta \\
-\sin \theta \\
\cos \theta-1
\end{array}\right\},
$$

in which $\epsilon=\partial u_{0 X} / \partial X$ is a generalized axial strain, $\gamma=\partial u_{0 Y} / \partial X$ a generalized shear strain, and $\kappa=\partial \theta / \partial X$ is the beam curvature.

The matrix form of the GL strains is

$$
e_{1}=g_{1}+\frac{1}{2}\left(g_{1}^{2}+g_{2}\right)^{2}=\left\{\begin{array}{l}
1 \\
0 \\
0 \\
0
\end{array}\right\}^{T} \mathbf{g}+\frac{1}{2} \mathbf{g}^{T}\left[\begin{array}{llll}
1 & 0 & 0 & 0 \\
0 & 1 & 0 & 0 \\
0 & 0 & 0 & 0 \\
0 & 0 & 0 & 0
\end{array}\right] \mathbf{g},
$$




$$
e_{2}=g_{2}+g_{3}+g_{1} g_{3}+g_{2} g_{4}=\left\{\begin{array}{l}
0 \\
1 \\
1 \\
0
\end{array}\right\}^{T} \mathbf{g}+\frac{1}{2} \mathbf{g}^{T}\left[\begin{array}{llll}
0 & 0 & 1 & 0 \\
0 & 0 & 0 & 1 \\
1 & 0 & 0 & 0 \\
0 & 1 & 0 & 0
\end{array}\right] \mathbf{g},
$$

which define $h_{1}, h_{2}, \mathbf{H}_{1}$ and $\mathbf{H}_{2}$. On introducing the auxiliary vectors

$$
\mathbf{b}_{1}=\left\{\begin{array}{c}
1+g_{1} \\
g_{2} \\
0 \\
0
\end{array}\right\}, \quad \mathbf{b}_{2}=\left\{\begin{array}{c}
g_{3} \\
1+g_{4} \\
1+g_{1} \\
g_{2}
\end{array}\right\}, \quad \mathbf{c}_{1}=\left\{\begin{array}{c}
1+\frac{1}{2} g_{1} \\
\frac{1}{2} g_{2} \\
0 \\
0
\end{array}\right\}, \quad \mathbf{c}_{2}=\left\{\begin{array}{c}
\frac{1}{2} g_{3} \\
1+\frac{1}{2} g_{4} \\
1+\frac{1}{2} g_{1} \\
\frac{1}{2} g_{2}
\end{array}\right\} \text {, }
$$

the spectral core stiffness matrices and internal force vector can be written

$$
\begin{aligned}
& \mathbf{S}^{U}=E \mathbf{c}_{1} \mathbf{c}_{1}^{T}+G \mathbf{c}_{2} \mathbf{c}_{2}^{T}+s_{1}^{0} \mathbf{H}_{1}+s_{2}^{0} \mathbf{H}_{2}, \\
& \mathbf{S}^{r}=E \mathbf{c}_{1} \mathbf{c}_{1}^{T}+G \mathbf{c}_{2} \mathbf{c}_{2}^{T}+\frac{1}{2}\left(s_{1}^{0}+s_{1}\right) \mathbf{H}_{1}+\frac{1}{2}\left(s_{2}^{0}+s_{2}\right) \mathbf{H}_{2}, \\
& \mathbf{S}=\mathbf{S}_{M}+\mathbf{S}_{G P}, \quad \mathbf{S}_{M}=E \mathbf{b}_{1} \mathbf{b}_{1}^{T}+G \mathbf{b}_{2} \mathbf{b}_{2}^{T}, \quad \mathbf{S}_{G P}=s_{1} \mathbf{H}_{1}+s_{2} \mathbf{H}_{2}, \\
& \boldsymbol{\Phi}=s_{1} \mathbf{b}_{1}+s_{2} \mathbf{b}_{2} \text {. }
\end{aligned}
$$

Because beam elements have rotational freedoms, a complementary geometric stiffness matrix appears when carrying out the transformation phase. This term is considered in the subsequent GCCF treatment of this element in Section 9.

\subsection{D Timoshenko Beam: Kinematics}

The last example of derivation of core equations involve a TL 3D Timoshenko beam capable of arbitrarily large rotations. The following material is largely extracted from a recent paper by Crivelli and Felippa ${ }^{7}$ as well as Crivelli's thesis ${ }^{5}$ and is continued with the DGCCF transformation phase in Section 10. The notation used in those references has been slightly edited to fit that of the present article.

As in the $2 \mathrm{D}$ case, the beam is isotropically elastic with Young's modulus $E$ and shear modulus $G$. The reference configuration of the beam is straight and prismatic although not necessarily stress free. A local reference frame $\mathbf{n}_{i}$ is attached to it, with $\mathbf{n}_{1}$ directed along the longitudinal axis (the locus of cross section centroids). Axes $\mathbf{n}_{2}$ and $\mathbf{n}_{3}$ are in the plane of the left-end cross section; these will be eventually aligned with the principal inertia axes to simplify some algebraic expressions. Along these axes we attach the coordinate system $\{X, Y, Z\}$. This description is schematically shown in Figure 2 . We further define a set of moving frames, denoted by $\left\{\mathbf{a}_{1}, \mathbf{a}_{2}, \mathbf{a}_{3}\right\}$, parametrized by the longitudinal coordinate $X$. Initially these frames coincide with $\left\{\mathbf{n}_{1}, \mathbf{n}_{2}, \mathbf{n}_{3}\right\}$, and displace rigidly attached to the cross-sections of the moving current configuration.

A beam particle originally at $(X, Y, Z)$ displaces to

$$
\mathbf{x}(\mathbf{X})=\mathbf{x}_{0}(X)+\mathbf{R}^{T}(X) \boldsymbol{\zeta}(Y, Z), \quad \boldsymbol{\zeta}^{T}=\left[\begin{array}{lll}
0 & Y & Z
\end{array}\right],
$$




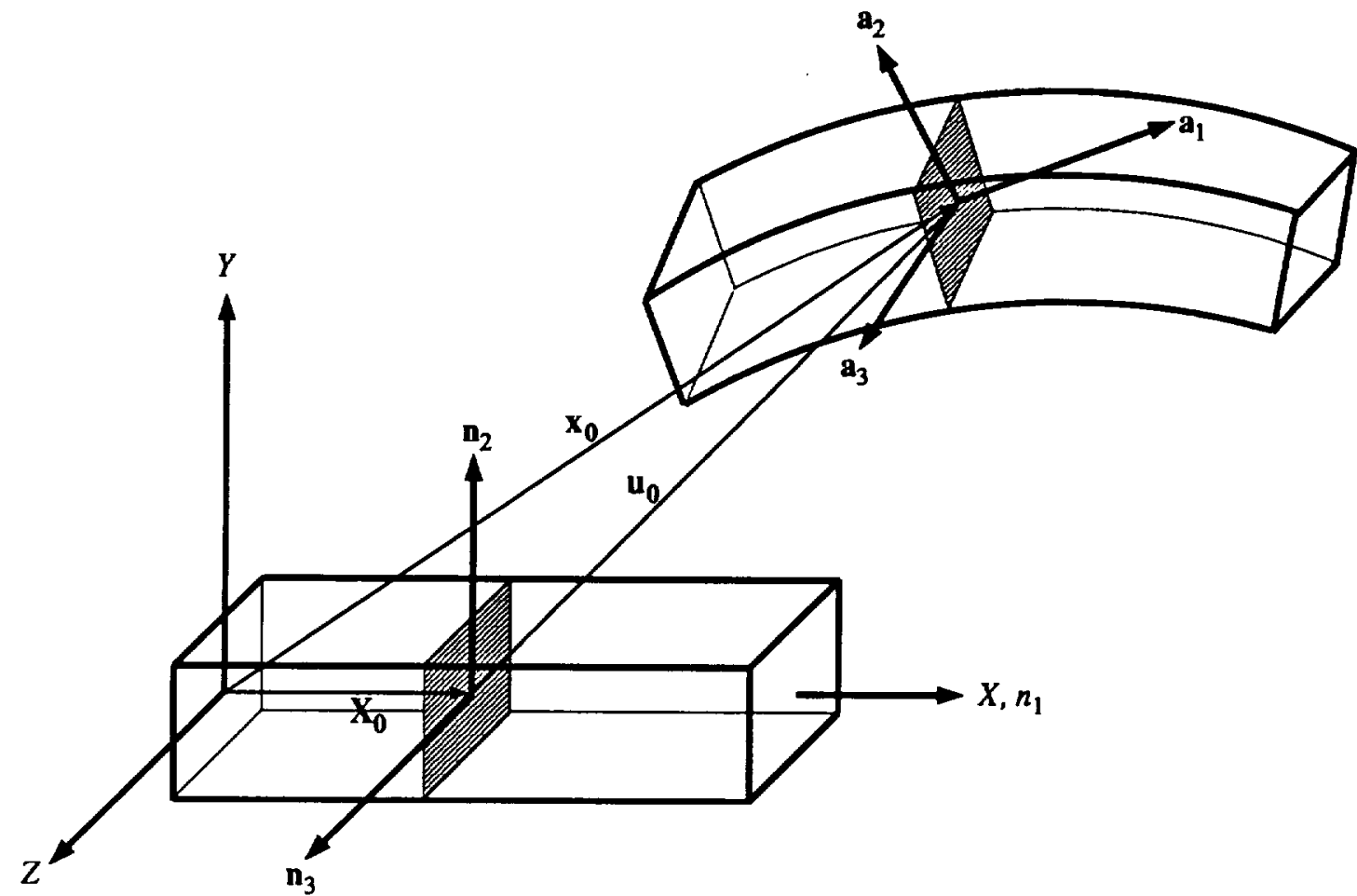

Figure 2 Kinematics of 3D Timoshenko beam element

where $\mathbf{x}_{0}$ describes the position of the centroid of the given cross-section, $\mathbf{R}$ is a 3 -by-3 orthogonal matrix function that orients the displaced cross section, and $\boldsymbol{\zeta}$ is a cross-section position vector. The displacement field is

$$
\mathbf{u}=\mathbf{x}-\mathbf{X}=\mathbf{u}_{0}+\left(\mathbf{R}^{T}-\mathbf{I}\right) \boldsymbol{\zeta}
$$

where $\mathbf{u}_{0}(X)=\mathbf{x}_{0}(X)-\mathbf{X}_{0}(X)$ is the centroidal displacement (see Figure 2).

In the sequel $3 \times 3$ skew-symmetric matrices are consistently denoted by placing a tilde over their axial 3-vector symbol; for example

$$
\tilde{\mathbf{a}}=\operatorname{spin}(\mathbf{a})=\left[\begin{array}{ccc}
0 & a_{3} & -a_{2} \\
-a_{3} & 0 & a_{1} \\
a_{2} & -a_{1} & 0
\end{array}\right], \quad \mathbf{a}=\left\{\begin{array}{l}
a_{1} \\
a_{2} \\
a_{3}
\end{array}\right\}=\operatorname{axial}(\tilde{\mathbf{a}}) .
$$

The skew-symmetric curvature matrix $\tilde{\boldsymbol{\kappa}}$ is defined by $\tilde{\boldsymbol{\kappa}}=\mathbf{R}\left(d \mathbf{R}^{T} / d X\right)$, which is the rate of change of the orthogonal rotation matrix $\mathbf{R}$ with respect to the longitudinal coordinate. The curvature vector is $\kappa=\operatorname{axial}(\tilde{\boldsymbol{\kappa}})$. We shall also require later the variation of angular orientation $\delta \Theta$, defined as the axial vector of the skew matrix $\mathbf{R} \delta \mathbf{R}^{T}$ :

$$
\widetilde{\delta \boldsymbol{\Theta}}=\mathbf{R} \delta \mathbf{R}^{T}=-\delta \mathbf{R} \mathbf{R}^{T}, \quad \delta \boldsymbol{\Theta}=\operatorname{axial}(\widetilde{\delta \Theta}),
$$


All displacement gradients $g_{i j}$ appear in the GL strain measures. To maintain compactness the nine gradients are partitioned into three 3-vectors:

$$
\mathbf{g}_{1}=\left\{\begin{array}{l}
\partial u_{X} / \partial X \\
\partial u_{Y} / \partial X \\
\partial u_{Z} / \partial X
\end{array}\right\}, \quad \mathbf{g}_{2}=\left\{\begin{array}{l}
\partial u_{X} / \partial Y \\
\partial u_{Y} / \partial Y \\
\partial u_{Z} / \partial Y
\end{array}\right\}, \quad \mathbf{g}_{3}=\left\{\begin{array}{l}
\partial u_{X} / \partial Z \\
\partial u_{Y} / \partial Z \\
\partial u_{Z} / \partial Z
\end{array}\right\}
$$

The 9-component gradient vector is $\mathbf{g}^{T}=\left[\begin{array}{lll}\mathbf{g}_{1}^{T} & \mathbf{g}_{2}^{T} & \mathbf{g}_{3}^{T}\end{array}\right]$, but this symbol is not used directly here. Also introduce the 3 -vectors

$$
\mathbf{h}_{1}=\left\{\begin{array}{l}
1 \\
0 \\
0
\end{array}\right\}, \quad \mathbf{h}_{2}=\left\{\begin{array}{l}
0 \\
1 \\
0
\end{array}\right\}, \quad \mathbf{h}_{3}=\left\{\begin{array}{l}
0 \\
0 \\
1
\end{array}\right\}
$$

With the help of these quantities, explicit expressions for the displacement gradient vectors $\mathrm{g}$ can be given as

$$
\begin{aligned}
& \mathbf{g}_{1}=\frac{d \mathbf{u}_{0}}{d X}+\mathbf{R}^{T} \tilde{\boldsymbol{\kappa}} \boldsymbol{\zeta}=\frac{d \mathbf{u}_{0}}{d X}+\mathbf{R}^{T} \tilde{\zeta}^{T} \kappa, \\
& \mathbf{g}_{2}=\left(\mathbf{R}^{T}-\mathbf{I}\right) \mathbf{h}_{2}, \quad \mathbf{g}_{3}=\left(\mathbf{R}^{T}-\mathbf{I}\right) \mathbf{h}_{3} .
\end{aligned}
$$

The only nonzero components of the GL strain tensor can be written

$$
\begin{aligned}
& e_{1} \equiv e_{11}=\quad \mathbf{h}_{1}^{T} \mathbf{g}_{1}+\frac{1}{2} \mathbf{g}_{1}^{T} \mathbf{H g}_{1}, \\
& e_{2} \equiv \gamma_{12}=2 e_{12}=\mathbf{h}_{2}^{T} \mathbf{g}_{1}+\mathbf{h}_{1}^{T} \mathbf{g}_{2}+\frac{1}{2}\left(\mathbf{g}_{1}^{T} \mathbf{H g}_{2}+\mathbf{g}_{2}^{T} \mathbf{H g}_{1}\right), \\
& e_{3} \equiv \gamma_{13}=2 e_{13}=\mathbf{h}_{3}^{T} \mathbf{g}_{1}+\mathbf{h}_{1}^{T} \mathbf{g}_{3}+\frac{1}{2}\left(\mathbf{g}_{1}^{T} \mathbf{H g}_{3}+\mathbf{g}_{3}^{T} \mathbf{H g}_{1}\right),
\end{aligned}
$$

where $\mathbf{H}$ is here the $3 \times 3$ identity matrix. Note that from the orthogonality of the rotation matrix $\mathbf{R}$ we find

$$
\begin{aligned}
e_{22} & =\mathbf{h}_{2}^{T} \mathbf{g}_{2}+\frac{1}{2} \mathbf{g}_{2}^{T} \mathbf{g}_{2} \\
& =R_{22}-1+\frac{1}{2}\left(R_{21}^{2}+\left(R_{22}-1\right)^{2}+R_{23}^{2}\right)=R_{22}-1+\frac{1}{2}\left(2-2 R_{22}\right)=0, \\
2 e_{23} & =\mathbf{h}_{2}^{T} \mathbf{g}_{3}+\mathbf{h}_{3}^{T} \mathbf{g}_{2}+\mathbf{g}_{2}^{T} \mathbf{g}_{3} \\
& =R_{32}+R_{23}+R_{21} R_{31}+R_{22} R_{32}-R_{32}+R_{23} R_{33}-R_{23}=0,
\end{aligned}
$$

and similarly $e_{33}=0$. This confirms that the only nonzero strains are (5.40).

The strains (5.40) may be rewritten in a more physically suggestive form:

$$
\begin{aligned}
& e_{1}=e_{11}=e_{b}+e_{f}, \quad \gamma=\gamma_{12}+\gamma_{13}, \\
& e_{b}=\left(\frac{d \mathbf{u}_{0}}{d X}\right)^{T}\left(\mathbf{h}_{1}+\frac{1}{2} \frac{d \mathbf{u}_{0}}{d X}\right), \quad e_{2}=\gamma_{12}=\gamma_{2}+\bar{\gamma}_{2}=\mathbf{h}_{2}^{T} \phi+\mathbf{h}_{2}^{T} \tilde{\boldsymbol{\zeta}}^{T} \kappa \\
& e_{f}=\boldsymbol{\zeta}^{T} \mathcal{K}_{e}+\frac{1}{2} \kappa^{T} \tilde{\zeta} \tilde{\zeta}^{T} \kappa \simeq \boldsymbol{\zeta}^{T} \mathcal{K}_{e}, \quad e_{3}=\gamma_{13}=\gamma_{3}+\bar{\gamma}_{3}=\mathbf{h}_{3}^{T} \phi+\mathbf{h}_{3}^{T} \tilde{\zeta}^{T} \kappa .
\end{aligned}
$$

Here $e_{b}, e_{f}$ are stretching and flexural normal strains, $\gamma_{2}$ and $\gamma_{3}$ represent bending-induced shear strains, and $\bar{\gamma}_{2}, \bar{\gamma}_{3}$ are torsion-induced shear strains. The last term in $e_{f}$ represents a squared-curvature contribution to flexure, which can usually be neglected (cf. Remark $9.2)$. The strain energy stored in the current configuration is

$$
U=\int_{L_{0}} \int_{A_{0}} \mathcal{U} d A d X, \quad \text { with } \mathcal{U}=\frac{1}{2} E e_{1}^{2}+\frac{1}{2} G\left(e_{2}^{2}+e_{3}^{2}\right)+s_{1}^{0} e_{1}+s_{2}^{0} e_{12}+s_{3}^{0} e_{3} \text {. }
$$




\subsection{D Timoshenko Beam: Core equations}

The PK2 stresses associated with the GL strains (5.40) are $s_{1} \equiv s_{11}=s_{X X}, s_{2} \equiv s_{12}=$ $s_{X Y}$ and $s_{3} \equiv s_{13}=s_{X Z}$. The constitutive equations are $s_{1}=s_{1}^{0}+E e_{1}, s_{2}=s_{2}^{0}+G e_{2}$ and $s_{3}=s_{3}^{0}+G e_{3}$. The spectral core stiffnesses can be compactly expressed in terms of the vectors $\mathbf{c}_{i}=\mathbf{h}_{i}+\frac{1}{2} \mathbf{H g}_{i}$ and $\mathbf{b}_{i}=\mathbf{h}_{i}+\mathbf{H g}_{i}$ for $i=1,2,3$, where no subscript is needed in $\mathbf{H} \equiv \mathbf{I}$. Applying the spectral formulas of Section 4.4 we obtain for the $9 \times 9$ core energy stiffness

$$
\mathbf{S}^{U}=\left[\begin{array}{ccc}
E \mathbf{S}_{1}^{U}+G\left(\mathbf{S}_{2}^{U}+\mathbf{S}_{3}^{U}\right) & G \mathbf{S}_{4}^{U} & G \mathbf{S}_{5}^{U} \\
G \mathbf{S}_{4}^{U^{T}} & G \mathbf{S}_{1}^{U} & 0 \\
G \mathbf{S}_{5}^{U} & 0 & G \mathbf{S}_{1}^{U}
\end{array}\right]+\left[\begin{array}{ccc}
s_{1}^{0} \mathbf{H} & s_{2}^{0} \mathbf{H} & s_{3}^{0} \mathbf{H} \\
s_{2}^{0} \mathbf{H} & 0 & 0 \\
s_{3}^{0} \mathbf{H} & 0 & 0
\end{array}\right]
$$

where $S_{1}^{U}=\mathbf{c}_{1} \mathbf{c}_{1}^{T}, \mathbf{S}_{2}^{U}=\mathbf{c}_{2} \mathbf{c}_{2}^{T}, \mathbf{S}_{3}^{U}=\mathbf{c}_{3} \mathbf{c}_{3}^{T}, \mathbf{S}_{4}^{U}=\mathbf{c}_{2} \mathbf{c}_{1}^{T}$ and $\mathbf{S}_{5}^{U}=\mathbf{c}_{3} \mathbf{c}_{1}^{T}$. At the residual level we obtain for $\mathbf{S}^{r}$ a form similar to (5.44) except that the prestresses $s_{i}^{0}, i=1,2,3$ have to be replaced by the midpoint stresses $\frac{1}{2}\left(s_{i}^{0}+s_{i}\right)$. The internal force vector conjugate to $\delta \mathbf{g}$ is $\boldsymbol{\Phi}=\mathbf{S}^{r} \mathbf{g}+\boldsymbol{\Phi}^{0}=\boldsymbol{\Phi}_{\boldsymbol{\sigma}}+\boldsymbol{\Phi}_{r}$, in which

$$
\boldsymbol{\Phi}_{\sigma}=\left\{\begin{array}{c}
s_{1} \mathbf{b}_{1} \\
0 \\
0
\end{array}\right\} \quad \boldsymbol{\Phi}_{\tau}=\left\{\begin{array}{c}
s_{2} \mathbf{b}_{2}+s_{3} \mathbf{b}_{3} \\
s_{2} \mathbf{b}_{1} \\
s_{3} \mathbf{b}_{1}
\end{array}\right\}
$$

represent the contribution of the normal and shear stresses, respectively.

The principal core tangent stiffness matrix $\mathbf{S}=\mathbf{S}_{M}+\mathbf{S}_{G P}$ is obtained from (4.22). The material stiffness is

$$
\mathbf{S}_{M}=\left[\begin{array}{ccc}
E \mathbf{S}_{1}+G\left(\mathbf{S}_{2}+\mathbf{S}_{3}\right) & G \mathbf{S}_{4} & G \mathbf{S}_{5} \\
G \mathbf{S}_{4}^{T} & G \mathbf{S}_{1} & 0 \\
G \mathbf{S}_{5}^{T} & 0 & G \mathbf{S}_{1}
\end{array}\right]
$$

where $\mathbf{S}_{1}=\mathbf{b}_{1} \mathbf{b}_{1}^{T}, \mathbf{S}_{2}=\mathbf{b}_{2} \mathbf{b}_{2}^{T}, \mathbf{S}_{3}=\mathbf{b}_{3} \mathbf{b}_{3}^{T}, \mathbf{S}_{4}=\mathbf{b}_{2} \mathbf{b}_{1}^{T}$ and $\mathbf{S}_{5}=\mathbf{b}_{3} \mathbf{b}_{1}^{T}$. The principal geometric stiffness is

$$
\mathbf{S}_{G P}=\left[\begin{array}{ccc}
s_{1} \mathbf{H} & s_{2} \mathbf{H} & s_{3} \mathbf{H} \\
s_{2} \mathbf{H} & \mathbf{0} & \mathbf{0} \\
s_{3} \mathbf{H} & \mathbf{0} & \mathbf{0}
\end{array}\right]=\left[\begin{array}{ccc}
\left(s_{1}^{0}+E e_{1}\right) \mathbf{H} & \left(s_{2}^{0}+G e_{2}\right) \mathbf{H} & \left(s_{2}^{0}+G e_{3}\right) \mathbf{H} \\
\left(s_{2}^{0}+G e_{2}\right) \mathbf{H} & \mathbf{0} & \mathbf{0} \\
\left(s_{2}^{0}+G e_{3}\right) \mathbf{H} & \mathbf{0} & \mathbf{0}
\end{array}\right]
$$

The contribution of $\left(\delta^{2} \mathbf{g}\right)^{T} \boldsymbol{\Phi}$ to the complementary geometric stiffness depends on the target variables in the ensuing transformation phase. Because this transformation requires the DGCCF, it is taken up in Section 10 after the GCCF is discussed in Section 8. 


\section{DCCF TRANSFORMATION TO PHYSICAL FREEDOMS}

The core stiffness matrices and internal-force vector given in (4.19)-(4.22) and (4.13), respectively, pertain to material particles of the structure. The behavior of each particle is expressed in terms of its displacement gradients collected in vector $\mathbf{g}$. To create a discrete model the structure is subdivided into finite elements. Finite elements equations in terms of the physical DOFs collected in vector $\mathbf{v}$ are constructed through a combination of core-to-physical transformations and integration over element domains.

In this section we stay within the scope of the Direct CCF by assuming that the transformations between $\mathbf{g}$ and $\mathbf{v}$ are linear. Because all subsequent developments pertain to an individual element, no element identifiers are used to reduce indexing clutter.

Over an individual element the displacement field $\mathbf{u}^{T}=\left(u_{1}, u_{2}, u_{3}\right)$ is interpolated as

$$
\mathbf{u}=\mathbf{N} \mathbf{v}
$$

where $\mathbf{v}$ now collects the element node-displacement degrees of freedom (DOFs) and $\mathbf{N}=$ $\mathbf{N}\left(X_{1}, X_{2}, X_{3}\right)$ is a matrix of shape functions independent of $\mathbf{v}$. Differentiating (6.1) with respect to the $X_{i}$ and taking the first two $\mathrm{v}$ variations yields

$$
\mathbf{g}=\mathbf{G} \mathbf{v}, \quad \delta \mathbf{g}=\mathbf{G} \delta \mathbf{v}, \quad \delta^{2} \mathbf{g}=\mathbf{0},
$$

(for the last one see Remark 4.1). Invariance of the strain energy variations $\delta U$ and $\delta^{2} U$ obtained by integrating (4.7)-(4.8) over the element reference volume yields

$$
\begin{aligned}
& \mathbf{K}^{U}=\int_{V_{0}} \mathbf{G}^{T} \mathbf{S}^{U} \mathbf{G} d V_{0}, \quad \mathbf{K}^{r}=\int_{V_{0}} \mathbf{G}^{T} \mathbf{S}^{r} \mathbf{G} d V_{0}, \quad \mathbf{K}=\int_{V_{0}} \mathbf{G}^{T} \mathbf{S G} d V_{\mathbf{0}}, \\
& \mathbf{f}=\int_{V_{0}} \mathbf{G}^{T} \mathbf{\Phi} d V_{0}, \quad \mathbf{p}=\int_{V_{0}} \mathbf{G}^{T} \Psi d V_{0}, \quad \mathbf{p}^{0}=\int_{V_{0}} \mathbf{G}^{T} \Psi^{0} d V_{0},
\end{aligned}
$$

Although the dependency of $\mathbf{S}^{\text {level }}$ and $\Psi$ on $\mathbf{g}$ is not made implicit in these equations, it must be remembered that the transformation $\mathbf{g}=\mathbf{G v}$ also appears there. Because of the ensuing algebraic complexity, numerical integration is generally required unless the gradients are constant over the element.

Often $\mathbf{G}$ is expressed as a chain of transformations, some of which are position dependent and remain inside the element integral whereas others are not and may be taken outside. For example, in the bar element treated below, $\mathbf{G}=\mathbf{T} \mathbf{G}$, where $\mathbf{G}$ transforms $\mathbf{g}$ to local node displacements while $\mathbf{T}$ transforms local to global node displacements. 


\section{DCCF TRANSFORMATION EXAMPLES}

\subsection{The Bar Element}

The core equations for a geometrically nonlinear TL bar were derived in Section 5.1. These equations are now applied to the formulation of a two-node, linear-displacement, prismatic TL bar element. The element has constant reference area $A_{0}$ and initial length $L_{0}$. The two end nodes are located at $\left(X_{1}, Y_{1}, Z_{1}\right)$ and $\left(X_{2}, Y_{2}, Z_{2}\right)$, respectively. The node displacements are $\left(v_{X_{1}}, v_{Y_{1}}, v_{Z_{1}}\right)$ and $\left(v_{X_{2}}, v_{Y_{2}}, v_{Z 2}\right)$. The element displacement field in local coordinates $\{\bar{X}, \bar{Y}, \bar{Z}\}$ may be interpolated as

$$
\overline{\mathbf{u}}=\left\{\begin{array}{l}
\bar{u}_{X} \\
\bar{u}_{Y} \\
\bar{u}_{Z}
\end{array}\right\}=\left[\begin{array}{cccccc}
N_{1} & 0 & 0 & N_{2} & 0 & 0 \\
0 & N_{1} & 0 & 0 & N_{2} & 0 \\
0 & 0 & N_{1} & 0 & 0 & N_{2}
\end{array}\right]\left\{\begin{array}{l}
\bar{v}_{X 1} \\
\bar{v}_{Y 1} \\
\bar{v}_{Z 1} \\
\bar{v}_{X 2} \\
\bar{v}_{Y 2} \\
\bar{v}_{Z 2}
\end{array}\right\}=\mathbf{N} \overline{\mathbf{v}}
$$

where $N_{1}=1-\bar{X} / L_{0}$ and $N_{2}=\bar{X} / L$ are linear shape functions. Differentiating with respect to the reference coordinate we get

$$
\mathbf{G}=\frac{1}{L_{0}}\left[\begin{array}{cccccc}
-1 & 0 & 0 & 1 & 0 & 0 \\
0 & -1 & 0 & 0 & 1 & 0 \\
0 & 0 & -1 & 0 & 0 & 1
\end{array}\right] \overline{\mathbf{v}}=\frac{1}{L_{0}} \overline{\mathbf{G}} \overline{\mathbf{v}},
$$

This transformation may be applied to the core matrices and vectors derived in Section 5.1. For example, application to the core tangent stiffness (5.6) yields

$$
\overline{\mathbf{K}}=\frac{1}{L_{0}^{2}} \int_{V_{0}} \overline{\mathbf{G}}^{T} \mathbf{S} \overline{\mathbf{G}} d V_{0}=\frac{A_{0}}{L_{0}} \overline{\mathbf{G}}^{T}\left(E \mathbf{b b}^{T}+s \mathbf{H}\right) \overline{\mathbf{G}},
$$

Finally, transformation to node displacements $\left(v_{X i}, v_{Y i}, v_{Z_{i}}\right), i=1,2$ is handled in the usual manner by writing the local-to-global transformation equation

$$
\overline{\mathbf{u}}=\left\{\begin{array}{l}
\bar{u}_{X} \\
\bar{u}_{Y} \\
\bar{u}_{Z}
\end{array}\right\}=\left[\begin{array}{lll}
T_{X X} & T_{X Y} & T_{X Z} \\
T_{Y X} & T_{Y Y} & T_{Y Z} \\
T_{Z X} & T_{Z Y} & T_{Z Z}
\end{array}\right]\left\{\begin{array}{l}
u_{X} \\
u_{Y} \\
u_{Z}
\end{array}\right\}=\mathbf{T} \mathbf{u},
$$

which is valid for both end nodes giving $\overline{\mathbf{v}}_{i}=\mathbf{T} \mathbf{v}, i=1,2$. Consequently the element tangent stiffness matrix in local coordinates is given by

$$
\mathbf{K}=\frac{A_{0}}{L_{0}}\left[\begin{array}{cc}
\mathbf{T}^{T} & \mathbf{0} \\
\mathbf{0} & \mathbf{T}^{T}
\end{array}\right] \overline{\mathbf{G}}^{T}\left(E \mathbf{b b}^{T}+s \mathbf{H}\right) \overline{\mathbf{G}}\left[\begin{array}{cc}
\mathbf{T} & \mathbf{0} \\
\mathbf{0} & \mathbf{T}
\end{array}\right] .
$$

For this simple element all entries may be obtained in closed form and no numerical integration is necessary. An efficient implementation of the tangent stiffness matrix (7.5) in the form of a Fortran subroutine is given in Appendix 2. This implementation forms $\mathbf{K}$ with approximately 160 floating point operations. 


\subsection{Iso-P Plane Stress Element}

For the case of plane stress considered in Section 5.2, we shall asume that the associated finite elements are isoparametric displacement models with $n$ nodes, and that (as usual for such models) the nodal freedoms are of translational type. The transformation to physical DOFs can then be handled within the purview of the DCCF.

As in Section 5.1, the reference system, current system and in-plane displacement components are denoted by $\{X, Y\},\{x, y\}$ and $\left\{u_{X}, u_{Y}\right\}$, respectively. The element nodes are located at $\left\{X_{i}, Y_{i}\right\},(i=1, \ldots n)$ in the reference configuration $\mathcal{C}_{0}$ and move to $\left\{x_{i}=X_{i}+u_{X i}, y_{i}=Y_{i}+u_{Y_{i}}\right\},(i=1, \ldots n)$ in the current configuration $\mathcal{C}$. The element displacement field may be expressed as

$$
\left\{\begin{array}{l}
u_{X} \\
u_{Y}
\end{array}\right\}=\left[\begin{array}{ccccc}
N_{1} & 0 & N_{2} & \ldots & 0 \\
0 & N_{1} & 0 & \ldots & N_{n}
\end{array}\right]\left\{\begin{array}{c}
v_{X 1} \\
v_{Y 1} \\
v_{X 2} \\
\vdots \\
v_{Y n}
\end{array}\right\}=\mathbf{N} \mathbf{v}
$$

in which $N_{i}$ are appropriate isoparametric shape functions written in terms of natural coordinates such as $\xi$ and $\eta$ for quadrilaterals. The $\mathbf{G}$ matrix follows upon differentiation with respect to $X$ and $Y$, and all core equations transformed as per (6.3)-(6.4). For example, the physical tangent stiffness is

$$
\mathbf{K}=\int_{V_{0}} \mathbf{G}^{T}\left(\mathbf{S}_{M}+\mathbf{S}_{G}\right) \mathbf{G} d V
$$

where $S_{M}$ and $S_{G}$ are given by (5.13) and (5.14), respectively. As in the case of linear elements, (7.7) is most conveniently evaluated by numerical integration. Because several of the integrand matrices are sparse, in the interest of efficiency in the computer implementation the integrand may be symbolically evaluated through a computer algebra system such as Macsyma, Maple or Mathematica, and automatically converted to Fortran or C program statements before being encapsulated in the Gauss quadrature loop.

\section{THE GENERALIZED CCF}

As discussed in Section 2, the Generalized Core Congruential Formulation or GCCF is required when the relation between displacement gradients $\mathbf{g}$ and finite element degrees of freedom $\mathbf{v}$ is nonlinear. This complication occurs in elements with rotational freedoms, such as beams, plates and shells, if finite rotations are exactly treated.

Recall the expression (4.8) of the second variation $\delta^{2} \mathcal{U}$ of the internal energy density. This expression has the core tangent stiffness $\mathbf{S}$ as kernel of the quadratic form in $\delta \mathrm{g}$. The core internal force $\boldsymbol{\Phi}$ also appears in the inner product $\left(\delta^{2} \mathbf{g}\right)^{T} \mathbf{\Phi}$. This second term may either survive or drop out depending on the relation of $\mathbf{g}$ with the target physical or generalized 
coordinates (the latter term is explained below) chosen in the CCF transformation phase. In the case of the DCCF, this term drops out and

$$
\mathbf{S}=\mathbf{S}_{M}+\mathbf{S}_{G}
$$

is the tangent core stiffness, which forward transforms as per (6.3). This is the situation considered so far. But if that term survives two things happen. First, (8.1) is relabeled as

$$
\mathbf{S}=\mathbf{S}_{M}+\mathbf{S}_{G P}
$$

in which $S$ and $S_{G P}$ are called the principal core tangent stiffness and principal geometric stiffness, respectively. Second, transforming the term $\left(\delta^{2} \mathbf{g}\right)^{T} \boldsymbol{\Phi}$ to freedoms $\mathbf{v}$ produces a extra term in accordance with the schematics

$$
\mathbf{K}=\mathbf{K}_{M}+\mathbf{K}_{G P}+\mathbf{K}_{G C}, \quad \mathbf{S}_{M} \rightarrow \mathbf{K}_{M}, \quad \mathbf{S}_{G P} \rightarrow \mathbf{K}_{G P}, \quad\left(\delta^{2} \mathbf{g}\right)^{T} \mathbf{\Phi} \mapsto \delta \mathbf{v}^{T} \mathbf{K}_{G C} \delta \mathbf{v},
$$

where $\rightarrow$ and $\mapsto$ symbolize DCCF-transformation and GCCF-transformation-styles, respectively. As can be seen the transformation phase produces a new term $\mathbf{K}_{G C}$ called the complementary geometric stiffness. That term cannot be expressed in terms of the variation $\delta \mathbf{g}$ of the displacement gradients. Consequently there is no "core complementary core geometric stiffness" $\mathbf{S}_{G C}$ that can be added to (8.2). Instead it appears as a "carry forward term" that materializes as a quadratic-form kernel upon transforming.

\subsection{Generalized Coordinates as Generic Target}

For elements that require the GCCF treatment a one-shot transformation between $\mathbf{g}$ and $\mathbf{v}$ is often replaced by a multistage transformation. The degree of freedom sets used as intermediate targets of this process will be collectively referred to as "generalized coordinates" and identified as q. Of course the final target: element node displacements $\mathbf{v}$, is a particular instance of such array of choices.

In Section 2 it was noted that two variants of the GGCF, qualified as algebraic and differential, should be distinguished in terms of consequences on the existence of physical stiffness equations at various variational levels. These variants are examined below. The ensuing development examines the transformation from displacement gradients $\mathbf{g}$ to a "generic target" set of generalized coordinates $q_{i}$ collected in vector q. These coordinates are assumed to be independent, a restriction removed later. Symbols $\mathbf{K}$ and $\mathbf{f}$ are used to denote tangent stiffness matrices and internal force vectors, respectively, in terms of $\mathbf{q}$.

\subsection{Algebraic Transformation}

The.Algebraic GCCF, or AGCCF, applies if the relation between $\mathbf{g}$ (source) and $\mathbf{q}$ (target) is nonlinear but algebraic. We have $\mathbf{g}=\mathbf{g}(\mathbf{q})$ or in index notation, $g_{i}=g_{i}\left(q_{j}\right)$. Differentiating with respect to the $q_{i}$ variables yields

$$
\begin{aligned}
\delta g_{i} & =\frac{\partial g_{i}}{\partial q_{j}} \delta q_{j}=G_{i j} \delta q_{j}, \quad \text { or } \delta \mathbf{g}=\mathbf{G} \delta \mathbf{q}, \\
\delta^{2} g_{i} & =\frac{\partial^{2} g_{i}}{\partial q_{j} \partial q_{k}} \delta q_{j} \delta q_{k}+\frac{\partial g_{i}}{\partial q_{j}} \delta^{2} \not \nearrow_{j}^{0}=F_{i j k} \delta q_{j} \delta q_{k}, \quad \text { or } \delta^{2} \mathbf{g}=(\mathbf{F} \delta \mathbf{q}) \delta \mathbf{q}
\end{aligned}
$$


Here $(\mathbf{F} \delta \mathbf{q})$ is the matrix $F_{i j k} \delta q_{k}=F_{k i j} \delta q_{k} ; \mathbf{F}$ being a cubic array. The array $\mathbf{G}$ receives the name tangent transformation matrix. The second term in the expansion of $\delta^{2} g_{i}$ vanishes because the $q_{i}$ are assumed to be independent target variables.

Enforcing invariance of $\delta^{2} U$ yields the tangent stiffness transformation

$$
\mathbf{K}=\int_{V_{0}}\left\{\mathbf{G}^{T}\left(\mathbf{S}_{M}+\mathbf{S}_{G P}\right) \mathbf{G}+\mathbf{Q}\right\} d V_{0}=\mathbf{K}_{M}+\mathbf{K}_{G P}+\mathbf{K}_{G C}=\mathbf{K}_{M}+\mathbf{K}_{G},
$$

where the entries of $\mathbf{Q}$ are (cf. Remark 4.1) $Q_{i j}=Q_{j i}=F_{k i j} \Phi_{k}$ with summation on $k=1, \ldots n_{g}$. Note that $\mathbf{Q}$ is symmetric because $F_{k i j}=F_{k j i}$. Integration of $\mathbf{Q}$ over $V_{0}$ yields the complementary portion $\mathbf{K}_{G C}$ of the geometric stiffness $\mathbf{K}_{G}$.

The internal, applied and prestress force vectors transform according to the formulas in (6.4) with the $\mathbf{G}$ defined in (8.4):

$$
\mathbf{f}=\int_{V_{0}} \mathbf{G}^{T} \mathbf{\Phi} d V_{0}, \quad \mathbf{p}=\int_{V_{0}} \mathbf{G}^{T} \boldsymbol{\Psi} d V_{0}, \quad \mathbf{p}^{0}=\int_{V_{0}} \mathbf{G}^{T} \boldsymbol{\Psi}^{0} d V_{0} .
$$

What happens to $\mathbf{K}^{U}$ and $\mathbf{K}^{r}$ ? They can be obtained, somewhat artificially, by constructing the matrix equation

$$
\mathbf{g}=\mathbf{W} \mathbf{q}
$$

where $\mathbf{W}$ is called a secant transformation matrix. Generally this matrix is far from unique because its $n_{g} \times n_{q}$ entries must satisfy only $n_{g}$ conditions. (Care has often to be given to the $q_{j} \rightarrow 0$ if $0 / 0$ limits appear in W.) Using (8.7) we can proceed to form

$$
\mathbf{K}^{U}=\int_{V_{0}} \mathbf{W}^{T} \mathbf{S}^{U} \mathbf{W} d V_{0}, \quad \mathbf{K}^{r}=\int_{V_{0}} \mathbf{G}^{T} \mathbf{S}^{r} \mathbf{W} d V_{0} .
$$

Because in general $\mathbf{W} \neq \mathbf{G}$, symmetry in the secant stiffness $\mathbf{K}^{r}$ cannot be expected even if $\mathbf{S}^{r}$ is symmetric.

REMARK 8.1. The AGGCF is applicable to finite elements with degrees of freedoms that include fixed-axis rotations, because such rotations are integrable. Examples are provided by twodimensional beams as well as plane stress (membrane) elements with drilling freedoms if only in-plane motions are allowed.

REMARK 8.2. Why is $\mathbf{K}_{G C}$ called a geometric stiffness? Because it vanishes if the current configuration is stress free, in which case the core internal force $\mathbf{\Phi}$ vanishes and so does $\mathbf{Q}$.

\subsection{Differential Transformation}

The Differential GCCF, or DGCCF, is required if the relation between $\mathbf{g}$ (source) and $\mathbf{q}$ (target) is only available as a non-integrable differential form between their variations:

$$
\begin{aligned}
\delta g_{i} & =G_{i j} \delta q_{j}, \quad \text { or } \delta \mathbf{g}=\mathbf{G} \delta \mathbf{q}, \\
\delta^{2} g_{i} & =\frac{\partial G_{i j}}{\partial q_{k}} \delta q_{j} \delta q_{k}=F_{i j k} \delta q_{j} \delta q_{k}, \quad \text { or } \quad \delta^{2} \mathbf{g}=(\mathbf{F} \delta \mathbf{q}) \delta \mathbf{q} .
\end{aligned}
$$


The transformation equation (8.5) still applies for $\mathbf{K}$ whereas (8.6) holds for the force vectors. But no integral $\mathbf{g}=\mathbf{g}(\mathbf{q})$ as in the AGGCF exists. Consequently $\mathbf{K}^{U}$ and $\mathbf{K}^{r}$, which require a secant matrix relation of the form (8.7), cannot be constructed. Furthermore $\mathbf{Q}$ is not necessarily symmetric; a condition for that being $F_{k i j}=F_{k j i}$ or equivalently $\partial G_{k i} / \partial q_{j}=\partial G_{k j} / \partial q_{i}$.

REMARK 8.3. For mechanical finite elements the DGCCF naturally arises when threedimensional finite rotations are present as nodal degrees of freedom, because such rotations are non-integrable.

REMARK 8.4. The relations (8.9) have points of resemblance with the case of non-holonomic constraints in analytical dynamics.

\subsection{Multistage Transformation}

Up to this point the $\mathrm{q}$ have been assumed to be independent variables. But as previously noted, for complicated elements the GCCF transformations are more conveniently applied in stages. The target variables in one stage become the source variables for the next one.

What happens if the $q$ are intermediate variables in a transformation chain? If the $q$ are linear in the final independent degrees of freedom $\mathbf{v}$, all previous formulas hold because the DCCF applies for the remaining transformations, which are strictly congruential. But if the $\mathbf{q}$ are nonlinear in $\mathbf{v}$, or only a non-integrable differential relation exists, term $\left(\partial g_{i} / \partial q_{j}\right) \delta^{2} q_{j}=G_{i j} \delta^{2} q_{j}$ in the second of (8.4) survives. The net effect is that the geometric stiffness acquires a higher order component, implicitly defined as the kernel of

$$
\int_{V_{0}} \Phi_{i} G_{i j} \delta^{2} q_{j} d V_{0}
$$

This term cannot be resolved ("resolution" meaning explicit extraction of its stiffness kernel in the form of a complementary geometric stiffness) until the transformation chain reaches downstream variables that either are the final degrees of freedom (and thus independent), or depend linearly on such. It is difficult to state detailed rules that encompass all possible situations. Instead the treatment of the $2 \mathrm{D}$ and $3 \mathrm{D}$ beam element transformations in Sections 9-10 illustrates the basic techniques for "carrying forward" terms such as (8.10).

\section{A 2-NODE 2D TIMOSHENKO BEAM ELEMENT}

We continue here with the derivation of a $2 \mathrm{D}$, isotropic Timoshenko beam element started in Section 5.4. This example serves to illustrate the Algebraic GCCF. The specific element constructed here has two end nodes, six degrees of freedom, and reference length $L_{0}$. The cross section area $A \equiv A_{0}$ and moment of inertia $I=\int_{A} Y^{2} d A$ are constant along the element. Axis $X$ is made to pass through the centroid so that $\int_{A} Y d A=0$. Furthermore it is assumed that the cross section is doubly symmetric so that $\int_{A} Y^{3} d A=0$. 
The element displacement field, defined by $u_{0 X}(X), u_{0 Y}(X)$ and $\theta(X)$, is interpolated with linear shape functions:

$$
\left\{\begin{array}{c}
u_{0 X} \\
u_{0 Y} \\
\theta
\end{array}\right\}=\left[\begin{array}{cccccc}
N_{1} & 0 & 0 & N_{2} & 0 & 0 \\
0 & N_{1} & 0 & 0 & N_{2} & 0 \\
0 & 0 & N_{1} & 0 & 0 & N_{2}
\end{array}\right]\left\{\begin{array}{c}
v_{x 1} \\
v_{y 1} \\
\theta_{1} \\
v_{x 2} \\
v_{y 2} \\
\theta_{2}
\end{array}\right\}=N v
$$

where $N_{1}=1-\left(X / L_{0}\right)$ and $N_{2}=1-N_{1}=X / L_{0}$. Consequently

$$
\epsilon=\frac{\partial u_{0 X}}{\partial X}=\frac{v_{X 2}-v_{X 1}}{L_{0}}, \quad \gamma=\frac{\partial u_{0 Y}}{\partial X}=\frac{v_{X 2}-v_{X 1}}{L_{0}}, \quad \kappa=\frac{\partial \theta}{\partial X}=\frac{\theta_{2}-\theta_{1}}{L_{0}},
$$

are constant over the element.

\subsection{Generalized Coordinates and Stress Resultants}

As intermediate set of generalized coordinates we take $\mathbf{q}^{T}=\left[\begin{array}{llll}\epsilon & \gamma & \kappa & \theta\end{array}\right]$. These four quantities are constant over each cross section and may be viewed as cross-section orientation coordinates. Consequently when obtaining stiffness matrices and internal forces in terms of $\mathbf{q}$ it is convenient to integrate over the beam cross section. The resulting quantities appear naturally in terms of cross section stress-resultants as shown below. In terms of these generalized coordinates the auxiliary vectors $\mathbf{b}_{\boldsymbol{i}}$ listed in (5.28) become

$$
\mathbf{b}_{1}=\left\{\begin{array}{c}
1+g_{1} \\
g_{2} \\
0 \\
0
\end{array}\right\}=\left\{\begin{array}{c}
1+\epsilon-Y \kappa \cos \theta \\
\gamma-Y \kappa \sin \theta \\
0 \\
0
\end{array}\right\}, \quad \mathbf{b}_{2}=\left\{\begin{array}{c}
g_{3} \\
1+g_{4} \\
1+g_{1} \\
g_{2}
\end{array}\right\}=\left\{\begin{array}{c}
-\sin \theta \\
\cos \theta \\
1+\epsilon-Y \kappa \cos \theta \\
\gamma-Y \kappa \sin \theta
\end{array}\right\}
$$

The well known stress resultants of beam theory are the axial force $N$, transverse shear force $V$ and bending moment $M$. They are obtained by integrating the PK2 stresses over the beam cross section:

$$
\begin{aligned}
N & =\int_{A_{0}} s_{1} d A=E A\left(\epsilon+\frac{1}{2}\left(\epsilon^{2}+\gamma^{2}\right)\right)+\frac{1}{2} E I \kappa^{2}+N^{0}, \\
V & =\int_{A_{0}} s_{2} d A=G A_{s} \omega_{\gamma}+V^{0}, \\
M & =\int_{A_{0}} s_{1} Y d A=-E I \kappa \omega_{\epsilon}+M^{0},
\end{aligned}
$$

where $\omega_{\epsilon}=(1+\epsilon) \cos \theta+\gamma \sin \theta$ and $\omega_{\gamma}=\gamma \cos \theta-(1+\epsilon) \sin \theta$ can be viewed as generalized skew strains. In (9.4) $N^{0}, V^{0}$ and $M^{0}$ denote initial-stress resultants (stress 
resultants in $\mathcal{C}_{0}$, also called prestress forces), $A \equiv A_{0}, I=\int_{A_{0}} Y^{2} d A$, and $A_{s}=\mu A$, in which $\mu$ is the usual shear correction factor of Timoshenko beam theory. Because of the doubly-symmetric cross-section assumption, a term containing the third-section-moment $\int_{A_{0}} Y^{3} d A$ has been omitted from the expression for $M$.

In addition to $N, V$ and $M$, the following higher order moment, which is absent from the linear theory, appears in the residual force and tangent stiffness:

$$
C=\int_{A_{0}} s_{1} Y^{2} d A=E I\left(\left(\epsilon+\frac{1}{2}\left(\epsilon^{2}+\gamma^{2}\right)\right)+\frac{1}{2} E \mathcal{H} \kappa^{2}\right)+C^{0}
$$

in which $\mathcal{H}=\int_{A_{0}} Y^{4} d A$. If terms in $\kappa^{2}$ are neglected,

$$
C-C^{0}=\left(N-N^{0}\right)(I / A)=\left(N-N^{0}\right) r^{2},
$$

where $r=\sqrt{I / A}$ is the radius of gyration of the cross section. If such terms are retained this relation is only exact if $r^{2}=\mathcal{H} / I$ and approximate otherwise.

REMARK 9.1. One may verify that $\int_{A} s_{2} Y d A$ vanishes identically. This serves as a check of the strain distribution equations.

\subsection{Transformation Matrices}

The differential relations required to establish the tangent transformation are obtained from (5.25) as

$$
\begin{gathered}
\delta \mathbf{g}=\frac{\partial \mathbf{g}}{\partial \mathbf{q}} \delta \mathbf{q}=\left[\begin{array}{cccc}
1 & 0 & -Y \cos \theta & Y \kappa \sin \theta \\
0 & 1 & -Y \sin \theta & -Y \kappa \cos \theta \\
0 & 0 & 0 & -\cos \theta \\
0 & 0 & 0 & -\sin \theta
\end{array}\right]\left\{\begin{array}{c}
\delta \epsilon \\
\delta \gamma \\
\delta \kappa \\
\delta \theta
\end{array}\right\}=\mathbf{G}_{1} \delta \mathbf{q} \\
\delta \mathbf{q}=\frac{\partial \mathbf{q}}{\partial \mathbf{v}} \delta \mathbf{v}=\frac{1}{L_{0}}\left[\begin{array}{cccccc}
-1 & 0 & 0 & 1 & 0 & 0 \\
0 & -1 & 0 & 0 & 1 & 0 \\
0 & 0 & -1 & 0 & 0 & 1 \\
0 & 0 & L_{0}-X & 0 & 0 & X
\end{array}\right]\left\{\begin{array}{c}
\delta v_{X 1} \\
\delta v_{Y 1} \\
\vdots \\
\delta \theta_{2}
\end{array}\right\}=\mathbf{G}_{2} \delta \mathbf{v}
\end{gathered}
$$

The transformation relating $\delta \mathbf{g}=\mathbf{G} \delta \mathbf{v}$ may be obtained as the product

$$
\mathbf{G}=\mathbf{G}_{1} \mathbf{G}_{2}=\frac{1}{L_{0}}\left[\begin{array}{cccccc}
-1 & 0 & Y\left(\cos \theta+\left(L_{0}-X\right) \kappa \sin \theta\right) & 1 & 0 & Y(-\cos \theta+X \kappa \sin \theta) \\
0 & -1 & Y\left(\sin \theta-\left(L_{0}-X\right) \kappa \cos \theta\right) & 0 & 1 & Y(-\sin \theta-X \kappa \cos \theta) \\
0 & 0 & -\left(L_{0}-X\right) \cos \theta & 0 & 0 & -X \cos \theta \\
0 & 0 & -\left(L_{0}-X\right) \sin \theta & 0 & 0 & -X \sin \theta
\end{array}\right]
$$

but it is more instructive (as well as conducive to higher efficiency in the computer implementation) to perform the transformation phase in two stages.

Observe that the first transformation (from $g$ to $q$ ) is nonlinear and algebraic whereas the second one (from $\mathbf{q}$ to $\mathbf{v}$ ) is linear. Consequently we have to use the AGCCF for the first transformation but the second one can be done simply through the DCCF. 


\subsection{Internal Force Vector}

The internal force vector in terms of $\mathbf{q}$, denoted by $\mathbf{f}^{q}$, is obtained from the core expression (5.32) for $\boldsymbol{\Phi}$ and the matrix $\mathbf{G}_{1}$ given in (9.7):

$$
\mathbf{f}^{q}=\int_{A_{0}} \mathbf{G}_{1}^{T} \Phi d A_{0}=\left\{\begin{array}{l}
f_{\epsilon}^{q} \\
f_{\gamma}^{q} \\
f^{q} \\
f_{\theta}^{q}
\end{array}\right\}=\left\{\begin{array}{c}
N(1+\epsilon)-M \kappa \cos \theta-V \sin \theta \\
N \gamma-M \kappa \sin \theta+V \cos \theta \\
-M \omega_{\epsilon}+C \kappa \\
-M \kappa \omega_{\gamma}-V \omega_{\epsilon}
\end{array}\right\} .
$$

Finally, application of (9.8) and integration over the element length yields

$$
\mathbf{f}=\int_{0}^{L_{0}} \mathbf{G}_{2}^{T} \mathbf{f}^{q} d X=\left\{\begin{array}{c}
-f_{\epsilon}^{q} \\
-f_{\gamma}^{q} \\
-f_{\kappa}^{q}+\frac{1}{2} L_{0} f_{\theta}^{q} \\
f_{\epsilon}^{q} \\
f_{\gamma}^{q} \\
f_{\kappa}^{q}+\frac{1}{2} L_{0} f_{\theta}^{q}
\end{array}\right\}
$$

This vector satisfies translational equilibrium.

\subsection{Tangent Stiffness Matrix}

Transforming to generalized coordinates q produces three components of the tangent stiffness matrix:

$$
\mathbf{K}^{q}=\int_{A_{0}}\left(\mathbf{G}_{1}^{T}\left(\mathbf{S}_{M}+\mathbf{S}_{G}\right) \mathbf{G}_{1}+\mathbf{Q}\right) d A=\mathbf{K}_{M}^{q}+\mathbf{K}_{G P}^{q}+\mathbf{K}_{G C}^{q} .
$$

The entries of $\mathbf{K}_{M}^{q}$, obtained through symbolic manipulation, are

$$
\begin{aligned}
& K_{M}^{q}(1,1)=E A(1+\epsilon)^{2}+G A_{s} \sin ^{2} \theta+E I \kappa^{2} \cos ^{2} \theta, \\
& K_{M}^{q}(1,2)=E A(1+\epsilon) \gamma-G A_{s} \sin \theta \cos \theta+E I \kappa^{2} \sin \theta \cos \theta, \\
& K_{M}^{q}(1,3)=E I \kappa\left((1+\epsilon)\left(1+\cos ^{2} \theta\right)+\gamma \sin \theta \cos \theta\right), \\
& K_{M}^{q}(1,4)=E I \kappa^{2} \omega_{\gamma} \cos \theta+G A_{s} \omega_{\epsilon} \sin \theta \\
& K_{M}^{q}(2,2)=E A \gamma^{2}+G A_{s} \cos ^{2} \theta+E I \kappa^{2} \sin ^{2} \theta \\
& K_{M}^{q}(2,3)=E I \kappa\left((1+\epsilon) \sin \theta \cos \theta+\gamma\left(1+\sin ^{2} \theta\right)\right), \\
& K_{M}^{q}(2,4)=E I \kappa^{2} \omega_{\gamma} \sin \theta-G A_{s} \omega_{\epsilon} \cos \theta \\
& K_{M}^{q}(3,3)=E I \omega_{\epsilon}^{2}+E \mathcal{H} \kappa^{2}, \\
& K_{M}^{q}(3,4)=E I \kappa\left((1+\epsilon) \gamma\left(\cos ^{2} \theta-\sin ^{2} \theta\right)+\left(\gamma^{2}-(1+\epsilon)^{2}\right) \sin \theta \cos \theta\right), \\
& K_{M}^{q}(4,4)=E I \kappa^{2} \phi_{g}^{2}+G A_{s} \omega_{\epsilon}{ }^{2} .
\end{aligned}
$$

The principal geometric stiffness, which is readily worked out by hand, is 


$$
\mathbf{K}_{G P}^{q}=\left[\begin{array}{cccc}
N & 0 & -M \cos \theta & M \kappa \sin \theta-V \cos \theta \\
& N & -M \sin \theta & -M \kappa \cos \theta-V \sin \theta \\
& & C & 0 \\
\text { symm } & & & C \kappa^{2}
\end{array}\right]
$$

The new term contributed by the AGCCF to $\mathrm{K}^{q}$ is the complementary geometric stiffness $\mathbf{K}_{G C}^{q}$. Its source is the matrix $\mathbf{Q}$ introduced in Section 8.2. The entries of $\mathbf{Q}$ are $Q_{i j}=$ $\left(\partial^{2} g_{k} / \partial q_{i} \partial q_{j}\right) \Phi_{k}$, where the components of $\mathbf{g}$ and $\boldsymbol{\Phi}=s_{1} \mathbf{b}_{1}+s_{2} \mathbf{b}_{2}$ may be obtained from (5.25) and (9.3), respectively.

The entries of $\mathbf{Q}$ were symbolically generated by the following Mathematica module:

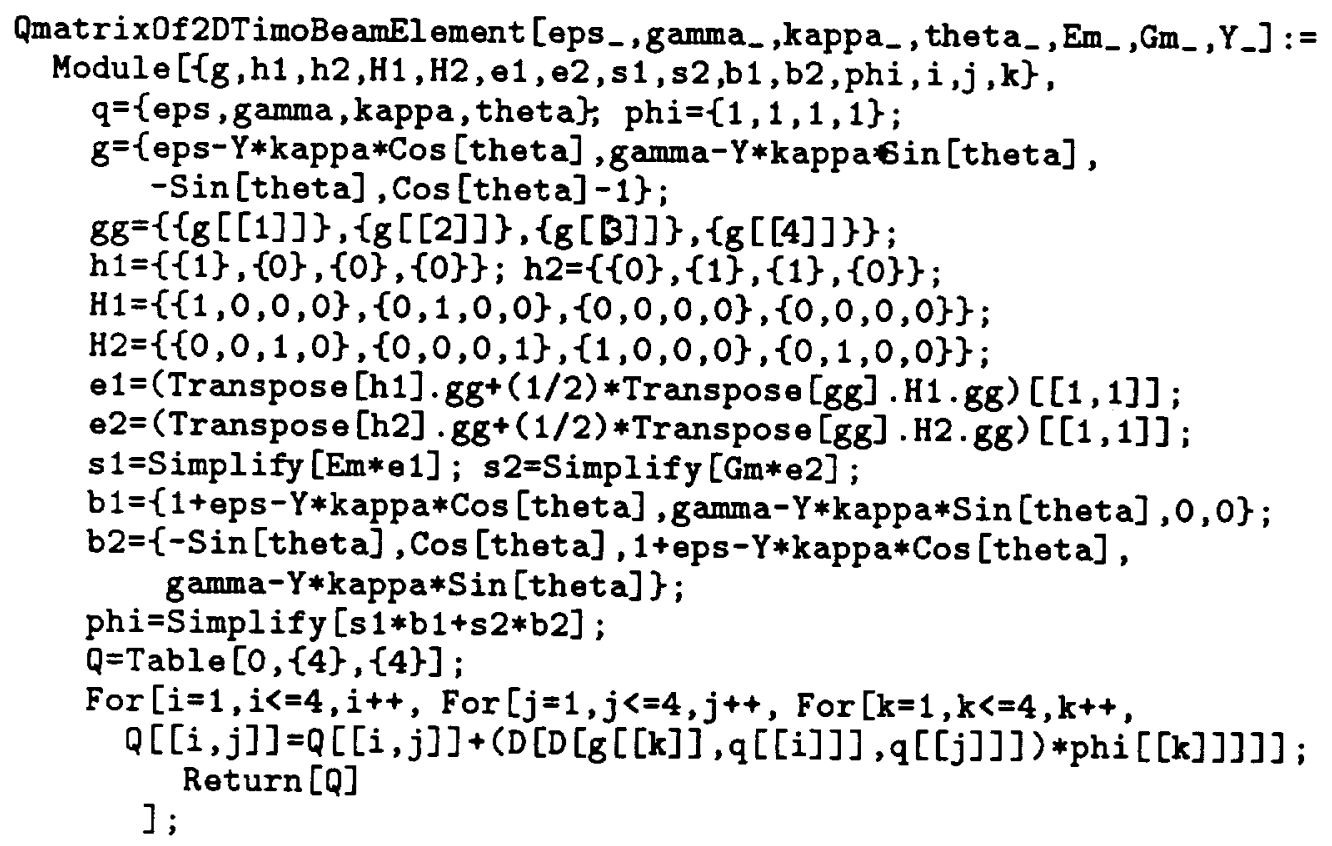

The output of this module was integrated over the cross section and pattern matched with the expression of the stress resultants (9.4)-(9.5) to produce

$$
\mathbf{K}_{G C}^{q}=\left[\begin{array}{cccc}
0 & 0 & 0 & 0 \\
& 0 & 0 & 0 \\
& & 0 & -M \omega_{\gamma} \\
s y m m & & & -V \omega_{\gamma}+M \kappa \omega_{\epsilon}-C \kappa^{2}
\end{array}\right],
$$

which added to (9.14) yields the geometric stiffness

$$
\mathbf{K}_{G}^{q}=\left[\begin{array}{cccc}
N & 0 & -M \cos \theta & M \kappa \sin \theta-V \cos \theta \\
& N & -M \sin \theta & -M \kappa \cos \theta-V \sin \theta \\
& & C & -M \omega_{\gamma} \\
\text { symm } & & & -V \omega_{\gamma}+M \kappa \omega_{\epsilon}
\end{array}\right] .
$$


Finally, the tangent stiffness in terms of $q$ is $K^{q}=K_{M}+K_{G}$. Denoting the entries of $K^{q}$ by $K_{i j}^{q}, i, j=1, \ldots 4$ the tangent stiffness matrix $\mathbf{K}$ in terms of node displacements $\mathbf{v}$ is formed through the DCCF transformation

$$
\mathbf{K}=\int_{0}^{L_{0}} \mathbf{G}_{2}^{T} \mathbf{K}^{q} \mathbf{G}_{2} d X=\left[\begin{array}{ccc}
K_{11}^{q} & K_{12}^{q} & K_{13}^{q}-\frac{1}{2} L_{0} K_{14}^{q} \\
& K_{22}^{q} & K_{23}^{q}-\frac{1}{2} L_{0} K_{24}^{q} \\
& & K_{33}^{q}-L_{0} K_{34}^{q}+\frac{1}{3} L_{0}^{2} K_{44}^{q} \\
& \\
& & \\
& & \\
-K_{11}^{q} & -K_{12}^{q} & -K_{13}^{q}-\frac{1}{2} L_{0} K_{14}^{q} \\
-K_{12}^{q} & -K_{22}^{q} & -K_{23}^{q}-\frac{1}{2} L_{0} K_{24}^{q} \\
-K_{13}^{q}+\frac{1}{2} L_{0} K_{14}^{q} & -K_{23}^{q}+\frac{1}{2} L_{0} K_{24}^{q} & -K_{33}^{q}+\frac{1}{6} L_{0}^{2} K_{44}^{q} \\
K_{11}^{q} & K_{12}^{q} & K_{13}^{q}+\frac{1}{2} L_{0} K_{14}^{q} \\
& K_{22}^{q} & K_{23}^{q}+\frac{1}{2} L_{0} K_{24}^{q} \\
& & K_{33}^{q}+L_{0} K_{34}^{q}+\frac{1}{3} L_{0}^{2} K_{44}^{q}
\end{array}\right]
$$

The above rule can be applied to $\mathbf{K}_{M}$ and $\mathbf{K}_{G}$ should separate formation be desirable, as when setting up a stability eigenproblem. Using these schemes $K$ can be formed at a cost of approximately 300 floating-point operations per element, which is not too different from the cost of a TL 3D bar.

If the reference configuration is not aligned with $X$, the preceding expressions apply to the local system $\{\bar{X}, \bar{Y}\}$. A final local-to-global transformation step, similar to that discussed for the 3D bar in Section 7.1, is then necessary. This step can be handled by a simple DCCF transformation, because the finite rotation $\theta$ remains the same in global coordinates.

REMARK 9.2. The foregoing exact expressions contain curvature-squared terms typically in the combination $I \kappa^{2}$. This can be shown to be of order $(r / R)^{2}$ compared to other terms, where $r$ is the radius of gyration of the cross section and $R=1 / \kappa$ the radius of curvature of the current configuration. For typical beams $(r / R)^{2}$ is $10^{-6}$ or less; consequently all such tiny terms may be dropped without visible loss of accuracy. For highly-bent extremely-thin beams, however, that ratio may go up to 0.01 in which case the $\kappa^{2}$ terms might have a noticeable though small effect if retained.

\subsection{Can a Secant Stiffness be Constructed?}

To attempt the construction of a secant stiffness $\mathbf{K}^{r q}$ in terms of generalized coordinates $\mathbf{q}$ one should obtain a secant matrix form of the relationship $\mathbf{g}=\mathbf{g}(\mathbf{q})$. As noted in Section 8.2 , such form is far from unique. One possible choice is

$$
\mathbf{g}=\left\{\begin{array}{l}
g_{1} \\
g_{2} \\
\dot{g}_{2} \\
g_{4}
\end{array}\right\}=\left[\begin{array}{cccc}
1 & 0 & -Y \cos \theta & 0 \\
0 & 1 & -Y \sin \theta & 0 \\
0 & 0 & 0 & -\sin \theta / \theta \\
0 & 0 & 0 & (\cos \theta-1) / \theta
\end{array}\right]\left\{\begin{array}{l}
\epsilon \\
\gamma \\
\kappa \\
\theta
\end{array}\right\}=\mathbf{W}_{1} \mathbf{q},
$$


Table 1. Internal energy and its variations for 3D Timoshenko beam element

\begin{tabular}{|c|c|c|c|}
\hline $\begin{array}{c}\text { Core } \\
\text { Particle } \\
\mathbf{g}\end{array}$ & $\begin{array}{c}\text { Section Gradients } \\
\text { Cross-Section } \\
\mathbf{w}\end{array}$ & $\begin{array}{c}\text { Section Orientation } \\
\text { Cross-Section } \\
\mathbf{z}\end{array}$ & $\begin{array}{c}\text { Physical DOF } \\
\text { Whole Element } \\
\mathbf{v}\end{array}$ \\
\hline $\mathcal{U}=\frac{1}{2} \mathbf{g}^{T} \mathbf{S}^{U} \mathbf{g}+\mathbf{g}^{T} \mathbf{\Psi}^{0}$ & - & - & - \\
$\delta \mathcal{U}=\delta \mathbf{g}^{T}\left(\mathbf{S}^{r} \mathbf{g}+\mathbf{\Psi}^{0}\right)$ & $\delta U_{G}=\delta \mathbf{w}^{T} \mathcal{R}$ & $\delta U_{z}=\delta \mathbf{z}^{T} \mathbf{f}_{z}$ & $\delta U=\delta \mathbf{v}^{T} \mathbf{f}$ \\
$\delta^{2} \mathcal{U}=\delta \mathbf{g}^{T} \mathbf{S} \delta \mathbf{g}+\delta^{2} \mathbf{g}^{T} \mathbf{\Phi}$ & $\delta^{2} U_{G}=\delta \mathbf{w}^{T} \mathcal{S} \delta \mathbf{w}+\mathcal{F}$ & $\delta^{2} U_{z}=\delta \mathbf{z}^{T} \mathbf{K}_{z} \delta \mathbf{z}$ & $\delta^{2} U=\delta \mathbf{v}^{T} \mathbf{K} \delta \mathbf{v}$ \\
\hline
\end{tabular}

which has the merit of not being too dissimilar from $\mathbf{G}_{1}$. Note that some care must be taken as regards some $0 / 0$ limits. Then $\mathbf{K}^{r q}=\int_{A} \mathbf{G}_{1}^{T} \mathbf{S}^{r} \mathbf{W}_{1} d A$, which may be easily worked out in closed form but is unsymmetric. Because $\mathbf{q}$ is linear in $\mathbf{v}$, the next transformation is simply $\mathbf{K}^{r}=\int_{0}^{L_{0}} \mathbf{G}_{2}^{T} \mathbf{K}^{r q} \mathbf{G}_{2} d X$ which can be handled through a scheme similar to (9.17) but with an unsymmetric kernel matrix.

\section{A 2-NODE 3D TIMOSHENKO BEAM ELEMENT}

We continue here the development of a two-node 3D Timoshenko beam element started in Section 5.5. As can be surmised, the development is more complex and demanding than for its 2D counterpart. Only a summary taken from Crivelli's thesis ${ }^{5}$ and Crivelli and Felippa $^{7}$ is presented here. The transformation phase to pass from the core equations to the element nodal degrees of freedom is carried out in three stages:

1. From particle displacement gradients $\mathbf{g}$ to generalized gradients $\mathbf{w}$ at each cross section. An integration over the cross section area is involved.

2. From generalized gradients $\mathbf{w}$ to cross-section orientation coordinates $\mathbf{q}$. The rotational parametrization is introduced at this stage.

3. From cross-section orientation to finite-element nodal degrees of freedom $\mathbf{v}$. An integration over the element length, as defined by the shape functions, is involved.

These transformation stages are summarized in Tables 1 and 2, which together also serve to define notation

\subsection{Transformation to Generalized Gradients}

The first set of target variables are the generalized gradients $\mathbf{w}(X)$ at each reference cross section defined by the longitudinal coordinate $X$. The components of $\mathbf{w}$ are indirectly 
Table 2. Core-to-physical-DOFs transformations for 3D beam element

\begin{tabular}{|c|c|c|c|}
\hline $\begin{array}{c}\text { Core Level } \\
\text { Particle } \\
\text { g }\end{array}$ & $\begin{array}{l}\text { Section Gradients } \\
\text { Cross-Section } \\
\mathbf{w}\end{array}$ & $\begin{array}{l}\text { Section Orientation } \\
\text { Cross-Section } \\
\mathbf{z}\end{array}$ & $\begin{array}{c}\text { Physical DOF } \\
\text { Whole Element } \\
\mathbf{v}\end{array}$ \\
\hline$\Phi$ & $\mathcal{R}=\int_{A_{0}} \mathbf{W}^{T} \boldsymbol{\Phi} d A$ & $\mathbf{f}_{z}=\mathbf{Z}^{T} \mathcal{R}$ & $\mathbf{f}=\int_{0}^{L_{0}} \mathbf{G}_{z}^{T_{z}} \mathbf{f}_{z} d X$ \\
\hline $\mathbf{S}$ & $\mathcal{S}=\int_{A_{0}} \mathbf{W}^{T} \mathbf{S W} d A$ & $\mathbf{K}_{z}=\mathbf{Z}^{T} \mathcal{S} \mathbf{Z}+\mathcal{S}_{G C z}$ & $\mathbf{K}=\int_{0}^{L_{0}} \mathbf{G}_{z}^{T} \mathbf{K}_{z} \mathbf{G}_{z} d X$ \\
\hline \multicolumn{2}{|r|}{$N \delta \mathbf{w}$} & \multicolumn{2}{|c|}{$\delta \mathbf{z}=\mathbf{G}_{z} \delta \mathbf{v}$} \\
\hline
\end{tabular}

given through their first variation:

$$
\delta \mathbf{w}=\left\{\begin{array}{lll}
\frac{d \delta \mathbf{u}_{0}}{d X} & \frac{d \delta \Theta}{d X} & \delta \Theta
\end{array}\right\}^{T}
$$

where $\delta \Theta$, defined in (5.36), measures the variation of angular orientation. Because this quantity is not generally integrable for three-dimensional motions, it is not possible to express $\Theta$ as a unique function of the displacements. The variation of $\mathrm{g}_{1}$ is

$$
\delta \mathbf{g}_{1}=\frac{d \delta \mathbf{u}_{0}}{d X}+\mathbf{R}^{T} \tilde{\boldsymbol{\zeta}}^{T} \frac{d \delta \boldsymbol{\Theta}}{d X}+\mathbf{R}^{T} \tilde{\boldsymbol{\zeta}}^{T} \tilde{\boldsymbol{\kappa}} \delta \boldsymbol{\Theta}+\mathbf{R}^{T} \widetilde{\delta \Theta} \tilde{\boldsymbol{\zeta}}^{T} \boldsymbol{\kappa},
$$

where we used the relation ${ }^{5} \delta \boldsymbol{\kappa}=d \delta \Theta / d X+\tilde{\boldsymbol{\kappa}} \delta \Theta$. On using the commutative law ãb $=$ $\tilde{\mathbf{b}}^{T} \mathbf{a}$ and Jacobi's identity $\widetilde{\mathbf{a} b}=\tilde{\mathbf{a}} \tilde{\mathbf{b}}-\tilde{\mathbf{b}} \mathbf{a}$ we may rewrite (10.2) as

$$
\delta \mathbf{g}_{1}=\frac{\partial \delta \mathbf{u}_{0}}{\partial X}+\mathbf{R}^{T} \tilde{\boldsymbol{\zeta}}^{T} \frac{\partial \delta \boldsymbol{\Theta}}{\partial X}+\mathbf{R}^{T} \tilde{\boldsymbol{\kappa}}^{T} \tilde{\boldsymbol{\zeta}} \delta \boldsymbol{\Theta}
$$

For the other gradient vectors we have $\delta \mathbf{g}_{2}=\delta \mathbf{R}^{T} \mathbf{h}_{2}=\mathbf{R}^{T} \widetilde{\delta \Theta} \mathbf{h}_{2}=\mathbf{R}^{T} \tilde{\mathbf{h}}_{2}^{T} \delta \Theta$ and $\delta \mathbf{g}_{3}=$ $\mathbf{R}^{T} \tilde{\mathbf{h}}_{3}^{T} \delta \Theta$, which can be collected in matrix form as

$$
\delta \mathbf{g}=\left\{\begin{array}{l}
\delta \mathbf{g}_{1} \\
\delta \mathbf{g}_{2} \\
\delta \mathbf{g}_{3}
\end{array}\right\}=\left[\begin{array}{ccc}
\mathbf{I} & \mathbf{R}^{T} \tilde{\boldsymbol{\zeta}}^{T} & \mathbf{R}^{T} \tilde{\boldsymbol{\kappa}}^{T} \tilde{\boldsymbol{\zeta}} \\
0 & 0 & \mathbf{R}^{T} \tilde{\mathbf{h}}_{2}^{T} \\
0 & 0 & \mathbf{R}^{T} \tilde{\mathbf{h}}_{3}^{T}
\end{array}\right]\left\{\begin{array}{c}
\frac{d \delta \mathbf{u}_{0}}{d X} \\
\frac{d \delta \boldsymbol{\Theta}}{d X} \\
\delta \boldsymbol{\Theta}
\end{array}\right\}=\left[\begin{array}{c}
\mathbf{W}_{1} \\
\mathbf{W}_{2} \\
\mathbf{W}_{3}
\end{array}\right] \delta \mathbf{w}=\mathbf{W} \delta \mathbf{w}
$$

where $I$ is the 3 -by-3 identity matrix and $W_{i}$ are 3 -by- 9 matrices. The second variation of $\mathbf{g}$, which is required for the complementary geometric stiffness, is

$$
\begin{aligned}
\delta^{2} \mathbf{g}_{1}= & \mathbf{R}^{T} \widetilde{\delta} \tilde{\zeta}^{T} \frac{d \delta \boldsymbol{\Theta}}{d X}+\mathbf{R}^{T} \widetilde{\delta \Theta} \tilde{\boldsymbol{\zeta}}^{T} \tilde{\boldsymbol{\kappa}} \delta \boldsymbol{\Theta}+\mathbf{R}^{T} \widetilde{\delta \Theta} \tilde{\boldsymbol{\zeta}}^{T} \frac{d \delta \boldsymbol{\Theta}}{d X} \\
& +\mathbf{R}^{T} \widetilde{\delta \boldsymbol{\Theta}} \tilde{\boldsymbol{\zeta}}^{T} \tilde{\boldsymbol{\kappa}} \delta \boldsymbol{\Theta}+\delta^{2} \mathbf{R}^{T} \tilde{\boldsymbol{\zeta}}^{T} \boldsymbol{\kappa}+\mathbf{R}^{T} \tilde{\boldsymbol{\zeta}}^{T} \delta^{2} \boldsymbol{\kappa}, \\
\delta^{2} \mathbf{g}_{2}= & \delta^{2} \mathbf{R}^{T} \mathbf{i}_{2}, \quad \delta^{2} \mathbf{g}_{3}=\delta^{2} \mathbf{R}^{T} \mathbf{i}_{3}
\end{aligned}
$$


At this point it is appropriate to introduce the following section resultants:

$$
\begin{array}{rlrl}
\mathcal{P} & =A \sigma_{b}+\mathcal{P}^{0}, & s_{b}=E e_{b}, & \\
\mathcal{Q} & =\mu_{s} A+\mathcal{Q}^{0}, & \tau=\tau_{2}+\tau_{3}, \quad \tau_{2}=G \gamma_{2} \mathbf{h}_{2}, \quad \tau_{3}=G \gamma_{3} \mathbf{h}_{3}, \\
\mathcal{M}_{\sigma} & =E \mathbf{I}_{S} \mathcal{K}_{e}+\mathcal{M}_{\sigma}^{0}, & \mathbf{I}_{S}=\int_{A_{0}} \zeta \zeta^{T} d A, \quad \mathcal{K}_{e}=\tilde{\phi} \kappa, \\
\mathcal{M}_{\tau} & =\mu_{t} G \mathbf{I}_{P} \kappa+\mathcal{M}_{\tau}^{0}, & \mathbf{I}_{P}=\int_{A_{0}} \tilde{\zeta} \tilde{\zeta}^{T} d A .
\end{array}
$$

Here $\mathcal{P}, \mathcal{Q}, \mathcal{M}_{\sigma}$ and $\mathcal{M}_{\tau}$ are axial forces, shear forces, bending moments and torsional moments, respectively, at the current configuration $\mathcal{C} ; \mathcal{P}^{0}, \mathcal{Q}^{0}, \mathcal{M}_{\sigma}^{0}$ and $\mathcal{M}_{\tau}^{0}$ are similar quantities at the reference configuration $\mathcal{C}_{0} ; \mu_{s}$ and $\mu_{t}$ are transverse-shear and torsion coefficients that account for the actual shear stress distributions, respectively; and $\mathbf{I}_{S}$ and $\mathbf{I}_{P}$ are the cartesian and polar inertia tensors, respectively, of the cross section. Should the axes $Y$ and $Z$ be aligned with the principal inertia axes the latter simplified to

$$
\mathbf{I}_{S}=\left[\begin{array}{ccc}
0 & 0 & 0 \\
0 & I_{22} & 0 \\
0 & 0 & I_{33}
\end{array}\right], \quad \mathbf{I}_{P}=\left[\begin{array}{ccc}
I_{22}+I_{33} & 0 & 0 \\
0 & I_{33} & 0 \\
0 & 0 & I_{22}
\end{array}\right]
$$

Because the relation between $\mathbf{g}$ and $\mathbf{w}$ is of differential type the applicable transformation rules are those the DGCCF, and no energy or secant stiffness survives. Thus only the internal force vector $\mathcal{R}$ and tangent stiffness $\mathcal{S}$ associated with $\mathbf{w}$ are derived below.

Internal Force Vector. The generalized internal force vector is

$$
\mathcal{R}=\int_{A_{0}} \mathbf{W}^{T} \boldsymbol{\Phi} d A=\sum_{i} \int_{A_{0}} s_{i} \mathbf{W}^{T} \mathbf{b}_{i} d A=\mathcal{R}_{\sigma}+\mathcal{R}_{\tau}
$$

where $\mathcal{R}_{\sigma}$ and $\mathcal{R}_{\tau}$ are the contributions of the normal and shear stresses respectively. Detailed calculations result ${ }^{5}$ in the following exact expressions:

$$
\mathcal{R}_{\sigma}=\left[\begin{array}{c}
\mathbf{R}^{T}\left(\mathcal{P} \phi+\tilde{\boldsymbol{\kappa}} \mathcal{M}_{\sigma}\right) \\
\tilde{\phi}^{T} \mathcal{M}_{\sigma} \\
\tilde{\mathcal{K}}_{e}^{T} \mathcal{M}_{\sigma}
\end{array}\right], \quad \mathcal{R}_{\tau}=\left[\begin{array}{c}
\mathbf{R}^{T} \mathcal{Q} \\
\mathcal{M}_{\tau} \\
\tilde{\phi}^{T} \mathcal{Q}+\tilde{\boldsymbol{\kappa}}^{T} \mathcal{M}_{\tau}
\end{array}\right]
$$

For small deformations in which the squared curvature may be neglected, $\mathbf{R} \approx \mathbf{I}, \tilde{\phi} \approx \widetilde{\mathbf{h}}_{\mathbf{1}}$, $\mathcal{K}_{e} \approx \kappa$ and $\tilde{\kappa} \mathcal{M}_{\sigma} \approx 0$. If these approximations are made,

$$
\mathcal{R}_{\sigma}=\left[\begin{array}{c}
\mathcal{P} \mathbf{h}_{1} \\
\widetilde{\mathbf{h}}_{1}^{T} \mathcal{M}_{\sigma} \\
0
\end{array}\right], \quad \mathcal{R}_{\tau}=\left[\begin{array}{c}
\mathcal{Q} \\
\mathcal{M}_{\tau} \\
\widetilde{\mathbf{h}}_{1}^{T} \mathcal{Q}
\end{array}\right]
$$


These resemble the classic linearized theory equations. Furthermore observe that the term $\mathcal{P} \mathbf{R}^{T} \phi$ corresponds to the internal force of the TL 3D bar.

Tangent Stiffness. For the tangent stiffness we have the decomposition

$$
\mathcal{S}=\mathcal{S}_{M}+\mathcal{S}_{G P}+\mathcal{S}_{G C}
$$

Furthermore, since $\mathbf{w}$ is nonlinear in downstream variables, the complementary geometric stiffness splits into two components:

$$
\mathcal{S}_{G C}=\mathcal{S}_{G C \boldsymbol{w}}+\mathcal{S}_{G C q}
$$

where $\mathcal{S}_{G C w}$ and $\mathcal{S}_{G C q}$ contains terms that depend on the first and second variations, respectively, of $\mathbf{R}$ and $\kappa$. The notation is suggested by the fact that $\mathcal{S}_{G C w}$ can be merged into $\mathcal{S}_{G P}$ to yield the geometric stiffness $\mathcal{S}_{G w}=\mathcal{S}_{G P}+\mathcal{S}_{G C w}$, which is associated with the generalized gradients $\mathbf{w}$ and independent of the rotational parametrization selected in the next set of target variables $q$. On the other hand, the kernel $\mathcal{S}_{G C q}$ cannot be extracted at the $\mathbf{w}$ level and must be carried forward to the $q$ level because it is parametrization dependent. Each of the components in (10.11)-(10.12) may be expressed as the sum of two contributions, one from the normal stresses and one from the shear stresses:

$$
\mathcal{S}_{M}=\mathcal{S}_{M \sigma}+\mathcal{S}_{M \tau}, \quad \mathcal{S}_{G P}=\mathcal{S}_{G P \sigma}+\mathcal{S}_{G P \tau}, \quad \mathcal{S}_{G C x}=\mathcal{S}_{G C x \sigma}+\mathcal{S}_{G C x \tau}, \quad x=w, q
$$

Material Stiffness. The generalized core material stiffness is given by the congruential transformation

$$
\mathcal{S}_{M}=\int_{A_{0}} \mathbf{W}^{T} \mathbf{S}_{M} \mathbf{W} d A=\sum_{i} \int_{A_{0}} E_{i} \mathbf{W}^{T} \mathbf{b}_{i} \mathbf{b}_{i}^{T} \mathbf{W} d A=\mathcal{S}_{M \sigma}+\mathcal{S}_{M r}
$$

Carrying out the algebraic manipulations one obtains

$$
\begin{aligned}
& \mathcal{S}_{M \sigma}=E\left[\begin{array}{ccc}
\mathbf{R}^{T}\left(\phi \phi^{T}+\tilde{\boldsymbol{\kappa}}^{T} \mathbf{I}_{S} \tilde{\boldsymbol{\kappa}}\right) \mathbf{R} & \mathbf{R}^{T} \tilde{\boldsymbol{\kappa}} \mathbf{I}_{S} \tilde{\boldsymbol{\phi}} & \mathbf{R}^{T} \tilde{\boldsymbol{\kappa}} \mathbf{I}_{S} \tilde{\mathcal{K}}_{e} \\
& \tilde{\phi}^{T} \mathbf{I}_{S} \tilde{\boldsymbol{\phi}} & \tilde{\phi}^{T} \mathbf{I}_{S} \tilde{\mathcal{K}}_{e} \\
& & \tilde{\mathcal{K}}_{e}^{T} \mathbf{I}_{S} \tilde{\mathcal{K}}_{e}
\end{array}\right] \\
& \mathcal{S}_{M \tau}=\mu G\left[\begin{array}{ccc}
A \mathbf{R}^{T} \mathbf{I}_{\perp} \mathbf{R} & 0 & A \mathbf{R}^{T} \mathbf{I}_{\perp} \tilde{\boldsymbol{\phi}} \\
& \mathbf{I}_{P} & \mathbf{I}_{P} \tilde{\boldsymbol{\kappa}} \\
\text { symm } & & A \tilde{\phi}^{T} \mathbf{I}_{\perp} \tilde{\phi}+\tilde{\boldsymbol{\kappa}}^{T} \mathbf{I}_{P} \tilde{\boldsymbol{\kappa}}
\end{array}\right], \quad \text { in which } \mathbf{I}_{\perp}=\left[\begin{array}{lll}
0 & 0 & 0 \\
0 & 1 & 0 \\
0 & 0 & 1
\end{array}\right] \text {. }
\end{aligned}
$$

The contribution $\mathbf{R}^{T} \phi \phi^{T} \mathbf{R}$ is the core material stiffness of a TL $3 \mathrm{D}$ bar.

Geometric Stiffness due to Normal Stresses. It is convenient to work out together all geometric stiffness terms produced by the normal stresses, i.e.

$$
\mathcal{S}_{G \sigma}=\mathcal{S}_{G P \sigma}+\mathcal{S}_{G C w \sigma}+\mathcal{S}_{G C q \sigma}=\mathcal{S}_{G w \sigma}+\mathcal{S}_{G C q \sigma}
$$


The appropriate definitions are

$$
\begin{aligned}
& \mathcal{S}_{G P \sigma}=\int_{A_{0}} s_{11} \mathbf{W}_{1}^{T} \mathbf{H} \mathbf{W}_{1} d A, \\
& \mathcal{S}_{G C \sigma}=\int_{A_{0}} s_{11} \mathbf{b}_{1} \delta^{2} \mathbf{g} d A=\delta \mathbf{w}^{T} \mathcal{S}_{G C w \sigma} \delta \mathbf{w}+\mathcal{F}\left(\delta^{2} \mathbf{R}, \delta^{2} \boldsymbol{\kappa}\right),
\end{aligned}
$$

where $\mathcal{F}$ contains $\mathcal{S}_{G C q}$ as $\mathbf{q}$ level kernel. Carrying out the algebraic manipulations one obtains

$$
\mathcal{S}_{G w \sigma}=\mathcal{S}_{G P \sigma}+\mathcal{S}_{G C w \sigma}=\left[\begin{array}{ccc}
\mathcal{P} \mathbf{I} & \mathbf{R}^{T} \widetilde{\mathcal{M}}_{\sigma}^{T} & \mathbf{R}^{T} \tilde{\boldsymbol{\kappa}}^{T} \widetilde{\mathcal{M}}_{\sigma} \\
& 0 & \tilde{\mathcal{M}} \sigma \tilde{\phi} \\
s y m m & & \tilde{\phi} \widetilde{\mathcal{M}}_{\sigma} \tilde{\boldsymbol{\kappa}}+\tilde{\boldsymbol{\kappa}}^{T} \widetilde{\mathcal{M}}_{\sigma} \tilde{\phi}
\end{array}\right]
$$

The term $\mathcal{P I}$ corresponds to the core geometric stiffness of the $3 \mathrm{D} \mathrm{Tl}$ bar.

The higher order term in (10.18) may be expressed as

$$
\mathcal{F}_{\sigma}\left(\delta^{2} \mathbf{R}, \delta^{2} \kappa\right)=\mathcal{M}_{\sigma}^{T} \tilde{\phi} \delta^{2} \kappa+\phi^{T} \mathbf{R} \delta^{2} \mathbf{R}^{T} \tilde{\kappa} \mathcal{M}_{\sigma} \delta \mathbf{q}^{T}\left(\mathbf{V}\left(\tilde{\phi}^{T} \mathcal{M}_{\sigma}\right)+\mathbf{U}\left(\tilde{\boldsymbol{\kappa}} \mathcal{M}_{\sigma} ; \phi\right)\right) \delta \mathbf{q}
$$

Consequently

$$
\mathcal{S}_{G C q \sigma}=\mathbf{V}\left(\tilde{\phi}^{T} \mathcal{M}_{\sigma}\right)+\mathbf{U}\left(\tilde{\boldsymbol{\kappa}} \mathcal{M}_{\sigma} ; \phi\right) .
$$

Because the next-level target variables $q$ include the finite rotation parametrization, matrices $\mathbf{V}$ and $\mathbf{U}$ depend on that choice. They are the source of unsymmetries in the stiffness matrices when certain rotational parametrizations are adopted, such as the incremental rotation vector. If the rotational vector is chosen these matrices are symmetric.

Geometric Stiffness due to Shear Stresses. The contribution of the shear stresses to the geometric stiffness is

$$
\mathcal{S}_{G \tau}=\mathcal{S}_{G P \tau}+\mathcal{S}_{G C w \tau}+\mathcal{S}_{G C q \tau}=\mathcal{S}_{G w \tau}+\mathcal{S}_{G C q \tau} .
$$

The appropriate definitions are

$$
\begin{aligned}
& \mathcal{S}_{G P \tau}=\int_{A_{0}} s_{12}\left(\mathbf{W}_{1}^{T} \mathbf{H} \mathbf{W}_{2}+\mathbf{W}_{2} \mathbf{H} \mathbf{W}_{1}\right)+s_{13}\left(\mathbf{W}_{1}^{T} \mathbf{H} \mathbf{W}_{3}+\mathbf{W}_{3} \mathbf{H} \mathbf{W}_{1}\right) d A \\
& \mathcal{S}_{G C \tau}=\int_{A_{0}}\left(s_{12} \mathbf{b}_{2}+s_{13} \mathbf{b}_{3}\right) \delta^{2} \mathbf{g} d A=\delta \mathbf{w}^{T} \mathcal{S}_{G C w \tau} \delta \mathbf{w}+\mathcal{F}_{\tau}\left(\delta^{2} \mathbf{R}, \delta^{2} \boldsymbol{\kappa}\right) .
\end{aligned}
$$

Carrying out manipulations one obtains the surprisingly simple form for $\mathcal{S}_{G w \tau}$

$$
\mathcal{S}_{G w \tau}=\mathcal{S}_{G P \tau}+\mathcal{S}_{G C w \tau}=\left[\begin{array}{ccc}
0 & 0 & \mathbf{R}^{T} \widetilde{\mathcal{Q}}^{T} \\
& \mathbf{0} & \mathbf{0} \\
s y m m & & \mathbf{0}
\end{array}\right]
$$

The terms due to the second variation of $\mathrm{g}$ become

$$
\mathcal{F}_{\tau}=\mathcal{Q}^{T} \delta^{2} \mathbf{R} \Phi+\mathcal{M}_{\tau}^{T} \delta^{2} \kappa
$$

The kernel carried forward to the $q$ level is

$$
\mathcal{S}_{G C q \tau}=\mathbf{V}\left(\mathcal{M}_{\tau}\right)+\mathbf{U}(\mathcal{Q} ; \boldsymbol{\Phi})
$$




\subsection{Transformation to the Rotational Vector}

The second transformation stage passes from $\mathbf{w}$ to $\mathbf{z}$, which is a vector of generalized displacements, also associated with a beam section, which embodies the parametrization of the cross section rotation:

$$
\mathbf{z}=\left\{\begin{array}{lll}
\frac{d \mathbf{u}_{0}}{d X} & \frac{d \boldsymbol{\alpha}}{d X} & \boldsymbol{\alpha}
\end{array}\right\}^{T}, \quad \delta \mathbf{z}=\left\{\begin{array}{lll}
\frac{d \delta \mathbf{u}_{0}}{d X} & \frac{d \delta \boldsymbol{\alpha}}{d X} & \delta \boldsymbol{\alpha}
\end{array}\right\}^{T} .
$$

Here $\alpha$ denotes the rotational vector parametrization defined by the standard formulas

$$
\boldsymbol{\alpha}=\operatorname{axial}(\tilde{\boldsymbol{\alpha}}), \quad \mathbf{R}=\exp \left(\tilde{\boldsymbol{\alpha}}^{T}\right)
$$

and which may be extracted from $\mathbf{R}$ by

$$
\tilde{\boldsymbol{\alpha}}=\log \mathbf{R}=\frac{\arcsin (\tau)}{2 \tau} \operatorname{axial}\left(\mathbf{R}^{T}-\mathbf{R}\right), \quad \tau=\frac{1}{2}\left\|\operatorname{axial}\left(\mathbf{R}^{T}-\mathbf{R}\right)\right\| .
$$

Because only the variations of $\mathbf{w}$ are known the relation between $\mathbf{w}$ and $\mathbf{z}$ is also of differential type:

$$
\delta \mathbf{w}=\mathbf{Z} \delta \mathbf{z}, \quad \text { or } \quad \delta \mathbf{w}=\left[\begin{array}{ccc}
\mathbf{I} & \mathbf{0} & \mathbf{0} \\
\mathbf{0} & \mathbf{Y}(\mathbf{z}) & \frac{d \mathbf{Y}(\mathbf{z})}{d X} \\
\mathbf{0} & 0 & \mathbf{Y}(\mathbf{z})
\end{array}\right]\left\{\begin{array}{c}
\frac{d \delta \mathbf{u}_{0}}{d X} \\
\frac{d \delta \boldsymbol{\alpha}}{d X} \\
\delta \boldsymbol{\alpha}
\end{array}\right\}
$$

in which

$$
\mathbf{Y}(\alpha)=\frac{\sin |\boldsymbol{\alpha}|}{|\boldsymbol{\alpha}|} \mathbf{I}+\left(1-\frac{\sin |\boldsymbol{\alpha}|}{|\boldsymbol{\alpha}|}\right) \frac{\boldsymbol{\alpha} \boldsymbol{\alpha}^{T}}{|\boldsymbol{\alpha}|^{2}}-\frac{1-\cos |\boldsymbol{\alpha}|}{|\boldsymbol{\alpha}|^{2}} \tilde{\alpha}
$$

On applying the transformations (10.30) we find for the internal force and the material and principal-geometric components of the tangent stiffness matrix:

$$
\mathbf{f}_{q}=\mathbf{Z}^{T}(\mathcal{R} \sigma+\mathcal{R} \tau), \quad \mathbf{K}_{M q}=\mathbf{Z}^{T}\left(\mathcal{S}_{M}\right) \mathbf{Z}, \quad \mathbf{K}_{G P q}=\mathbf{Z}^{T}\left(\mathcal{S}_{G w} \mathbf{Z} .\right.
$$

The materialization of the geometric stiffness terms $\mathcal{S}_{G C q \sigma}$ and $\mathcal{S}_{G C q r}$ for the rotational vector needs additional work. We state here only the final result:

$$
\mathbf{U}(\boldsymbol{\tau} ; \boldsymbol{\Phi})=\left[\begin{array}{ccc}
0 & 0 & 0 \\
& 0 & 0 \\
\text { symm } & & \mathbf{U}^{\tau}
\end{array}\right], \quad \mathbf{T}\left(\mathcal{M}_{\tau}\right)=\left[\begin{array}{ccc}
0 & 0 & 0 \\
& 0 & \mathbf{T}_{1}^{\tau} \\
\text { symm } & & \mathbf{T}_{2}^{\tau}
\end{array}\right]
$$


where

$$
\begin{aligned}
& \mathbf{U}^{\tau}=c_{1} \tau^{T} \mathbf{\Phi} \mathbf{I}+c_{2}\left(\tau \boldsymbol{\Phi}^{T}+\boldsymbol{\Phi}^{T} \boldsymbol{\tau}\right)+c_{3}\left(\tau^{T} \boldsymbol{\Phi} \boldsymbol{\alpha} \boldsymbol{\alpha}^{T}+\tau^{T} \tilde{\boldsymbol{\alpha}} \boldsymbol{\Phi} \mathbf{I}+\tilde{\boldsymbol{\tau}}^{T} \boldsymbol{\Phi} \boldsymbol{\alpha}^{T}+\boldsymbol{\alpha} \boldsymbol{\Phi}^{T} \tilde{\boldsymbol{\tau}}\right) \\
& +c_{5}\left(\boldsymbol{\alpha}^{T} \mathbf{\Phi}\left(\tau \boldsymbol{\alpha}^{T}+\alpha \tau^{T}\right)+\boldsymbol{\alpha}^{T} \tau\left(\boldsymbol{\alpha} \Phi^{T}+\boldsymbol{\Phi} \boldsymbol{\alpha}^{T}\right)+\tau^{T} \boldsymbol{\alpha} \boldsymbol{\alpha}^{T} \mathbf{\Phi} \mathbf{I}\right) \\
& +c_{4} \tau^{T} \tilde{\boldsymbol{\alpha}} \boldsymbol{\Phi} \boldsymbol{\alpha} \boldsymbol{\alpha}^{T}+c_{6} \tau^{T} \boldsymbol{\alpha} \boldsymbol{\alpha}^{T} \boldsymbol{\Phi} \boldsymbol{\alpha} \boldsymbol{\alpha}^{T} \\
& \mathbf{V}_{1}^{\tau}=c_{2} \widetilde{\mathcal{M}}_{\tau}^{T}+c_{3} \alpha \mathcal{M}_{\tau}^{T}+c_{5} \alpha \alpha^{T} \widetilde{\mathcal{M}}_{\tau}+c_{7}\left(\mathcal{M}_{\tau} \alpha^{T}+\alpha^{T} \mathcal{M}_{\tau} I\right)+c_{8} \alpha^{T} \mathcal{M}_{\tau} \alpha \alpha^{T} \text {, } \\
& \mathrm{V}_{2}^{\tau}=-c_{3} \frac{d \alpha^{T}}{d X} \mathcal{M}_{\tau} \mathrm{I}-c_{4} \frac{d \alpha^{T}}{d X} \mathcal{M}_{\tau} \boldsymbol{\alpha} \alpha^{T}+c_{5}\left(\frac{\widetilde{d \alpha}^{T}}{d X} \mathcal{M}_{\tau} \alpha^{T}+\alpha \frac{\widetilde{d \alpha}}{d X} \mathcal{M}_{\tau}^{T}+\alpha^{T} \frac{\widetilde{d \alpha}}{d X} \mathcal{M}_{\tau} \mathbf{I}\right) \\
& +c_{6} \boldsymbol{\alpha}^{T} \frac{\widetilde{d \boldsymbol{\alpha}}}{d X} \mathcal{M}_{\tau} \boldsymbol{\alpha} \boldsymbol{\alpha}^{T}+c_{7}\left(\frac{d \boldsymbol{\alpha}}{d X} \mathcal{M}_{\tau}^{T}+\mathcal{M}_{\tau} \frac{d \boldsymbol{\alpha}^{T}}{d X}\right)+ \\
& +c_{8}{\frac{d \alpha^{T}}{d X}}^{T} \alpha\left(\alpha \mathcal{M}_{\tau}^{T}+\mathcal{M}_{\tau} \alpha^{T}+\alpha^{T} \mathcal{M}_{\tau} \mathbf{I}\right)+c_{9} \frac{d \alpha}{d X}^{T} \alpha \alpha^{T} \mathcal{M}_{\tau} \alpha \alpha^{T},
\end{aligned}
$$

in which

$$
\begin{array}{lll}
c_{1}=-\frac{\sin \alpha}{\alpha}, & c_{2}=\frac{1-\cos \alpha}{\alpha^{2}}, & c_{3}=\frac{\sin \alpha-\alpha \cos \alpha}{\alpha^{3}}, \\
c_{4}=-\frac{c_{1}+3 c_{3}}{\alpha^{2}}, & c_{5}=-\frac{c_{1}+2 c_{2}}{\alpha^{2}}, & c_{6}=-\frac{c_{3}+4 c_{5}}{\alpha^{2}}, \\
c_{7}=\frac{1+c_{1}}{\alpha^{2}}, & c_{8}=\frac{3 c_{3}-2 c_{2}}{\alpha^{2}}, & c_{9}=\frac{c_{5}-5 c_{8}}{\alpha^{2}} .
\end{array}
$$

A similar approach can be taken with (10.30), which defines $\mathcal{F}_{\sigma}$. The tangent stiffness matrix can be obtained by superposing all contributions.

\subsection{Transformation to Finite Element Freedoms}

The final stage introduces a finite element representation for the degrees of freedom. The beam or beam assembly is divided into a set of two-node finite elements. Each of these nodes has three displacement degrees of freedom and three rotational degrees of freedom corresponding to the three $\left\{\alpha_{X}, \alpha_{Y}, \alpha_{Z}\right\}$ components of the rotational vector $\alpha$. Each element in turn has twelve freedoms which are collected in the array $\mathbf{v}^{T}=\left\{\begin{array}{ll}\mathbf{u}_{n} & \alpha_{n}\end{array}\right\}^{T}$ where $\mathbf{d}_{n}$ collects the six translational freedoms while $\alpha_{n}$ collects the six rotations. The cross-section state vector $\mathbf{z}$ is approximated inside each element by

$$
\mathbf{z}=\left[\begin{array}{cc}
\mathbf{N} & \mathbf{0} \\
\mathbf{0} & \frac{d \mathbf{N}}{d X} \\
0 & \mathbf{N}
\end{array}\right]\left\{\begin{array}{l}
\mathbf{d}_{n} \\
\boldsymbol{\alpha}_{n}
\end{array}\right\}=\mathbf{G}_{z}\left\{\begin{array}{l}
\mathbf{d}_{n} \\
\boldsymbol{\alpha}_{n}
\end{array}\right\}=\mathbf{G}_{z} \mathbf{v}
$$

where $\mathbf{N}$ is a matrix of linear shape functions. Since $\delta \mathbf{q}=\mathbf{G}_{z} \delta \mathbf{v}$ the final internal force vector $\mathbf{f}$ and tangent stiffness matrix $\mathbf{K}$ of each element are obtained through the DCCF 
transformations

$$
\mathbf{f}=\int_{0}^{L_{0}} \mathbf{G}_{z}^{T} \mathbf{f}_{z} d X, \quad \mathbf{K}=\int_{0}^{L_{0}} \mathbf{G}_{z}^{T} \mathbf{K}_{z} \mathbf{G}_{z} d X
$$

The choice of shape functions for the rotational vector poses some subtle questions. In small-deflection analysis it is common practice to select all Timoshenko beam shape functions to be linear in $X$. This choice obviously enforces nodal compatibility while preserving constant curvature states. But for finite deflections a linear interpolation for the rotational vector components cannot exactly represent a constant curvature state unless the rotations are about a single axis (plane rotations). The same is true if the rotation matrix $\mathbf{R}(X)$ is interpolated linearly. On the other hand, linear interpolation of Euler parameters does preserve the constant curvature state. This motivated the development of an interpolation scheme that starts from the 4 Euler parameters $\epsilon_{i}(X), i=0,1,2,3$, $\sum_{i} \epsilon_{i}^{2}=1$ that orient the normal of a cross section at $X$. These are collected in the 4 -vector $\epsilon=\left\{\begin{array}{llll}\epsilon_{0} & \epsilon_{1} & \epsilon_{2} & \epsilon_{3}\end{array}\right\}^{T}$. Given the eight end values $\epsilon(0)$ and $\epsilon(L)$ the interpolation that can copy a constant curvature vector $\kappa$ is found to be ${ }^{5}$

$$
\epsilon(\zeta)=\cos (\zeta)\left(1-\frac{\tan (\zeta)}{\tan \left(\zeta_{L}\right)}\right) \epsilon(0)+\frac{\sin (\zeta)}{\sin \left(\zeta_{L}\right)} \epsilon(L),
$$

where $\zeta=\frac{1}{2} \kappa X, \zeta_{L}=\frac{1}{2} \kappa L, \kappa=\sqrt{\kappa^{T} \kappa}$. The constant curvature vector can be extracted from the end values through the formula

$$
\boldsymbol{\kappa}=\frac{1}{\beta_{2} L}\left[\left(\widetilde{\epsilon(L)}-2 \epsilon_{0}(L) \mathbf{I}\right) \epsilon(0)-\left(\widetilde{\epsilon(0)}-2 \epsilon_{0}(0) \mathbf{I}\right) \epsilon(0)\right],
$$

This interpolation is then transformed to the variations in terms of the rotational vector. Details are provided in Reference ${ }^{5}$.

\section{APPLICATION EXAMPLES}

Several application examples solved with the Timoshenko beam elements are described below to show that they do not suffer from the restriction to moderate rotations that several authors attribute the TL description.

\subsection{Cantilevered Beam under End Moment}

This is a classic validation test for geometrically nonlinear beam and shell elements. A cantilevered beam of initial length $L_{0}$ is forced into pure bending by application of an end moment $M$. The beam bends into an arc of circle with curvature $\kappa=M /(E I)$ and end rotation $\theta_{\text {end }}=M L_{0} /(E I)$. The test were run with $E=30.010^{6}, G=E / 2, A=1$, $I=1 / 12, L_{0}=1000$, and $M=15708.4 \lambda$, where $\lambda$ is a load parameter. The $\{X, Y\}$ axes are placed at the left end of the beam. The load scaling is chosen so that for $\lambda=1$ the beam bends to a full circle of radius $L_{0} /(2 \pi)=318.31$. The results for the tip deflection obtained using 10 elements along the length are shown in Table 3 . The results for the $2 \mathrm{D}$ elements ${ }^{10}$ and $3 \mathrm{D}$ elements ${ }^{5}$ were essentially the same within solution acceptance tolerances. The average number of full-Newton iterations per step was 4 . 
Table 3 Computed solutions for plane cantilever beam under pure moment

\begin{tabular}{|crrrrrr|}
\hline Load level & \multicolumn{3}{c}{ Numerical solution } & \multicolumn{3}{c|}{ Analytical solution } \\
\hline$\lambda$ & \multicolumn{1}{c}{$X_{\text {tip }}$} & $Y_{\text {tip }}$ & $\theta_{\text {tip }}$ & $X_{\text {tip }}$ & $Y_{\text {tip }}$ & $\theta_{\text {tip }}$ \\
0.25 & -362.74 & 637.29 & 1.57 & -363.38 & 636.62 & 1.57 \\
0.50 & -1000.03 & 639.23 & 3.14 & -1000.00 & 636.62 & 3.14 \\
0.75 & -1214.18 & 214.15 & 4.71 & -1212.21 & 212.21 & 4.71 \\
1.00 & -999.18 & 0.00 & 4.71 & -1000.00 & 0.00 & 6.28 \\
\hline
\end{tabular}

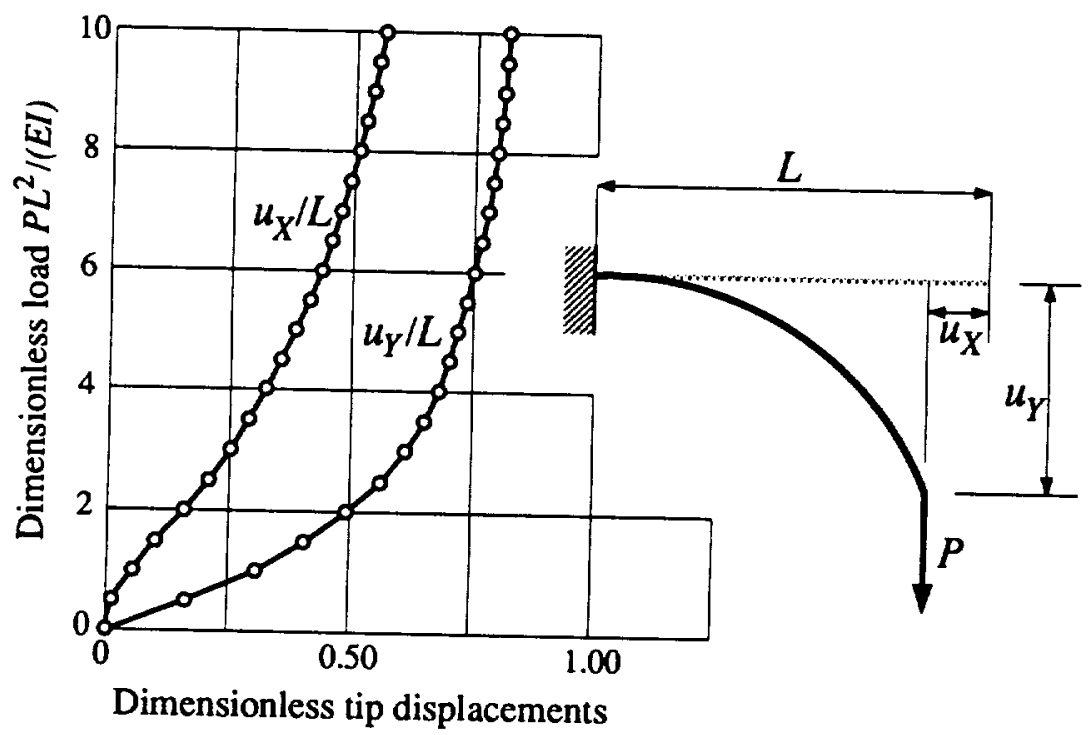

Figure 3. Cantilever under end shear: exact and computed responses

\subsection{Cantilevered Beam Under Shear Load}

A cantilever beam is now subject to a vertical tip load. The results obtained with $162 \mathrm{D}$ Timoshenko TL beam elements in a term project by Abedzadeh, Mehrabi and Lofti ${ }^{11}$ are compared in Figure 3 with the analytical solution given by Mathiasson et.al. ${ }^{8}$ The $2 \mathrm{D}$ Timoshenko elements follow the exact solution without appreciable error.

REMARK 11.1. As noted in Remark 1.1, Mathiasson et. al. ${ }^{8}$ reported fair to poor results beyond moderate rotations in this problem using a TL-based 2D Hermitian beam element. The difficulty can be traced to their use of an approximate expression for the curvature:

$$
\kappa \approx-\frac{u_{Y}^{\prime \prime}}{\left[1+\left(u_{Y}^{\prime}\right)^{2}\right]^{3 / 2}}
$$

where primes denote derivatives with respect to $X$, instead of the correct small-strain TL value

$$
\kappa=\frac{d \theta}{d X}=\theta^{\prime}=-\frac{u_{Y}^{\prime \prime}\left(1+u_{X}^{\prime}\right)-u_{X}^{\prime \prime} u_{Y}^{\prime}}{\left[1+\left(u_{X}^{\prime}\right)^{2}+\left(u_{Y}^{\prime}\right)^{2}\right]^{3 / 2}} .
$$



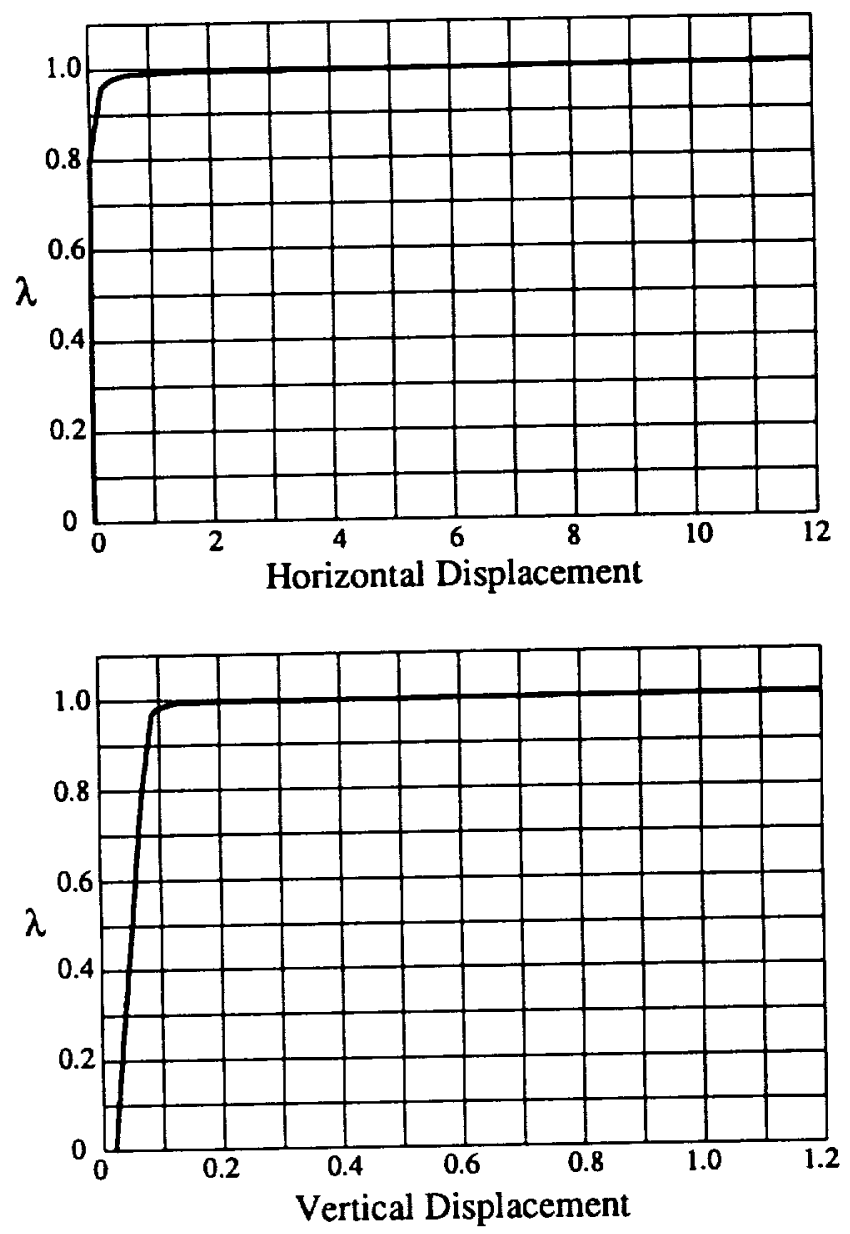

Figure 4. Buckling of cantilever column: load-displacement responses

The expression (11.2) rapidly losses accuracy as $u_{X}^{\prime}$ and $u_{X}^{\prime \prime}$ increase. Since (11.1) usually overestimates the actual curvature, it tends to overstiffen the element.

\subsection{Euler Buckling of Cantilever Column}

This buckling example is taken from Mathisen's thesis. ${ }^{12}$ The critical buckling load was traversed by treating the bifurcation point as a limit point by introducing a slight geometric imperfection. The following inputs were used in the study reported in Alexander et.al. ${ }^{10}$ : $L_{0}=100, A=0.05, I=0.20, E=10^{6}, G=E / 2$ and applied load $P=49.34 \lambda$. The load response curves using the axial displacement as control variable are shown in Figure 4. The computed results agree well with elastica solutions up to deflections of the order of the column length.

\subsection{Large Displacement of a $45^{\circ}$ Cantilever Bend}

This 3D beam problem concerns the large displacement analysis of a $45^{\circ}$ cantilever bend initially lying on the horizontal $\{X, Y\}$ plane. The bend is an arc of a circle of radius $r=100$ and the beam cross-section is a square with sides of unit length. The beam has modulus $E=10^{7}$ and Poisson's ratio $\nu=0$. It is subjected to an end load $P=600$ normal 


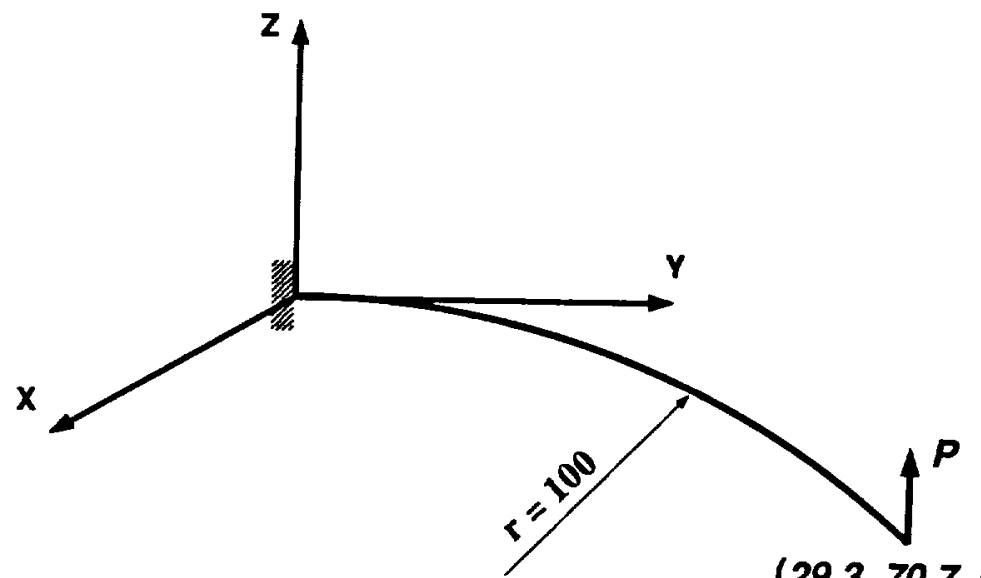

$(29.3,70.7,0.0)$

Figure 5. Curved cantilever bend under tip load: Problem definition

to the $(X, Y)$ plane as shown in Figure 5.

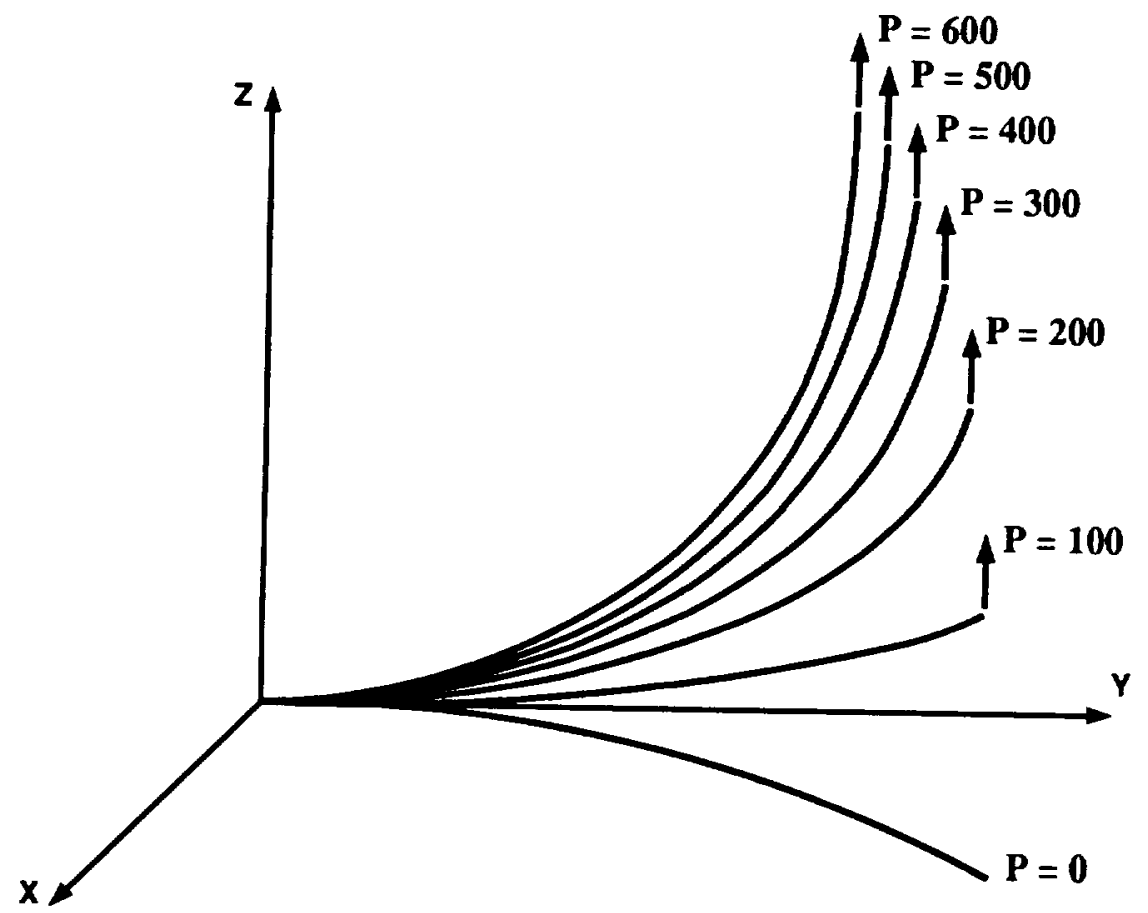

Figure 6. Curved cantilever bend under tip load: Deflected shapes

This problem was treated by Bathe and Bolourchi ${ }^{13}$ with $3 \mathrm{D}$ brick elements, and subsequently by Simo and Vu-Quoc ${ }^{14}$ and Cardona ${ }^{15}$ with beam elements based on other formulations. Results with the TL 3D element described in Sections 5.5 and 10 were ob- 
Table 4. Comparison of results for the $45^{\circ}$ bend cantilever beam

\begin{tabular}{|lrrr|}
\hline Source & Load $P=300$ & Load $P=450$ & Load $P=600$ \\
\hline Bathe $^{13}$ & $22.33,58.84,40.08$ & $18.62,53.32,48.39$ & $15.79,47.23,53.37$ \\
Simo $^{14}$ & $22.50,59.20,39.50$ & & $15.90,47.20,53.40$ \\
Cardona $^{15}$ & $22.14,58.64,40.35$ & $18.38,52.11,48.59$ & $15.55,47.04,53.50$ \\
Crivelli $^{5,7}$ & $22.31,58.85,40.08$ & $18.59,53.34,48.39$ & $15.75,47.25,53.37$ \\
\hline
\end{tabular}

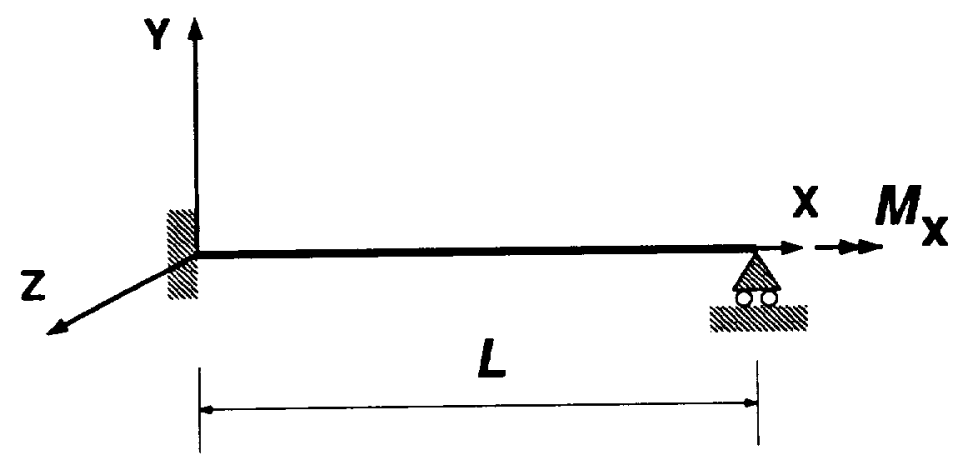

$\begin{array}{ll}\text { Length } & L=240 \mathrm{~mm} \\ \text { Polar Moment } & I_{x}=2.16 \mathrm{~mm}^{4} \\ \text { Second Moments } & I_{y}=I_{z}=0.0833 \mathrm{~mm}^{4} \\ \text { Young's Modulus } & E=71240 \mathrm{~N} / \mathrm{mm}^{2} \\ \text { Shear Modulus } & G=27190 \mathrm{~N} / \mathrm{mm}^{2}\end{array}$

Figure 7. Cable hockling: Problem definition

tained by Crivelli $^{5}$ using 8 beam elements and applying the load in 6 equal increments. The solution method is incremental-iterative with full Newton iteration used in the corrective phase. Results for the three tip displacement components are compared with those of the aforementioned references in Table 4. Deflected shapes for selected load levels are shown in Figure 6.

As can be observed the present results compare especially well with those obtained with $3 \mathrm{D}$ elements in Bathe and Bolourchi ${ }^{13}$.

\subsection{Cable Hockling}

The second problem, cable hockling, is more challenging as regards modeling and postbuckling response analysis. An initially straight cable, modeled with 3D Timoshenko beam elements, is subjected to a tip torsional moment. The geometry and physical properties are given in Figure 7 . The cable is clamped at one end and supported at the other so 
that the only motions allowed at that point are axial displacement and torsional rotation about the longitudinal $X$ axis. No rotation is allowed about $Y$ or $Z$. The purpose of these restrictions is to keep the problem conservative, because if the torque is allowed to rotate about $Y$ or $Z$, the problem becomes nonconservative and dynamical methods are required to assess its stability. ${ }^{16,17}$

This problem has received a great deal of attention from the engineering community due to its practical importance. The main objective is to estimate the critical applied torque at which the cable departs (bifurcates) from its straight configuration, resulting in the formation of a loop or hockle. This has direct application to marine cables used in tasks such as lifting objects from the ocean floor, for which structural failure could be disastrous. Under the assumptions of infinitesimal bending deformations, Greenhill obtained an analytical

formula to predict the critical torque; see e.g. pp. $417-418$ of Love. ${ }^{18}$ The post-bifurcation response analysis of this problem, however, has not been pursued until recently, as discussed in the research conducted by Nour-Omid and Rankin. ${ }^{19}$ This post-critical response has also been analyzed by using the present TL formulation. The structure is discretized by twenty equally-spaced beam elements.

The deformed shapes at different load levels are shown in Figure 8, which displays the loop-formation process previously described. The curves on the left and right side show deformed shapes looking along the $Y$ and $Z$ axes, respectively.

If the torque is held under the critical value, the beam twists without lateral deflection. Along this fundamental path the response is linear. At the critical torque a bifurcation point is reached, at which the fundamental path becomes unstable. The beam acquires a helical shape with the free end moving towards the clamped end. This new equilibrium branch is unstable and the cable undergoes large displacements and rotations as the moment decreases. The unloading process continues until reaching a sharp limit point which corresponds to a negative value of the applied torque (it should be mentioned that Nour-Omid and Rankin, ${ }^{19}$ who use a Hermitian beam element based on a corotational description, characterize this critical point as a secondary bifurcation; evidence for this classification is presently inconclusive). After traversing this point, the torque reverses again until the cable reaches a circular-shaped unloaded deformed configuration.

The computed variation of the twist angle at the moving end versus the applied torque is given in Figure 9. Results are compared to those given by Nour-Omid and Rankin.

\section{CONCLUDING REMARKS}

This article covers an alternative technique for formulating geometrically nonlinear mechanical finite elements based on the Total Lagrangian kinematic description. The CoreCongruential Formulation, or CCF, can be approached and studied at several levels of complexity. These give rise to what are called here the Direct, Algebraic-Generalized, and Differential-Generalized CCF.

All of these variants, however, share a basic staged approach to the derivation of discrete 


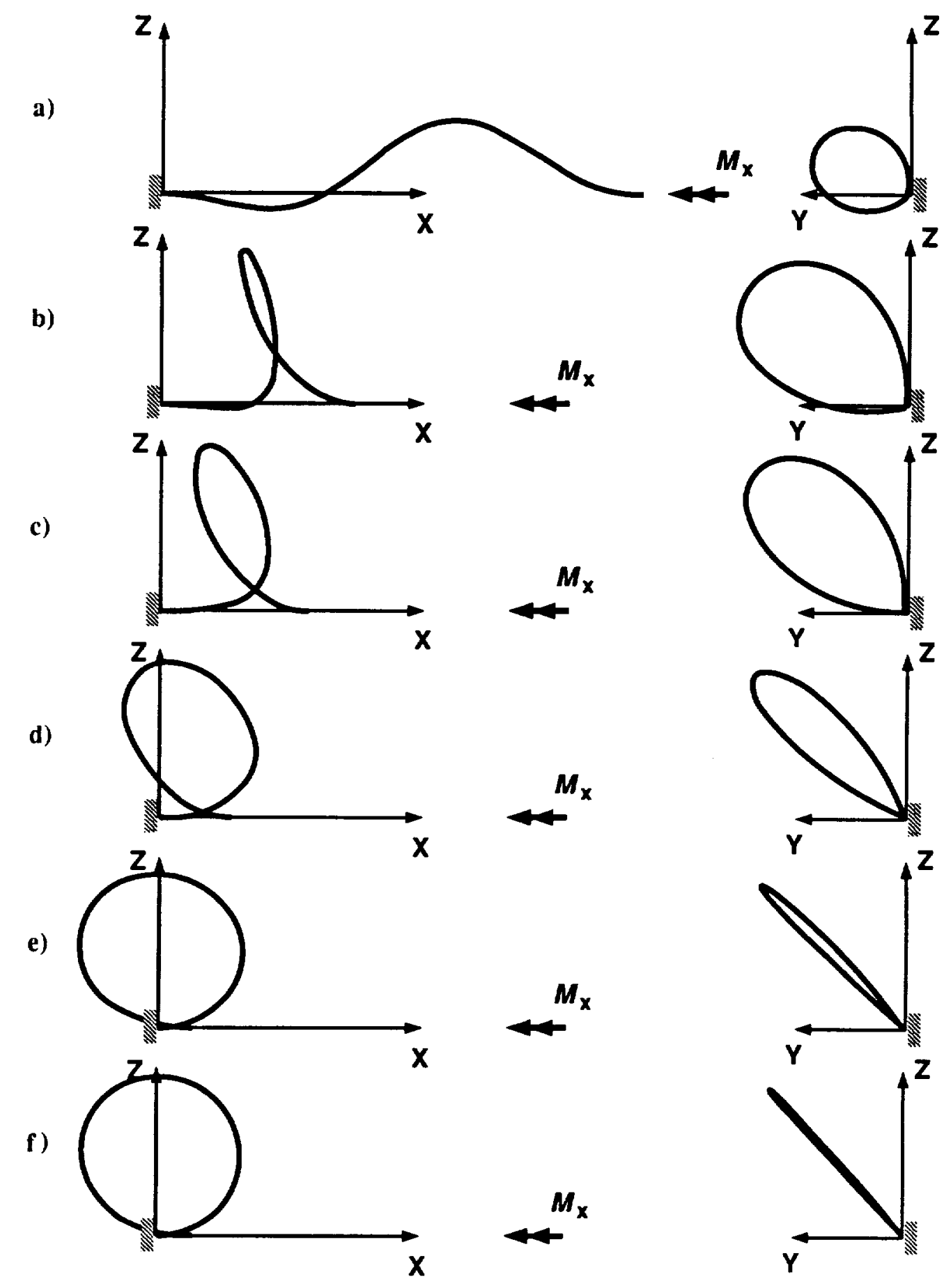

Figure 8. Cable hockling: Deformed shapes at different load levels:

a) After bifurcation, b) $M_{x}=50$, c) $M_{x}=0$, d) $M_{x}=-50$,

e) At the limit point, f) $M_{x}=0$.

finite element equations. In the innermost level, core equations are obtained at the particle level. These physically transparent equations depend only on the strain and stress measures adopted and the choice of terms retained in the strain energy. This is followed by a 


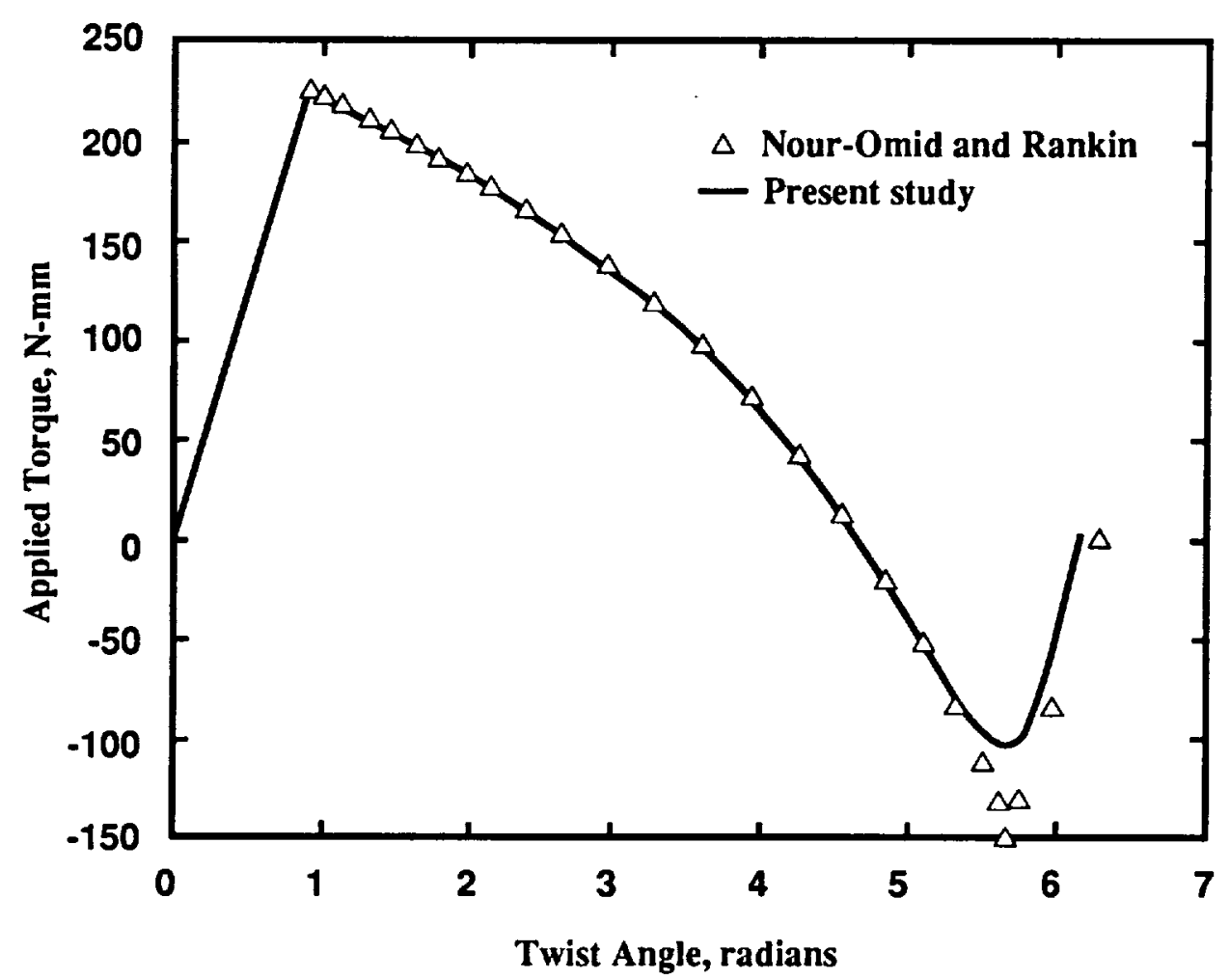

Figure 9. Cable hockling: Computed response as tip twist rotation vs. applied moment

transformation phase that ultimately ends in the element nodal degrees of freedom. For complex elements the transformation phase is frequently carried out in stages.

For elements whose nodal degrees of freedom include 3D finite rotations, a multistage transformation is convenient in the sense that the decision on which finite rotation measure to use can be relegated to the next-to-last stage, while the choice of finite element interpolation and nodal degrees of freedom is introduced in the last stage. This strategy facilitate application of inner-level equations to sets of different but related elements and fosters programming modularity.

At this point one may well pose general questions such as: Why use the Total Lagrangian description? Wouldn't a co-rotational or Updated Lagrangian formulation be preferable? The answer is that each description has strengths and weaknesses. Here are advantages that can be cited for the TL description:

1. If the element development can be carried out under the framework of the DCCF or AGCCF, a symmetric tangent stiffness formulation is guaranteed. This attribute has obvious advantages in stability analysis and traversal of bifurcation points.

2. The choice of a fixed reference frame has advantages in nonlinear dynamic calculations in that the mass matrix remains symmetric, with the same sparsity as in small-deflection analysis. 
3. The use of Green-Lagrange strains and conjugate PK2 stresses, linked up with careful avoidance of hazardous kinematic approximations, automatically takes care of rigid body motions. No special filters to eliminate self-straining at the element level are needed.

4. Extension to nonlinear constitutive equations and finite strains is straightforward although may be laborious. In the case of the CCF, extension to small-strain material nonlinearity would affect only the core equations because the transformation phase is entirely governed by element kinematics.

The TL description, however, suffers from several shortcomings:

5. It makes no effective reuse of existing linear finite elements. This is the key strength of the element-independent corotational description. ${ }^{19,20}$

6. Has difficulties with the specification and computational treatment of boundary conditions intrinsically linked to the deformed configuration; for example pressure loads. Both the corotational and Updated Lagrangian descriptions handle this aspect better.

7. Eventually breaks down for exceedingly large motions, for example rotations exceeding $2 \pi$. This disadvantage can be important for certain aerospace and mechanical structures.

From this list it follows that there is no "kinematic description for all seasons." One intriguing research area that may merit exploration in this regard is a combination of the TL and corotational descriptions that maintains their individual strengths while alleviating the more serious disadvantages.

\section{Acknowledgements}

The research work surveyed in this article has been supported by the Air Force Office of Scientific Research under Grant F49620-87-C-0074, NASA Langley Research Center under Grant NAG1756, NASA Lewis Research Center under Grant NAG3-1273, and the National Science Foundation under Grant ASC-9217394.

\section{References}

1. S. Rajasekaran and D. W. Murray, Incremental finite element matrices, J. Str. Div. ASCE, 99, pp. 2423-2438, 1973.

2. R. H. Mallet and P. V. Marcal, Finite element analysis of nonlinear structures, $J$. Str. Div. ASCE, 94, pp. 2081-2105, 1968.

3. D. W. Murray, Finite element nonlinear analysis of plates, Ph. D. Dissertation, Dept. of Civil Engineering, University of California, Berkeley, California, 1967.

4. C. A. Felippa, Discussion of Reference 1, J. Str. Div. ASCE, 100, pp. 2519-2521, 1974.

5. L. A. Crivelli, A Total-Lagrangian beam element for analysis of nonlinear space structures, Ph. D. Dissertation, Dept. of Aerospace Engineering Sciences, University of Colorado, Boulder, CO, 1990. 
6. C. A. Felippa and L. A. Crivelli, A congruential formulation of nonlinear finite elements, in Nonlinear Computational Mechanics - The State of the Art, ed. by P. Wriggers and W. Wagner, Springer-Verlag, Berlin, pp. 283-302, 1991.

7. L. A. Crivelli and C. A. Felippa, A three-dimensional non-linear Timoshenko beam element based on the core-congruential formulation, Int. J. Numer. Meth. Engrg., 36, pp. 3647-3673,
1993.

8. K. Mathiasson, A. Bengtsson and A. Samuelsson, On the accuracy and efficiency of numerical algorithms for geometrically nonlinear structural analysis, in Finite Element Methods for Nonlinear Problems, ed. by P. G. Bergan, K. J. Bathe and W. Wunderlich, Springer-Verlag,
Berlin, pp. 3-24, 1986.

9. P. G. Bergan and K. M. Mathisen, Large displacement analysis of highly flexible offshore structures, in Nonlinear Computational Mechanics - The State of the Art, ed. by P. Wriggers and W. Wagner, Springer-Verlag, Berlin, pp. 303-331.

10. S. Alexander, H. M. de la Fuente and B. Haugen, Correspondence between CC-TL and C-TL formulations A 2D Timoshenko beam element using the Total-Lagrangian Core Congruential Formulation, in Term Projects in Nonlinear Finite Element Methods, ed. by C. A. Felippa, Report CU-CSSC-91-12, Center for Space Structures and Controls, University of Colorado, Boulder, CO, May 1991.

11. F. Abedzadeh Anaraki, A. Barzegar Mehrabi and H. R. Lofti, Correspondence between CCTL and C-TL formulations, in Term Projects in Nonlinear Finite Element Methods, ed. by C. A. Felippa, Report CU-CSSC-91-12, Center for Space Structures and Controls, University of Colorado, Boulder, CO, May 1991.

12. K. M. Mathisen, Large displacement analysis of flexible and rigid systems considering displacement-dependent loads and nonlinear constraints, Dr. Ing. Thesis, Div. of Structural Mechanics, The Norwegian Institute of Technology, Trondheim, Norway, 1990

13. K. J. Bathe and S. Bolourchi, Large displacement analysis of three-dimensional beam structures, Int. J. Numer. Meth. Engrg., 14, pp. 961-986, 1979.

14. J. C. Simo and L. Vu-Quoc, A three-dimensional finite strain rod model. Part II: Computational aspects, Comp. Meths. Appl. Mech. Engrg., 58, pp. 79-116, 1986.

15. A. Cardona, An integrated approach to mechanism analysis, Ph. D. Dissertation, LTAS, Université de Liège, Belgium, 1989.

16. V. V. Bolotin, Nonconservative Problems of the Theory of Elastic Stability, Pergamon Press,
New York, 1963.

17. H. Ziegler, Principles of Structural Stability, Blaisdell, Massachusetts, 1968.

18. A. E. H. Love, The Mathematical Theory of Elasticity, Dover, New York, 1944.

19. B. Nour-Omid and C. C. Rankin, Finite rotation analysis and consistent linearization using projectors, Comp. Meths. Appl. Mech. Engrg., 93, pp. 353-384, 1991.

20. B. Haugen, Buckling and stability problems for thin shell structures using high-performance finite elements, Ph. D. Dissertation, Dept. of Aerospace Engineering Sciences, University of
Colorado, Boulder, CO, 1994 .

21. O. C. Zienkiewicz, The Finite Element Method, $3^{\text {rd }}$ ed., McGraw-Hill, London, 1976. 


\section{Appendix 1}

\section{Equivalence of DCCF and Standard TL Formulation}

The correspondence between the Direct Core Congruential Formulation (DCCF) and the Standard Formulation (SF) of the Total Lagrangian (TL) kinematic description is generally established for 3D continuum finite elements. This connection was worked out in a course term project. ${ }^{11}$ Such elements fit within the DCCF framework because their physical DOFs (node displacements) are of translational type.

The Standard Formulation is based on the same scheme used for linear finite elements: first interpolate, then vary. As in the linear case, the departure point is extremization of the Total Potential Energy functional (TPE) over the element domain:

$$
J=U-W=\int_{V_{0}} \mathbf{e}^{T} \mathbf{s}_{0} d V+\frac{1}{2} \int_{V_{0}} \mathbf{e}^{T} \mathbf{E} \mathbf{e} d V-\int_{V_{0}} \mathbf{u}^{T} \mathbf{b} d V-\int_{S_{\mathbf{t} 0}} \mathbf{u}^{T} \mathbf{t} d S
$$

where as usual conservative dead loading is assumed. In (A1.1), b is the prescribed body force field, $t$ are surface tractions prescribed over portion $S_{10}$ of the boundary in $\mathcal{C}_{0}$, and other quantities are as defined in Section 4. The weak equilibrium equations are obtained on making (A1.1) stationary:

$$
\delta J=\delta U-\delta W=\int_{V_{0}} \delta \mathbf{e}^{T} \mathbf{s}_{0} d V+\int_{V_{0}} \delta \mathbf{e}^{T} \mathbf{E} \mathbf{e} d V-\int_{V_{0}} \delta \mathbf{u}^{T} \mathbf{b} d V-\int_{S_{t_{0}}} \delta \mathbf{u}^{T} \mathbf{t} d S=\mathbf{0} .
$$

The displacement and strain fields are interpolated in terms of the element degrees of freedom $\mathbf{v}$ :

$$
\mathbf{u}=\mathbf{N} \mathbf{v}, \quad \delta \mathbf{u}=\mathbf{N} \delta \mathbf{v}, \quad \delta \mathbf{e}=\mathbf{B} \delta \mathbf{v},
$$

where $\mathbf{B}=\mathbf{B}(\mathbf{v})$ depends in $\mathbf{v}$ but $\mathbf{N}$ does not. Substituting these interpolations into (A1.2) yields the residual equilibrium equations

$$
\delta J=\delta \mathbf{v}^{T} \mathbf{r}=\delta \mathbf{v}^{T}(\mathbf{f}-\mathbf{p})=\mathbf{0} .
$$

where

$$
\mathbf{f}=\int_{V_{0}} \mathbf{B}^{T}\left(\mathbf{s}_{0}+\mathbf{E} \mathbf{e}\right) d V=\int_{V_{0}} \mathbf{B}^{T} \mathbf{s} d V, \quad \mathbf{p}=\int_{V_{0}} \mathbf{N}^{T} \mathbf{b} d V+\int_{s_{\mathrm{t} 0}} \mathbf{N} \mathbf{s} d S,
$$

where $f$ and $p$ are the internal and external force vectors, respectively, and $s=s^{0}+$ Ee are the $\mathrm{PK} 2$ stresses in $\mathcal{C}$. Because the variations $\delta \mathrm{v}$ are arbitrary, the residual-force nonlinear equilibrium equation is $\mathbf{r}=\mathbf{f}-\mathbf{p}=\mathbf{0}$ or $\mathbf{f}=\mathbf{p}$. The tangent stiffness matrix is given by

$$
\mathbf{K}=\frac{\partial \mathbf{r}}{\partial \mathbf{v}}=\frac{\partial \mathbf{f}}{\partial \mathbf{v}}
$$

because $\mathbf{p}$ (for conservative dead loading) does not depend on $\mathbf{v}$. Splitting $\mathbf{B}=\mathbf{B}_{c}+\mathbf{B}_{v}(\mathbf{v}$ ), where $\mathbf{B}_{c}$ is constant but $\mathbf{B}_{v}$ depends on $\mathbf{v}$, gives the well known decomposition

$$
\mathbf{K}=\mathbf{K}_{0}+\mathbf{K}_{D}+\mathbf{K}_{G}
$$


where $\mathbf{K}_{0}, \mathbf{K}_{D}$ and $\mathbf{K}_{G}$ denote the linear, initial-displacement and geometric stiffness matrices, respectively. These are given by

$$
\begin{aligned}
\mathbf{K}_{0} & =\int_{V_{0}} \mathbf{B}_{c}^{T} \mathbf{E B}_{c} d V \\
\mathbf{K}_{D} & =\int_{V_{0}}\left(\mathbf{B}_{c}^{T} \mathbf{E B}_{v}+\mathbf{B}_{v}^{T} \mathbf{E B}_{c}+\mathbf{B}_{v}^{T} \mathbf{E B}_{v}\right) d V \\
\mathbf{K}_{G} \delta \mathbf{v} & =\int_{V_{0}} \delta \mathbf{B}^{T} \mathbf{s} d V .
\end{aligned}
$$

To correlate these standard forms with those produced by the DCCF, we note that the GL strains can be also split as $\mathbf{e}=\mathbf{e}_{c}+\mathbf{e}_{v}$, where $\mathbf{e}_{c}$ and $\mathbf{e}_{v}$ are linear and nonlinear in $\mathbf{v}$, respectively. The latter may be expressed in terms of the displacement gradients as

$$
\mathbf{e}_{v}=\frac{1}{2} \mathbf{A g}
$$

where $A$ is the $6 \times 9$ matrix

$$
\mathbf{A}=\left[\begin{array}{ccc}
\mathbf{g}_{1}^{T} & \mathbf{0} & \mathbf{0} \\
\mathbf{0} & \mathbf{g}_{2}^{T} & 0 \\
\mathbf{0} & \mathbf{0} & \mathbf{g}_{3}^{T} \\
\mathbf{0} & \mathbf{g}_{3}^{T} & \mathbf{g}_{2}^{T} \\
\mathbf{g}_{3}^{T} & \mathbf{0} & \mathbf{g}_{1}^{T} \\
\mathbf{g}_{2}^{T} & \mathbf{g}_{1}^{T} & \mathbf{0}
\end{array}\right]=\left[\begin{array}{ccccccccc}
g_{1} & g_{2} & g_{3} & 0 & 0 & 0 & 0 & 0 & 0 \\
0 & 0 & 0 & g_{4} & g_{5} & g_{6} & 0 & \mathbf{0} & 0 \\
0 & 0 & 0 & 0 & 0 & 0 & g_{7} & g_{8} & g_{9} \\
0 & 0 & 0 & g_{7} & g_{8} & g_{9} & g_{4} & g_{5} & g_{6} \\
g_{7} & g_{8} & g_{9} & 0 & 0 & 0 & g_{1} & g_{2} & g_{3} \\
g_{4} & g_{5} & g_{6} & g_{1} & g_{2} & g_{3} & 0 & 0 & 0
\end{array}\right]
$$

in which the displacement gradients are vector-arranged as

$$
\mathbf{g}^{T}=\left[\begin{array}{lllll}
g_{1} & g_{2} & \cdots & g_{8} & g_{9}
\end{array}\right]=\left[\begin{array}{lllll}
\frac{\partial u_{1}}{\partial X_{1}} & \frac{\partial u_{2}}{\partial X_{1}} & \cdots & \frac{\partial u_{2}}{\partial X_{3}} & \frac{\partial u_{3}}{\partial X_{3}}
\end{array}\right]
$$

Comparing

$$
\delta \mathbf{e}_{v}=\frac{1}{2} \delta \mathbf{A} \mathbf{g}+\frac{1}{2} \mathbf{A} \delta \mathbf{g}=\mathbf{A} \delta \mathbf{g}
$$

to the DCCF transformation relation $\delta \mathrm{g}=\mathrm{G} \delta \mathbf{v}$, in which $\mathbf{G}$ is independent of $\mathbf{v}$, we see that

$$
\mathbf{B}_{v}=\mathbf{A} \mathbf{G} \text {. }
$$

The other expression we require is $\delta \mathbf{A}^{T} \mathbf{s}$, which appears in the geometric stiffness matrix contracted with $\delta \mathrm{v}$ :

$$
\mathbf{K}_{G} \delta \mathbf{v}=\int_{V_{0}} \delta \mathbf{B}^{T} \mathbf{s} d V=\int_{V_{0}} \mathbf{G}^{T} \delta \mathbf{A}^{T} \mathrm{~s} d V .
$$

It is well known - see for instance Chapter 19 of Zienkiewicz ${ }^{21}$ - that

$$
\delta \mathbf{A}^{T} \mathbf{s}=\mathbf{M} \delta \mathbf{g}=\mathbf{M G} \delta \mathbf{v}, \quad \text { with } \quad \mathbf{M}=\left[\begin{array}{lll}
s_{1} I & s_{4} I & s_{5} I \\
s_{4} I & s_{2} I & s_{6} I \\
s_{5} I & s_{6} I & s_{3} I
\end{array}\right]
$$


where $I$ is the $3 \times 3$ identity matrix and $s_{i}, i=1, \ldots 6$ are components of the PK2 stress tensor ordered $s_{1}=s_{11}, s_{2}=s_{22}, \ldots s_{6}=s_{23}$. Using this relation, $\mathbf{K}_{G}$ can be placed in the standard form

$$
\mathbf{K}_{G}=\int_{V_{0}} \mathbf{G}^{T} \mathbf{M G} d V,
$$

which by inspection is seen to be the DCCF-transformation of the core geometric stiffness $\mathbf{M} \equiv$ $\mathbf{S}_{G}=s_{i} \mathbf{H}_{i}$, with the $\mathbf{H}_{i}$ matrices defined in (4.3).

To correlate other terms, write the linear part of the GL strains in terms of gradients as

$$
\mathbf{e}_{c}=\mathbf{D g}=\mathbf{D G} \delta \mathbf{v}, \quad \text { with } \quad \mathbf{D}=\left[\begin{array}{lllllllll}
1 & 0 & 0 & 0 & 0 & 0 & 0 & 0 & 0 \\
0 & 0 & 0 & 0 & 1 & 0 & 0 & 0 & 0 \\
0 & 0 & 0 & 0 & 0 & 0 & 0 & 0 & 1 \\
0 & 1 & 0 & 1 & 0 & 0 & 0 & 0 & 0 \\
0 & 0 & 1 & 0 & 0 & 0 & 1 & 0 & 0 \\
0 & 0 & 0 & 0 & 0 & 1 & 0 & 1 & 0
\end{array}\right]
$$

The numerical $D$ matrix can be easily related to the $h_{i}$ vectors introduced in (4.3). Because both $\mathbf{D}$ and $\mathbf{G}$ are independent of $\mathbf{v}$ it follows that $\delta \mathbf{e}_{c}=\mathbf{D G} \delta \mathbf{v}$ and consequently $\mathbf{B}_{c}=\mathbf{D G}$. Partitioning $\mathbf{A}$ as $\left[\begin{array}{llll}\mathbf{a}_{1}^{T} & \mathbf{a}_{2}^{T} & \ldots & \mathbf{a}_{6}^{T}\end{array}\right]$ one easily finds that $\mathbf{a}_{\mathbf{i}}=\mathbf{H}_{\mathbf{i}} \mathbf{g}$. Now the following identities can be verified through simple algebra:

$$
\begin{aligned}
\mathbf{D}^{T} \mathbf{E D} & =E_{i j} \mathbf{h}_{i} \mathbf{h}_{i}^{T}=\mathbf{S}_{0}, \\
\mathbf{D}^{T} \mathbf{E A} & =E_{i j} \mathbf{h}_{i} \mathbf{a}_{j}^{T}=E_{i j} \mathbf{h}_{i} \mathbf{g}^{T} \mathbf{H}_{j}=\mathbf{S}_{1}, \quad \mathbf{A}^{T} \mathbf{E D}=\mathbf{S}_{1}^{T}, \\
\mathbf{A}^{T} \mathbf{E A} & =E_{i j} \mathbf{a}_{i} \mathbf{a}_{j}=E_{i j} \mathbf{H}_{i} \mathbf{g}_{i} \mathbf{g}_{j}^{T} \mathbf{H}_{j}=\mathbf{S}_{2}=\mathbf{S}_{2}^{T}, \\
\mathbf{M} & =s_{i}^{0} \mathbf{H}_{i}+E_{i j} \mathbf{h}_{i} \mathbf{g} \mathbf{H}_{j}+\frac{1}{2}\left(\mathbf{g}^{T} \mathbf{H}_{i} \mathbf{g}\right) \mathbf{H}_{j}=s_{i}^{0} \mathbf{H}_{i}+\mathbf{S}_{i}^{*}+\frac{1}{2} \mathbf{S}_{2}^{*}=s_{i} \mathbf{H}_{i} .
\end{aligned}
$$

Comparing these to the expressions of Section 4.3 we conclude that

$$
\begin{aligned}
\mathbf{K}_{0} & =\int_{V_{0}} \mathbf{G}^{T} \mathbf{D}^{T} \mathbf{E D G} d V=\int_{V_{0}} \mathbf{G}^{T} \mathbf{S}_{0} \mathbf{G} d V, \\
\mathbf{K}_{D} & =\int_{V_{0}} \mathbf{G}^{T}\left(\mathbf{D}^{T} \mathbf{E A}+\mathbf{A}^{T} \mathbf{E D}+\mathbf{A}^{T} \mathbf{E A}\right) \mathbf{G} d V=\int_{V_{0}} \mathbf{G}^{T} \mathbf{S}_{D} \mathbf{G} d V, \\
\mathbf{K}_{G} & =\int_{V_{0}} \mathbf{G}^{T} \mathbf{M} \mathbf{G} d V=\int_{V_{0}} \mathbf{G}^{T} \mathbf{S}_{G} \mathbf{G} d V,
\end{aligned}
$$

which displays the equivalence of both formulations when no approximations are made. This proof may be extended without difficulty to the AGCCF in which case $G$ is a function of $v$, although as noted in the text that situation is sometimes mishandled in the Standard Formulation through the introduction of a priori kinematic approximations. The equivalence between DGCCF and SF is more difficult to prove because there is no TPE functional from which the latter can be derived, and such connection should be regarded as an open problem. 


\section{Appendix 2}

\section{Tangent Stiffness Subroutine for Two-Node 3D Bar}

The subroutine listed below implements the expression derived in Section 7.1 for the global tangent stiffness of a two-noded TL 3D bar element. The Fortran implementation is biased in favor of computational speed. Therefore, it contain no loops or calls to other subroutines, as that would slow down the calculations. The formation of the $6 \times 6$ tangent stiffness matrix requires 74 multiplications, 14 divisions, 64 additions/subtractions and 3 square roots, for a total of approximately 160 double precision floating-point operations. On a 15-MFlop workstation, this results in approximately 50000 elements formed per CPU second.

One point that deserves some attention is the numerically-stable choice of the local coordinate system. Axis $\bar{X}$ is uniquely defined as the longitudinal bar axis that passes through the end nodes, but axes $\bar{Y}$ and $\bar{Z}$ may be arbitrarily rotated about $\bar{X}$ because a 3D bar has no preferred transverse directions. Although the end result, namely $\mathbf{K}$, is independent of this choice it is important to choose $\bar{Y}$ and $\bar{Z}$ in a stable manner that works for any element orientation. The procedure is described in code comments below.

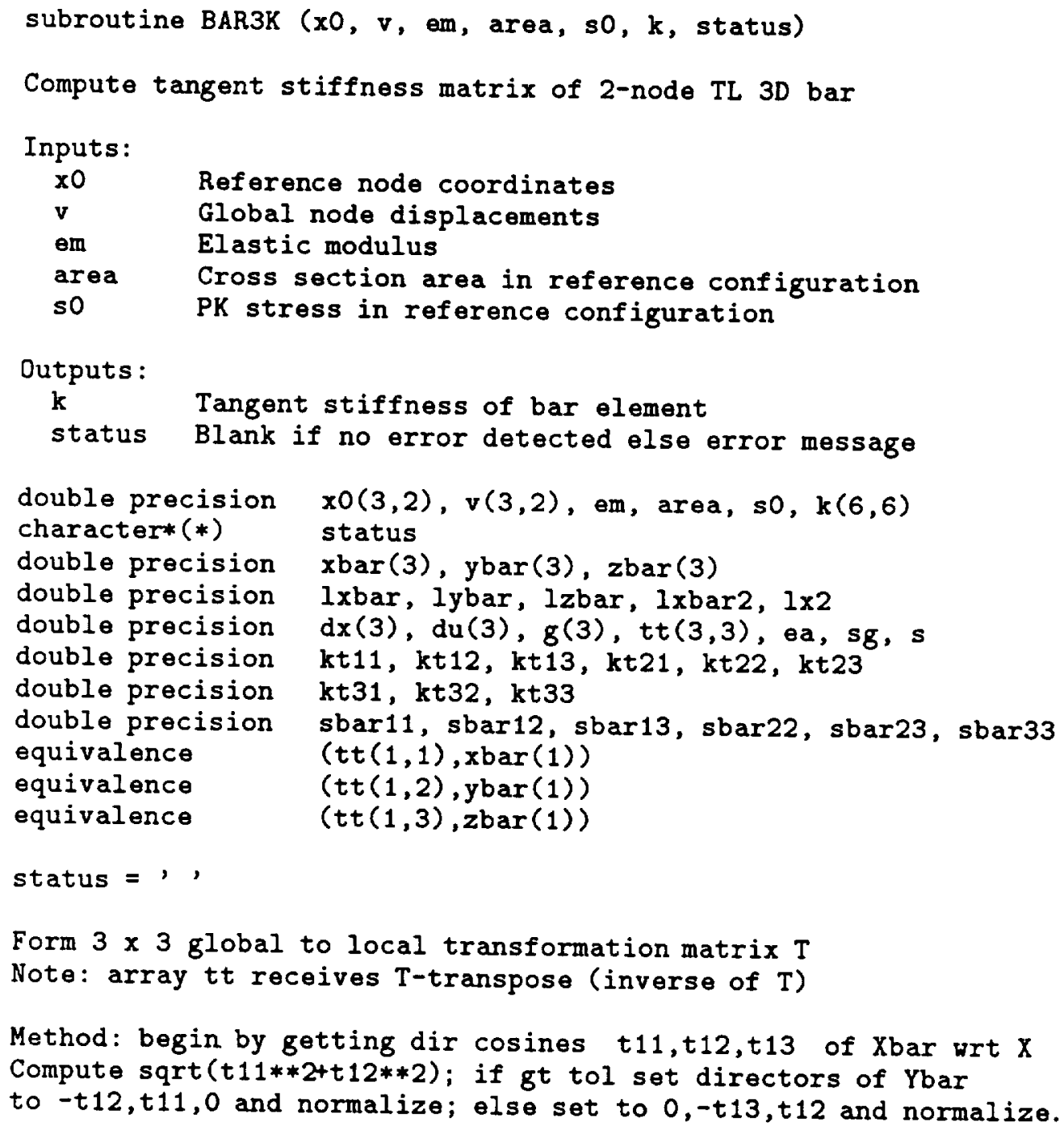


C Value of tol set to .01 but any value ge 0 would do fairly well.

C Finally Zbar $=$ Xbar $\times$ Ybar.

C

$d x(1)=x 0(1,2)-x 0(1,1)$

$d x(2)=x 0(2,2)-x 0(2,1)$

$\mathrm{dx}(3)=x 0(3,2)-x 0(3,1)$

$1 \mathrm{xbar} 2=\mathrm{dx}(1) * * 2+\mathrm{dx}(2) * * 2+\mathrm{dx}(3) * * 2$

1 xbar $=\operatorname{sqrt}(1 \times b a r 2)$

if (lxbar .eq. 0.0) then

status = 'BAR3K: Bar has zero length'

return

end if

$x \operatorname{bar}(1)=\mathrm{dx}(1) / 1 \mathrm{xbar}$

$x \operatorname{bar}(2)=d x(2) / 1 \times$ bar

xbar $(3)=d x(3) / 1$ xbar

1 ybar $=\operatorname{sqrt}(x b a r(1) * * 2+x \operatorname{bar}(2) * * 2)$

if (lybar.gt. 0.01) then

$y \operatorname{bar}(1)=-x \operatorname{bar}(2) / 1$ ybar

ybar $(2)=x \operatorname{cbar}(1) /$ lybar

$y \operatorname{bar}(3)=0.0$

else

lybar $=\operatorname{sqrt}(x \operatorname{xar}(2) * * 2+x \operatorname{bar}(3) * * 2)$

$y \operatorname{bar}(2)=-x b a r(3) / 1 y b a r$

ybar(3) $=x \operatorname{xbar}(2) / 1$ ybar

$\operatorname{ybar}(1)=0.0$

end if

$\operatorname{zbar}(1)=x \operatorname{xbar}(2) * \operatorname{ybar}(3)-x \operatorname{bar}(3) * \operatorname{ybar}(2)$

$\operatorname{zbar}(2)=x \operatorname{bar}(3) * \operatorname{ybar}(1)-x \operatorname{bar}(1) * \operatorname{ybar}(3)$

$\operatorname{zbar}(3)=x \operatorname{xbar}(1) * \operatorname{ybar}(2)-x \operatorname{bar}(2) * \operatorname{ybar}(1)$

lzbar $=\operatorname{sqrt}(\mathrm{zbar}(1) * * 2+\operatorname{zbar}(2) * * 2+\operatorname{zbar}(3) * * 2)$

if (lzbar.ne. 1.0) then

status = 'BAR3K: lzbar ne 1.0: cannot happen'

return

end if

Form core material and geometric stiffness in local coordinates Note: only six different entries of sbar need to be computed

because

$$
\text { Kbar }=\begin{array}{rr}
R & -R \\
-R & R
\end{array}
$$

where

$$
R=\quad \begin{array}{lll}
\text { sbar11 } & \text { sbar12 } & \text { sbar13 } \\
& \text { sbar22 } & \text { sbar23 } \\
\text { symm } & & \text { sbar33 }
\end{array}
$$

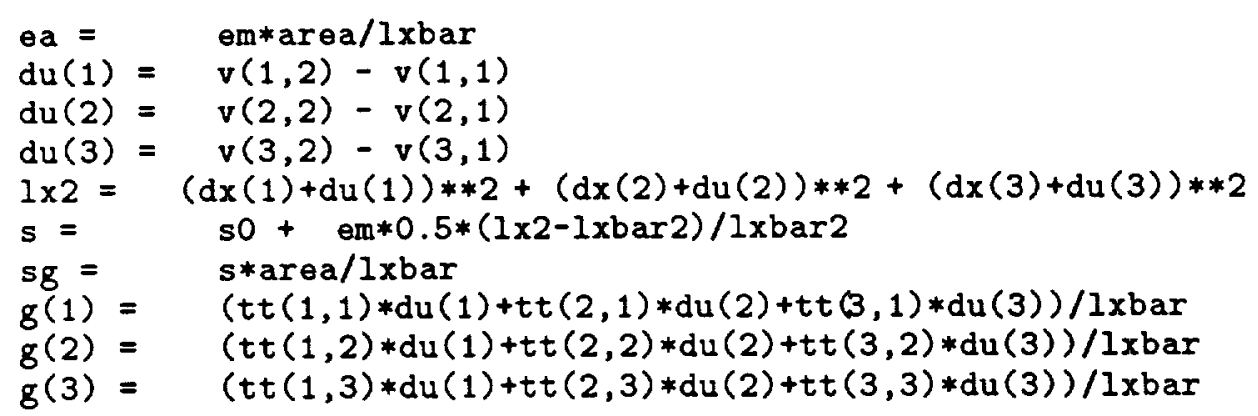




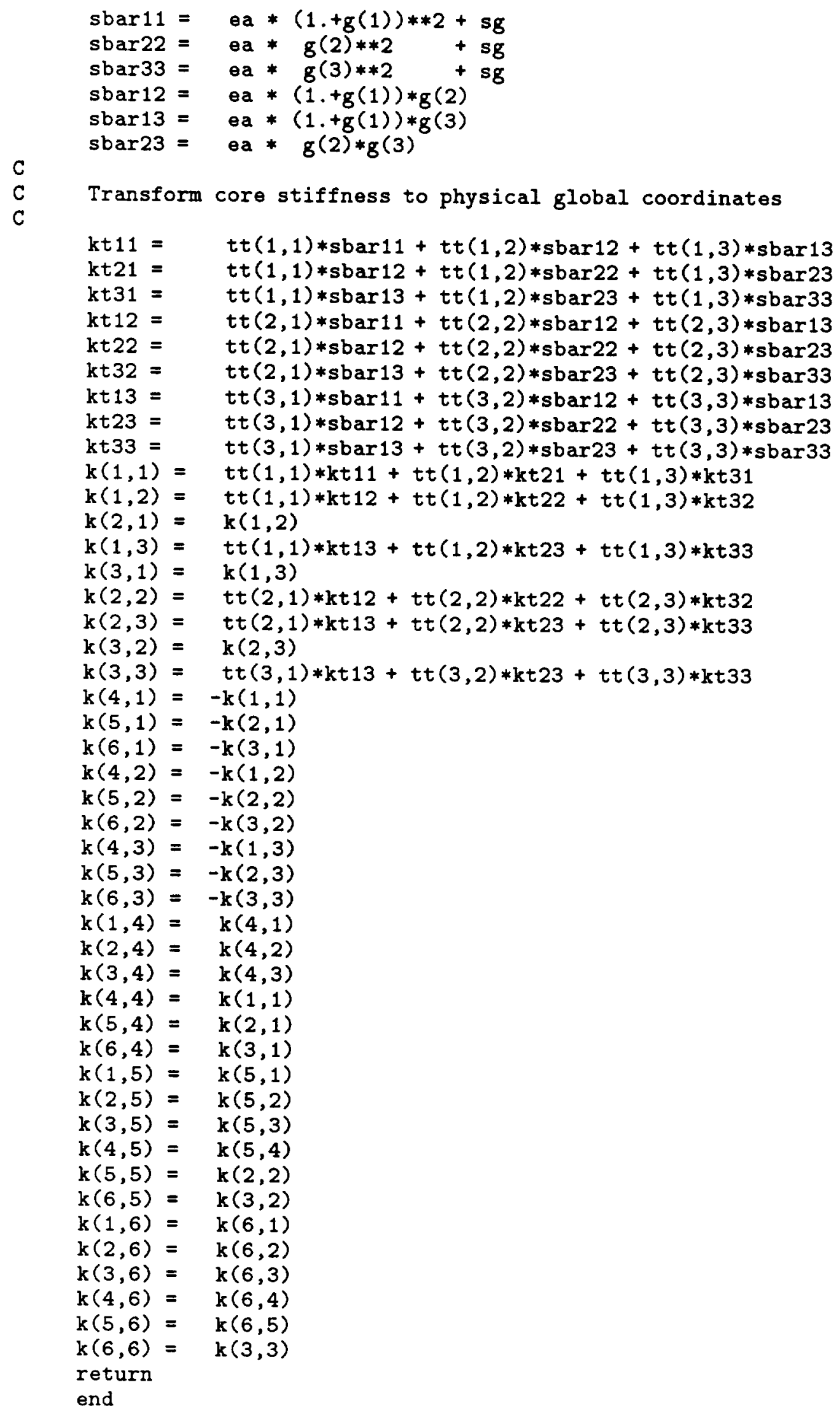


$-$

-

$-$

n.

-

-

-

-

-

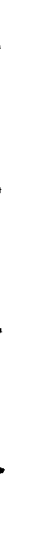

